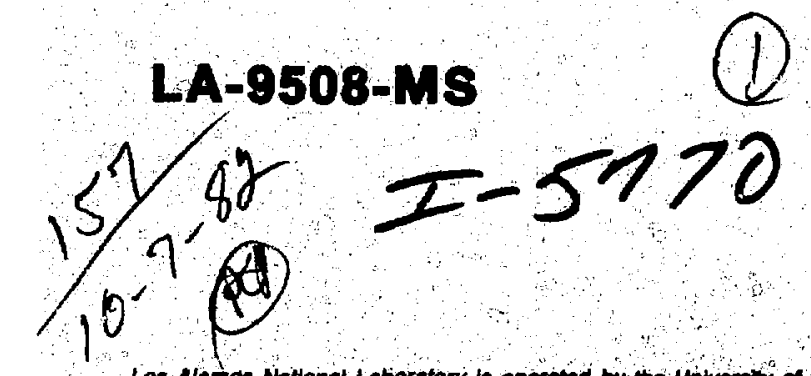

$d r 890$

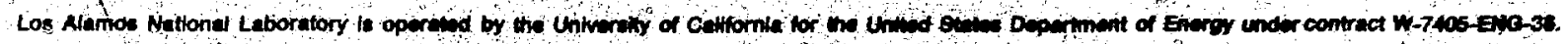

s

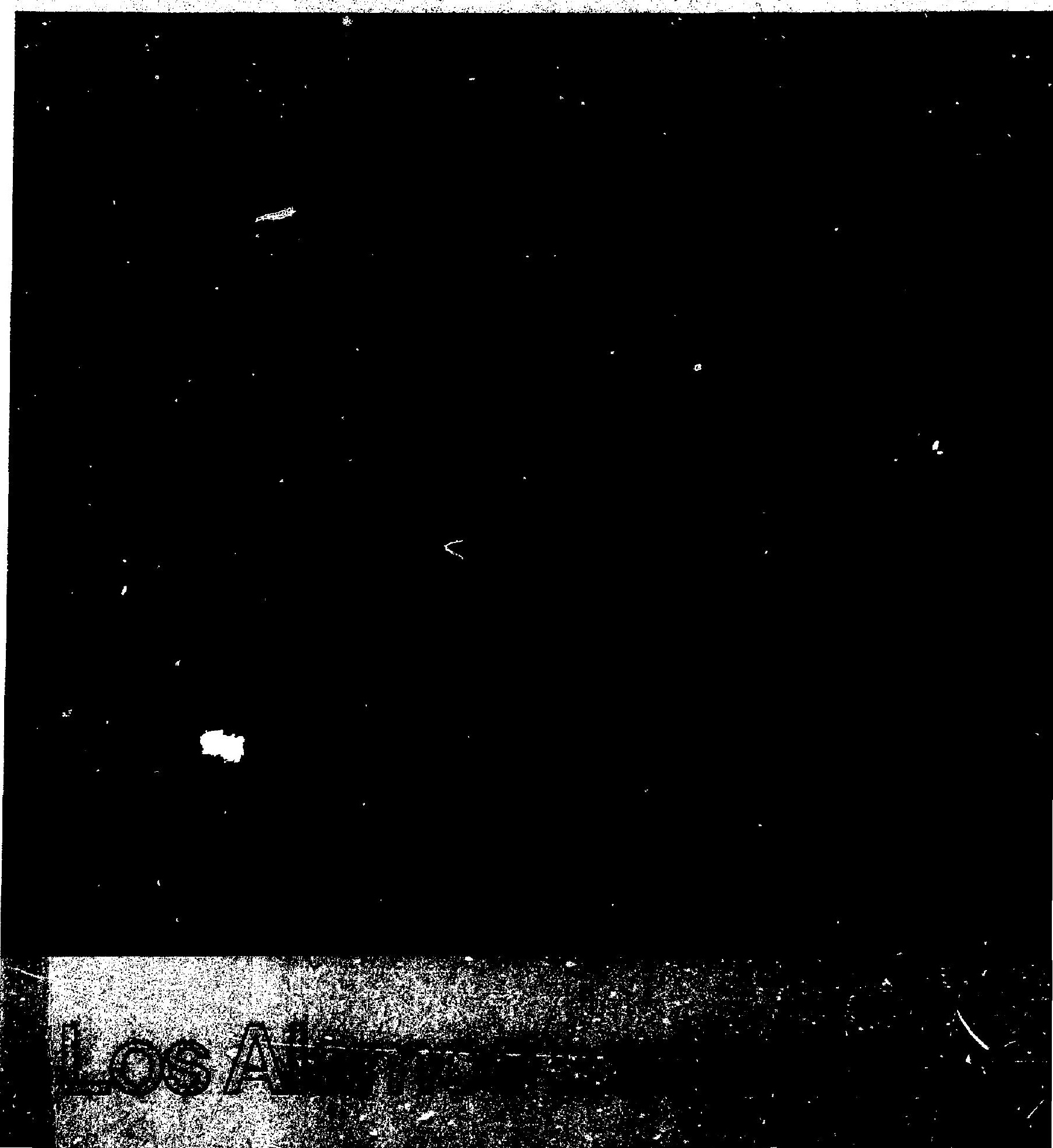


$L A--9508-M S$

DE83 000473

\section{LA-9508-MS}

UC-48

Issued: August 1982

\title{
High-Intensity X-Ray Holography: An Approach to High-Resolution Snapshot Imaging of Biological Specimens
}

\author{
Johndale C. Solem
}

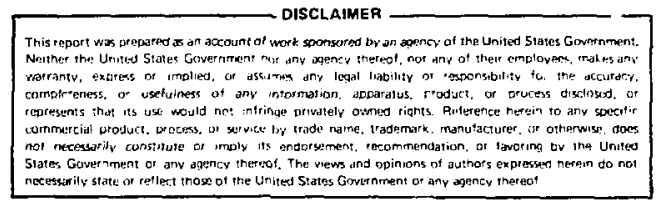


CONTENTS

ABSTRACT . . . . . . . . . . . . . . . . 1

I. INTRODUCTION . . . . . . . . . . . . . . . 2

A. Prospects and Motivation ............. 2

B. History .. . . . . . . . . . . . . . . . 4

C. Scope and Organization . . . . . . . . . . 6

II. CONSIDERATIONS IN SELECTING WAVELENGTH . . . . . . . . 6

III. HOLOGRAPHIC TECHNIQUES . . . . . . . . . . . . . . 7

A. On-Axis Fresnel Transform (Gabor Holography) . . . 9

B. Off-Axis Fresnel Transform

(Leith-Upatnieks Holography) . . . . . . . . . . . 12

C. Planar Fourier Transform (Stroke Holography) . . . 13

D. Spherical Fourier Transform (Skrinsky Holography) . 15

IV. RECORDING METHODS FOR FRESNEL TRANSFORM HOLOGRAPHY . . . 16

A. Photoresist Recording . . . . . . . . . . 16

B. Electron Microscope Recording.......... . 17

C. Shadow Foils .. . . . . . . . . . . . 19

V. REALIZATION OF FOURIER TRANSFORM HOLOGRAPHY AT X-RAY WAVELENGTHS . . . . . . . . . . . . . . 19

A. Planar Fourier Transform ........... 19

B. Spherical Fourier Transform ... . . . . . . . 19

C. Recording Media for Fourier Transform . . . . . . 21

VI. COMPARISON OF HOLOGRAPHIC TECHNIQUES . . . . . . . . 22

VII. HYDRODYNAMIC BLURRING MECHANISMS . . . . . . . . . 22

A. Explosions in a Transparent Medium . . . . . . 23

B. The Effect of Electron Transport . . . . . . . 24

VIII. INTENSITY REQUIREMENTS . . . . . . . . . . . . 25

A. Optimum Exposure Time . . . . . . . . . . 26

B. Intensity . . . . . . . . . . . . . . . 28

C. Meaning of the Cross Sections... . . . . . . 29

D. The Intermediate Case . . . . . . . . . . . . 32

IX. INTENSITY AND PULSE LENGTH CALCULATIONS . . . . . . . . 33

A. Short Pulse Holography .............. 33

B. Long Pulse Holography . . . . . . . . . . . . . 36

C. Holography with an Initially Hot Specinen . . . . 38 
X. THE EFFECT OF COHERENCE ON RESOLUTION .......... 41

A. On-Axis Fresnel Transform............. 41

B. Off-Axis Fresnel Transform . . . . . . . . . . 43

C. Planar Fourier Transform . . . . . . . . . . . . . 44

D. Spherical Fourier Transform . . . . . . . . . . 45

XI. DOPPLER BROADENING . . . . . . . . . . . . . . . . 46

XII. COMPARISON OF COHERENCE REQUIREMENTS . . . . . . . . . 48

XIII. CONCLUSIONS . . . . . . . . . . . . . . . 49

APPENDIX A: CONSIDERATION IN SELECTING SPECIMENS

AND WAVELENGTH . . . . . . . . . . . . . . 51

I. SPECIMEN TYPES ............... . 51

II. STANDARD COMPOSITIONS FOR BIOLOGICAL CONSTITUENTS . . . . . . . . . . . . . 52

III. THE SPECIAL PROPERTIES OF NITROGEN . . . . . . 55

IV. CONTRAST RATIO FOR BIOLOGICAL CONSTITUENTS . . 56

V. ELIGIBILITY OF VARIOUS BIOLOGICAL SPECIMENS FOR X-RAY HOLOGRAPHY . . . . . . 57

APPENDIX B: GRAINSIZE INDEPENDENT HOLOGRAPHY . . . . . 60

I. PLANAR FOURIER TRANSFORM HOLOGRAPHY .... . 60

II. SPHERICAL FOURIER TRANSFORM HOLOGRAPHY . . . . 62

III. THEOREM ON RESOLUTION OF FOURIER TRANSFORM HOLOGRAPHY USING A FRESNEL ZONE PLATE TO GENERATE THE REFERENCE SOURCE. . . . . . . 63

APPENDIX C: IMAGE BLURRING BY A LENSLESS ELECTRON

MICROSCOPE . . . . . . . . . . . . . . . . 64

APPENDIX D: EXPLOSION OF LASER IRRADIATED PROTEIN GLOBULES IMBEDDED IN A TRANSPARENT CYTOPLASM . . . 67

I. DEPOSITION OF LASER ENERGY . . . . . . . . 67

II. EQUATIONS FOR EXPLODING PROTEIN GLOBULE • • 68

III. EARLY-TIME SOLUTION . . . . . . . . . . . 71

IV. LATE-TIME SOLUTION . . . . . . . . . . . 74

V. COMPARISON OF ANALYTIC FORMULAE WITH NUMERICAL CALCULATIONS ........... . 76

VI. FREE-RUN EXPANSIONS ............ 79

APPENDIX E: THE EFFECT OF COHERENCE AND SPECIMEN VOLUME ON RESOLUTION . . . . . . . . . . . . . 82

I. ON-AXIS FRESNEL TRANSFORM . . . . . . . . 82

A. Temporal Coherence Length . . . . . . 82

B. Spatial Coherence Length . . . . . . 84

C. Maximun Volume of Specinen ...... 85 
II. OFF-AXIS FRESNEL TRANSFORM . . . . . . . . 86
A. Temporal Coherence Length . . . . . . 86
B. Spatial Coherence Length . . . . . . . 89
C. Maximum Volume of Specimen . . . . . . 90

III. PLANAR FOURIER TRANSFORM . . . . . . . . . 90
A. Temporal Coherence Length... . . . . 90
B. Spatial Coherence Length . . . . . . . 91
C. Maximum Volume of Specimen . . . . . . 91

IV. SPHERICAL FOURIER TRANSFORM . . . . . . . 92

A. Temporal Coherence Length ....... . 92

B. Spatial Coherence Length . . . . . . . . 92

C. Maximum Volume of Specimen ...... . 93

APPENDIX F: EFFECT OF DOPPLER BROADENING ON

RESOLUTION . . . . . . . . . . . . . . . . . . 94

I. MAXIMUM DISTANCE FROM SPECIMEN TO RECORDING SURFACE . . . . . . . . . . . . . 94

II. MAXIMUM VOLUME OF SPECIMEN . . . . . . . . . . 97

III. DOPPLER BROADENING IN SEMIOPAQUE SPECIMENS . 98

IV. DOPPLER BROADENING FOR OTHER HOLOGRAPHIC TECHNIQUES . . . . . . . . . . . . . . . . . 99

APPENDIX G: THE EFFECT OF HEAT TRANSPORT ON HYDRODYNAMIC BLURRING . . . . . . . . . . . . . . 100

I. RADIATION TRANSPORT ............ 100

II. THERMAL CONDUCTIVITY . . . . . . . . . . . . 101

III. FAST ELECTRON TRANSPORT . . . . . . . . 102

A. Early Time ............ . 102

B. Late Time... . . . . . . . . . . 103

APPENDIX H: GLOSSARY OF SYMBOLS . . . . . . . . . 110

I. LOWER CASE ENGLISH . . . . . . . . . . . . . 110

II. UPPER CASE ENGLISH . . . . . . . . . . . . . . 111

III. LOWER CASE GREEK . . . . . . . . . . . . . . . 112

IV. UPPER CASE GREEK . . . . . . . . . . . . . . . 114

ACKNOWLEDGEMENTS . . . . . . . . . . . . . . . 114

REFERENCES ...................... 115 
FIGURES

Fig. 1. Four basic geometries for holography. Both on-axis Fresnel transform (a) and off-axis Fresnel transform (b) use planar reference waves. Planar Fourier transform (c) and spherical Fourier transform (d) use spherical reference waves . . . . . . 10

Fig. 2. On-axis Fresnel transform holography: (a) typical setup of holographic apparatus; (b) diagram demonstrating that Fresnel transform holography is limited by resolution of recording medium ..................... 11

Fig. 3. Off-axis Fresnel transform holography in a configuration that minimizes temporal and spatial coherence requirements . . 13

Fig. 4. Planar Fourier transform holography: (a) idealized configuration of apparatus; (b) simplified arrangement for calculation consisting of reference source and two object scatterers.... . . . . . . . . . . . . . . 14

Fig. 5. Geometry of spherical Fourier transform holography on a sphere of unit radius................... 15

Fig. 6. Conceptual lensless electron microscope with Fraunhofer holograph .. . . . . . . . . . . . . . . 18

Fig. 7. Typical setup for planar Fourier transform holography . . . . 20

Fig. 8. Spherical Fourier transform holography with a parabolic reflector . . . . . . . . . . . . . . . . 20

Fig. 9. Cross sections for interaction of photons with atoms of four biologically important elements, in the 0.1-10 nm region of wavelength. Legend: "PHOTO", photoelectric absorption; "COH", coherent or elastic scattering; "INC", incoherent or inelastic scattering; " $\lambda 2 / 2 \pi$ ", the maximum possible cross section for a resonant interaction . . . . . . . 30

Fig. A-1. K-shell absorption spectrum of $\mathrm{N}_{2}$ as determined photoelectrically by Bianconi et al. Notice enormous resonance at $401.3 \mathrm{eV}$ for the $1 \mathrm{~S} \rightarrow \pi 2 \mathrm{P}$ transition. (Adapted from figure in Ref. 51). . . . . . . . . . . 56

Fig. D-1. Comparison of shockwave radius calculated by a standard Lagrangian technique with analytic formulae for the early-time expansion [Eq. (C-24)] and the late-time expansion [Eq. (C-33)]. Solid lines are the numerical calculations, dashed lines are the late-time analytic formula and dash-dot-dash lines are the early-time analytic formula. Fig. $D-1$ (a) is for $\gamma=1.1$, Fig. $D-1(b)$ is for $\gamma=4 / 3$, and Fig. $D-1(c)$ is for $\gamma=5 / 3$... 
Fig. D-2. Comparison of protein-cytoplasm interface radius calculated by a standard Lagrangian technique with analytic formulae for the early-time expansion [Eq. $(\mathrm{C}-25)]$ and the late-time expansion [Eq. (C-34)]. Solid lines are the numerical calculations, dashed lines are the late-time analytic formula and dashdot-dash lines are the early-time analytic formula. Fig. $D-2(a)$ is for $\gamma=1.1, F i g . D-2(b)$ is for $\gamma=4 / 3$, and Fig. D-2(c) is for $\gamma=5 / 3$..........

Fig. D-3. Comparison of the hydrodynamic doubling time (the time required for a laser heated sphere to double in radius) from the analytic formula with one-dimensional numerical calculations using a standard Lagrangian technique. Dashed line from upper left to middle right is the analytic formula at constant radius of $100 \AA$ as a function of intensity, and circles are points from the numerical calculations. Dashed lines from upper right to lower left are the analytic formula at constant intensities of $10^{8}$ and $10^{10} \mathrm{~W} \cdot \mathrm{cm}^{-2}$ as a function of radius, and crosses are points from the numerical calculations. . . . . . . . . . . . . . . . . 80

Fig. E-1. Pathlength difference that results in degradation of resolution with reduction in coherence (wavepacket) length. Figure E-1(a) shows actual geometry with: $a=$ distance traveled by diffracted or conherently scattered wavepacket. Figure E-1(b) shows overlap (d) of diffracted and reference wavepacket. Overlapping sections produce an interpretable signal while nonoverlapping sections produce noise . . . . . . . . . .

Fig. E-2. Geometry for calculating coherence length requirements of off-axis Fresnel transform holography

Fig. F-1. Vector diagram of scattering of a photon from a moving atom, which results in slight Doppler shift of the photon energy . . . . . . . . . . . . . . . . . . . . 94

Fig. G-1. Numerical calculation of the radius of an exploding sphere of material containing $\mathrm{C}, \mathrm{H}, \mathrm{O}$, and $\mathrm{N}$ imbedded in a medium of the same density and composition. Material has a numerically calculated equation-ofstate and thermal conductivity. Solid line is with conductivity turned on and dashed line with conductivity turned off. . . . . . . . . . . . . . . . . 101

Fig. G-2. Comparison of $\mathrm{g}^{1 / 5}(\gamma)$ with $\hat{G}^{1 / 5}(\gamma)$ as a function of $\gamma$... . 108 
I. COMPOSITION OF TYPICAL BIOLOGICAL SPECIMENS . . . . . . . . 7

II. TOTAL PHOTON CROSS SECTIONS AND ABSORPTION EDGE

ENERGIES OF SOME BIOLOGICALLY INTERESTING ELEMENTS . . . . . 8

III. COMPARISON OF HOLOGRAPHIC TECHNIQUES . . . . . . . . . . 22

IV. ABUNDANCE OF NITROGEN IONIZATION STATES . . . . . . . 39

V. COHERENCE LENGTH NECESSARY TO AVOID LOSS OF RESOLUTION . . . . 42

VI. ON-AXIS FRESNEL TRANSFORM RESOLUTION OBTAINABIE

FOR SMALL PROKARYOTIC CELL . . . . . . . . . . . . . . . 43

VII. ON-AXIS FRESNEL TRANSFORM RESOLUTION OBTAINABLE

FOR TYPICAL EUKARYOTIC CELL . . . . . . . . . . . . . . 44

VIII. COMPARISON OF COHERENCE AND VOLUME REQUIREMENTS . . . . . . . 49

A-I. ELEMENTARY COMPOSITION OF PROTEIN . . . . . . . . . 52

A-II. ELEMENTARY COMPOSITION OF THE NUCLEIC ACIDS . . . . . . . 54

A-III. STANDARD COMPOSITIONS OF MOST ABUNDANT

BIOLOGICAL CONSTITUENTS . . . . . . . . . . . . . 54

A-IV. X-RAY ABSORPTION LENGTHS FOR MOST ABUNDANT

BIOLOGICAL CONSTITUENTS . . . . . . . . . . . . . . 57

A-V. MASS, DIMENSIONS, AND X-RAY TRANSMISSION OF

VARIOUS BIOLOGICAL SPECIMENS ............. 58

A-VI. X-RAY TRANSMISSION OF "SQUEEZED" CELLS . . . . . . . . . . . . 59 


\title{
HIGH-INTENSITY X-RAY HOLOGRAPHY : AN APPROACH \\ TO HIGH-RESOLUTION SNAPSHOT IMAGING OF BIOLOGICAL SPECIMENS
}

\author{
by
}

Johndale C. Solem

\begin{abstract}
$I$ address the crutickl physical and technological issues pertaining to the holographic imaging of biological structures with a short-pulse, high-intensity, high-quantumenergy laser. I discuss the limitations of $x$-ray optics; how they can be circumvented; and how we can extract an image from the exploding "plasma ghost" of a biological specimen as it existed in the living state picoseconds before the laser pulse.

I consider alternative holographic techniques and find that far-field Fresnel transform holography (Fraunhofer holography) using a photoresist recording surface is most tractable with near term technology. Although it has the advantage of time gating, using an in-line electron microscope for hologram recording presents a difficult tradeoff between quantum efficiency and resolution. Fourier transform holography using a reflector to generate the reference beam and recording with an $x$-ray photocathode and gated microchannelplate might be an attractive alternative, but it is necessarily more complicated.

I discuss the hydrodynamic expansion of inhomogeneities within the specimen, show that expansion is the major source of image blurring, and derive expressions for the intensity and pulse length necessary to obtain high contrast images with minimal blurring. To this end, I derive analytic expressions for the explosion of protein concentrations in an $x$-ray transparent cytoplasm, compare these with numerical calculations, and derive corrections to account for the competitive transport processes by which these inhomogeneities lose energy. I discuss the way in which the absorption and scattering cross sections of individual atoms combine to produce a total absorption and scattering cross section for a discernable feature within a biological structure, and how this understanding affects the equations for optimum intensity and pulse length. To obtain $100 \AA$ resolution, I find optimal intensities of $10^{11}$ to $10^{12} \mathrm{~W} \cdot \mathrm{cm}^{-2}$ and optimal pulse lengths of a few picoseconds. Because these are very short
\end{abstract}


pulses, I consider techniques for gating the recording surface and shuttering the laser beam; and I derive expressions for the best resolution obtainable if forced to use long pulses. I treat in quantitative detail the effect of coherence and Doppler broadening on the maximum distance from the recording surface to the specimen and on the maximum volume of the specimen.

I conclude that: (i) for the near term Fresnel transform holography, particularly, far-field or Fraunhofer holography, seems more practical than Fouricr transform holography; (2) of the alternative fine grain recording media for use with Fresnel transform holography, a photoresist is most attractive; (3) for best resolution, exposure times must be limited to a few picoseconds, and this calls for investigation of mechanisms to shutter the laser or gate the recording surface; (4) the best contrast ratio between the nitrogen-bearing polymers (protein and the nucleic acids) and water is between the K-edges of oxygen and nitrogen; (5) imaging the distribution of nitrogen within a cell, as opposed to other elements, is iikely to be most scientifically rewarding in the near term; and (6) resolution increases with $x$-ray cross section, and, to get the largest cross section, we will want to choose a laser wavelength to drive a resonant transition of our selected element within the specimen.

\section{INTRODUCTION}

\section{A. Prospects and Motivation}

In this paper I discuss holography of biological specimens with very intense, short-pulse, high-quantum-energy lasers. While the research potential for x-ray holography is far broader in scope, this paper is specialized to the problems that attend imaging of viruses, cells, and organelles within cells. The prospective benefits to the biologist are: (1) high-resolution imaging of living specimens in three dimensions; (2) the ability to distinguish individual atomic speci:: and to produce high contrast among biological constituents without staining; and ( 3 ) the ability to freeze mechanical action within a cell on the time scale of picoseconds.

The ability to make high-resolution pictures of cells is the most obviously attractive feature of $x$-ray holography. While very high resolutions have been obtained with electron microscopes, it is difficult to image a living cell. 
Specimens must normally be fixed in some way, and the process of fixation is frequently blamed for unusual structures observed in these specimens. Best contrasts in electron micrographs are obtained by either freeze drying the specimen and sputtering on some high-z element such as osmium or uranium, or chemically infusing a high-z element to locate in particular sites. Such a process can easily alter the structure within the cell to something differing significantly from the way it was when the cell was alive. ${ }^{1}$ With the highintensity $x$-ray laser, we take a snapshot of the cell. The cell is destroyed in the process, and what we record is the "plasma ghost" of the cell as it existed in the living state a few picoseconds before. The image could be made from living cell cultures, or even cells in living tissue.

The three-dimensional character of holograms is an added benefit. A hologram is not merely stereoscopic, but records complete positional information of structural elements within the specimen to the extent that they are visible within a restricted range of angle. Not only can a magnified view of the specimen be displayed by usual holographic techniques, but planar cross sections can be generated by computer for various angles and depths. Techniques for computer reconstruction of holograms have been in use for 15 years, ${ }^{2}$ but widespread use of these techniques has beer. limited mainly owing to the sparsity of research applications for holographic imaging. Good cross sectional images by electron microscope now not only require killing and fixing the cell, but it also must be cut into thin sections. ${ }^{3}$

The ability to distinguish individual atomic species or even specific molecular bonds is already generally appreciated by biologists. The electron microscope lacks this feature, and the use of fluorescence in optical microscopy has been crucial to certain types of research. ${ }^{4}$ Part of the motivation for $x$-ray microscopes using synchrotron radiation has been the ability to distinguish atomic species. ${ }^{5}$ Although $x$-ray lasers with continuously tunable wavelength are unlikely to be available soon, it may be quite possible to find lasing transitions that lie close to the K-edges of biologically interesting elements, or may even coincide with specific resonances.

The utility of snapshot imaging is less obvious. We know of few processes within any organism that take place on the microsecond scale, let alone the nanosecond or picosecond scale. Is this because they do not exist or because we have never had the means to look? A few exciting possibilities are apparent. 
We may be able to see structures that have so far been invisible in living cells because of thermal motion. ${ }^{6}$ We may also be able to see changes in molecular conformation such as those that are suspected to be responsible for signal promulgation in sensory ceils. A specific example is the lightdriven change in topology that is suspected to occur in the macromolecules that reside in lipidbilayer disks of retinal cells. ${ }^{7}$ There are numerous other examples, such as being able to see the penetration of vesicles of neurotransmitter through the presynaptic membrane in the process of transfer of a signal from one nerve cell to another. ${ }^{8}$ In particular we might watch the flow of calcium in this process. ${ }^{9}$

The specific features of $x$-ray holography that become most useful to biological science may be beyond speculation at this time because no one knows what will be discovered in the first experiments, nor the tine scale over which the technology will grow. If history is any guide, however, we know that many techniques thought to be impossible a decade ago are routine today.

\section{B. History}

Ever since Gabor's first conception of the principle of reconstructing a wavefront from a photograph of the interference fringe-pattern of a scattered and a reference wave 10,11 researchers have speculated whether a source of coherent radiation might be used to make holograms of elementary biochemical structures at an atomic or molecular level of resolution.

In 1952, Baez ${ }^{12}$ derived a necessary relationship among wavelength, system dimensions, and the resolving power of the recording medium for plane wave holography. From it he concluded that holograms can probably be made with $X$ radiation; moreover, he noted that, if a sufficiently fine-grained hologram were recorded with a very minute monochromatic $X$-ray source, then a greatly magnified visible image in three dimensions could be reconstructed with visible illumination. Baez and El-Sum ${ }^{13}$ extended this analysis to include the effects of finite bandwidth, penetration of the specimen, and film grain size.

Stroke and Falconer ${ }^{14}$ suggested that the grain-size limitation could be circumvented by using curved wavefronts for both the reference and the scattered radiation--a technique they called "Fourier-transform holography." Stroke and Restrick ${ }^{15}$ obtained visible-light holograms of extended objects with spatially noncoherent radiation, and asserted that only temporal coherence is essential. The physical reason for this is discussed later in this paper. Stroke ${ }^{16}$ specifically considered the problem of high-resolution X-ray holography and noted 
that with the Fourier technique even Mössbauer gamma radiation might be used for holography. Rogers and Palmer ${ }^{17}$ suggested that the focussing properties of zone plates might facilitate Fourier transform holography with non-laser sources of $X$ rays.

In 1970, however, Breedlove and Trammel1 18 asserted that a much more fundamental difficulty makes atomic-scale holography impossible. Coherent scattering cross sections are far less than the corresponding photoelectric absorption cross sections; therefore, they asserted, molecules would be destroyed by ionization before enough photons could be scattered from each atom to form the hologram. I shall discuss the fallacy of this assertion later.

Interest in longer-wavelength holography continued, however. Aoki et al. made holograms with carbon $\mathrm{K}$-alpha $(44.8 \AA),{ }^{19}$ electron synchrotron $(60 \AA)$, and aluminum $\mathrm{K}$-alpha $(8.3 \AA)^{20}$ radiations and reconstructed then with light from a helium-neon laser. The lateral resolutions achieved, of the order of $4 \mu \mathrm{m}$, were comparable with the theoretical limit found by Baez and El-Sum, ${ }^{13}$ although longitudinal resolutions were much poorer. Reuter and $\mathrm{Mahr}^{21}$ also made holograms at $44.8 \AA$ with somewhat poorer resolution.

Bjorklund 22,23 described a method of making grainless holograms, using a polymer which was made developable in an etching agent by exposure to far ultraviolet radiation. He speculated on the possibility of X-ray holography of molecules, apparently unaware of the negative pronouncement of Breedlove and Trammell, and he briefly suggested a recording apparatus for this purpose.

The negative conclusion of Breedlove and Trammell 18 was challenged by Chapline and Wood. ${ }^{24}$ They asserted that, if the required X-ray fluence could be delivered before the molecule had dissociated, then a hologram might still be obtained on an atomic scale of resolution.

A general discussion of the intensity requirements for x-ray holography of microobjects has been given by Kondratenko and Skrinsky. 25 In particular, they gave formulae for the required exposure time of a Fourier transform hologram, taking into account quantum noise effects. The intensity requirements for holography at wavelengths near $1 \AA$ has also been discussed by Mueller and Jorna. ${ }^{26}$ However, they restricted their discussion to the case of Fresnel transform holography, and so, their estimates of required intensities are much larger than optimal. For example, to obtain an undistorted image of the plane wave hologram they proposed using a monolayer of photoemissive material, which is limited to a quantum efficiency less than $10^{-4}$. On the other hand, for the 
case of Fourier holography, one could use detectors with a quantum efficiency near unity, as Kondratenko and Skrinsky ${ }^{25}$ point out.

\section{Scope and Organization}

This paper combines calculations from a broad range of discip.Lines necessary to estimate the resolution of holograms of microbiological specimens produced with a high-intensity $x$-ray laser. Among the problems that must be considered are: (1) selection of specimen and wavelength, (2) grain-size independent holographic techniques, (3) fine-grain image-recording technologies, (4) mechanisms of hydrodynamic blurring, (5) energy transport processes within the specimen, and (6) the affect of coherence and Doppler broadening. Rather extensive original technical work is presented, bearing on the central issues. Because of the length of the derivations and calculations, I have divided the paper into a main text that discusses the impact of the central issues, and series of appendices that cover each technical point in detail.

\section{CONSIDERATIONS IN SELECTING WAVELENGTH}

Table I shows the composition of typical active eukaryotic ${ }^{27}$ cells, prokaryotic cells, 28,29 and viruses. ${ }^{28}$ A eukaryote is a cell having well defined nucleus, such as those found in higher plants and animals. A prokaryotic cell has no nucleus and is almost always a one-celled organism such as a bacterium. It differs in composition from the eukaryote most conspicucusly in its relative content of ribonucleic acid (RNA) and deoxyribonucleic acid (DNA). For example, the bacterium Escherichia coli (E. coli) contains approximately $6 \%$ RNA and $1 \%$ DNA. Viruses consist of nucleic acid attached to special proteins and, at most, only traces of the other constituents. The lipids are fatty materials containing mostly carbon, hydrogen, and oxygen. The other organic materials are carbohydrates or sugars of various sorts. Carbohydrates are also prominent in structural material of eukaryotic plant cells. The inorganic constituents are mostly salts, containing, in order of importance, potassium, magnesium, sodium, and calcium as cations. DNA and RNA are marked by the presence of phosphorous, but phosphate is also the most abundant anion. Adenosine triphosphate, the energy currency of a cell, and the messenger material adenosinemonophosphate are probably the most interesting phosphorous bearing materials outside the nucleus. 
TABLE I

COMPOSITION OF TYPICAL BIOLOGICAL SPECIMENS

(BY MASS)

\begin{tabular}{|c|c|c|c|}
\hline Constituent & $\begin{array}{c}\text { Eukaryotic } \\
\text { Cel1 }\end{array}$ & $\begin{array}{c}\text { Prokaryotic } \\
\text { Cel1 }\end{array}$ & Viron \\
\hline Water & $75-85 \%$ & $65-75 \%$ & - \\
\hline Protein & $10-20 \%$ & $10-20 \%$ & $35-95 \%$ \\
\hline $\begin{array}{l}\text { DNA } \\
\text { RNA }\end{array}$ & $\begin{array}{l}\sim 0.4 \% \\
\sim 0.7 \%\end{array}$ & $\begin{array}{l}1-4 \% \\
6-8 \%\end{array}$ & $5-65 \%$ \\
\hline Iipid & $\sim 2 \%$ & $\sim 6 \%$ & - \\
\hline Carbohydrate & $\sim 1 \%$ & $\sim 3 \%$ & - \\
\hline Salts & $\sim 1 \%$ & $\sim 1 \%$ & - \\
\hline
\end{tabular}

Most of the nitrogen is in proteins and nucleic acids. Of the other nitrogen bearing materials such as urea $\left[\mathrm{CO}\left(\mathrm{NH}_{2}\right)_{2}\right]$ are present in various degrees depending on the cell type.

Some of the elements whose density in biological specimens might be interesting to image are given in Table II along with the energy of their K-edges. We would like to tune the laser near the K-edge, not only because the photoelectric effect is at a local maximum, but resonances, such as those that might involve elevating a $\mathrm{K}$-shell electron to a molecular orbital, will be found in this region. Such resonances will have very large cross sections. Although carbohydrates comprise some of the structural elements of plant cells, in general, an image of the distribution of carbon in a cell is unlikely to be very imformative. 0xygen is even less likely to be informative because of the large content of water. Phosphorus would be interesting because it could be used to image the chromatin, although it might be somewhat obscured by the abundance of phosphate in the protoplasm. Sulfur and calcium also play interesting roles that might be profitably explored with $x$-ray holography. The abundances of phosphorous, sulfur, and calcium are relatively low, and their K-edges are in the 2-3 keV range, which would be more stressing to prospective laser technologies. 
TABLE II

TOTAL PHOTON CROSS SECTIONS AND ABSORPTION EDGE ENERGIES OF SOME BIOLOGICALLY INTERESTING ELEMENTS

\begin{tabular}{|c|c|c|c|c|c|c|}
\hline \multirow[b]{2}{*}{ Element } & \multicolumn{2}{|c|}{ Absorption Edge Energies (keV) } & \multicolumn{4}{|c|}{ Total Photon Cross Section $\left(\mathrm{cm}^{2}\right)$} \\
\hline & $\mathbf{K}$ & $\mathrm{L}$ & $200 \mathrm{eV}$ & $400 \mathrm{eV}$ & $800 \mathrm{eV}$ & $1600 \mathrm{eV}$ \\
\hline Hydrogen & - & - & $2.48 \times 10^{-21}$ & $3.10 \times 10^{-22}$ & $3.88 \times 10^{-23}$ & $4.85 \times 10^{-24}$ \\
\hline Carbon & 0.284 & - & $1.06 \times 10^{-19}$ & $5.22 \times 10^{-14}$ & $8.08 \times 10^{-20}$ & $1.13 \times 10^{-20}$ \\
\hline Nitrogen & 0.400 & - & $1.97 \times 10^{-19}$ & $3.69 \times 10^{-20}$ & $1.41 \times 10^{-19}$ & $2.07 \times 10^{-20}$ \\
\hline Oxygen & 0.532 & - & $3.49 \times 10^{-19}$ & $6.31 \times 10^{-20}$ & $2.23 \times 10^{-19}$ & $3.43 \times 10^{-20}$ \\
\hline Phosphorous & 2.142 & $0.153,0.128$ & $4.26 \times 10^{-18}$ & $9.65 \times 10^{-19}$ & $1.72 \times 10^{-19}$ & $2.71 \times 10^{-20}$ \\
\hline Sulfur & 2.471 & $0.193,0.163,0.162$ & $4.41 \times 10^{-18}$ & $1.27 \times 10^{-18}$ & $2.27 \times 10^{-19}$ & $3.58 \times 10^{-20}$ \\
\hline Calcium & 4.038 & $0.399,0.350,0.346$ & $8.31 \times 10^{-19}$ & $2.63 \times 10^{-18}$ & $5.74 \times 10^{-19}$ & $9.54 \times 10^{-20}$ \\
\hline
\end{tabular}

I believe that nitrogen is the most interesting element to image in initial experiments on the basis of (1) the energy of its K-edge, (2) its relative high abundance, (3) its concentration in the proteins, which tend to be most dense in the structural elements of a cell, and (4) the possible existence of resonances near the nitrogen $\mathrm{K}$-edge in the nucleic acids and proteins, which will greatly enhance their contrast with water (see Appendix A). By tuning to nitrogen we would illuminate the protein in cell membranes, in mitochodrian membranes, in the cytoskeleton, and various structural parts of the nucleus, such as the histones on which DNA is wrapped in eukaryotic cells.

In this paper I will look specifically at the problem of imaging the density of nitrogen with the intent of obtaining a snapshot of the distribution of protein. The principles involved will apply equally well to mapping other elemental concentrations. The choice is in part motivated by the considerations given above and is in part arbitrary. Appendix A discusses contrast ratios and transmissions for various biological specimens, with particular attention to the nitrogen resonance. It also discusses the process by which $I$ have established standard compositions for biological constituents to be used in calculations throughout the paper.

\section{HOLOGRAPHIC TECHNIQUES}

I have investigated numerous holographic techniques and have settled on four possibilities for making holograms of microscopic specimens with a high- 
intensity, short-pulse, high-quantum-energy laser. These are: (1) on-axis Fresnel transform holography, also known as Gabor holography or in-line holography; (2) off-axis Fresnel transform holography, also known as Leith-Upatnieks holography; (3) planar Fourier transform holography, sometimes called Stroke holography; and (4) spherical Fourier transform holography, for which I give credit to Skrinsky. The wavefront patterns for these techniques are compared schematically in Fig. 1.

\section{A. On-Axis Eresnel Transform (Gabor Holography)}

Figure 2a shows a typical setup for an on-axis Fresnel transform holograph. The principal advantage of the technique is simplicity. It requires only one laser beam, and no optical elements other than the specimen and the recording surface. The specimen to be holographed is placed in line with the laser beam which provides both the reference beam and the specimen illumination.

One difficulty of on-axis Fresnel transform holography is that it requires a very high cesolution recording medium. A feeling for this can be derived from an argument given by Stroke, ${ }^{10}$ illustrated in Fig. $2 \mathrm{~b}$. If the object consists of a series of point scatterers separated by distance $\delta$, it can be thought of as a diffraction grating. The angle at which the first maximum in the diffraction pattern will ocsur is $\sin ^{-1}(\lambda / \delta)$. This maximum must interfere with the plane waves of the reference beam that are parallel to the recording surface. In order to record the interfecence pattern between the diffracted beam and the reference beam, the spacing between the grains, $\Delta$, must be at least large enough for alternating grains to record the maxima and minima of the resulting fringes. Consequently, $\Delta \tan \left(\sin ^{-1}(\lambda / \delta)\right) \geq \lambda / 2$, or for small angles $\lambda(\Delta / \delta) \geq \lambda / 2$, which implies that $\delta \geq 2 \Delta$. The minimum spacing that can be resolved must be greatar than twice the grain spacing and is independent of wavelength.

At large angles, which occur when the wavelength approaches the size of the grid spacing, the fringe spacing becomes dependent on the wavelength. The first maximum of the diffraction pattern occurs at a very large angle, and this limits the effectiveness of on-axis Fresnel transform holography because the area of reference illumination will be too small to encompass the peak of the diffraction pattern. If $\lambda=\delta$ the diffraction peak will occur at $90^{\circ}$, and the recording medium would have to be infinite in extent to record the first diffraction peak. Also pointed out by Mueller or Jorna, ${ }^{26}$ at large diffraction angles and short 

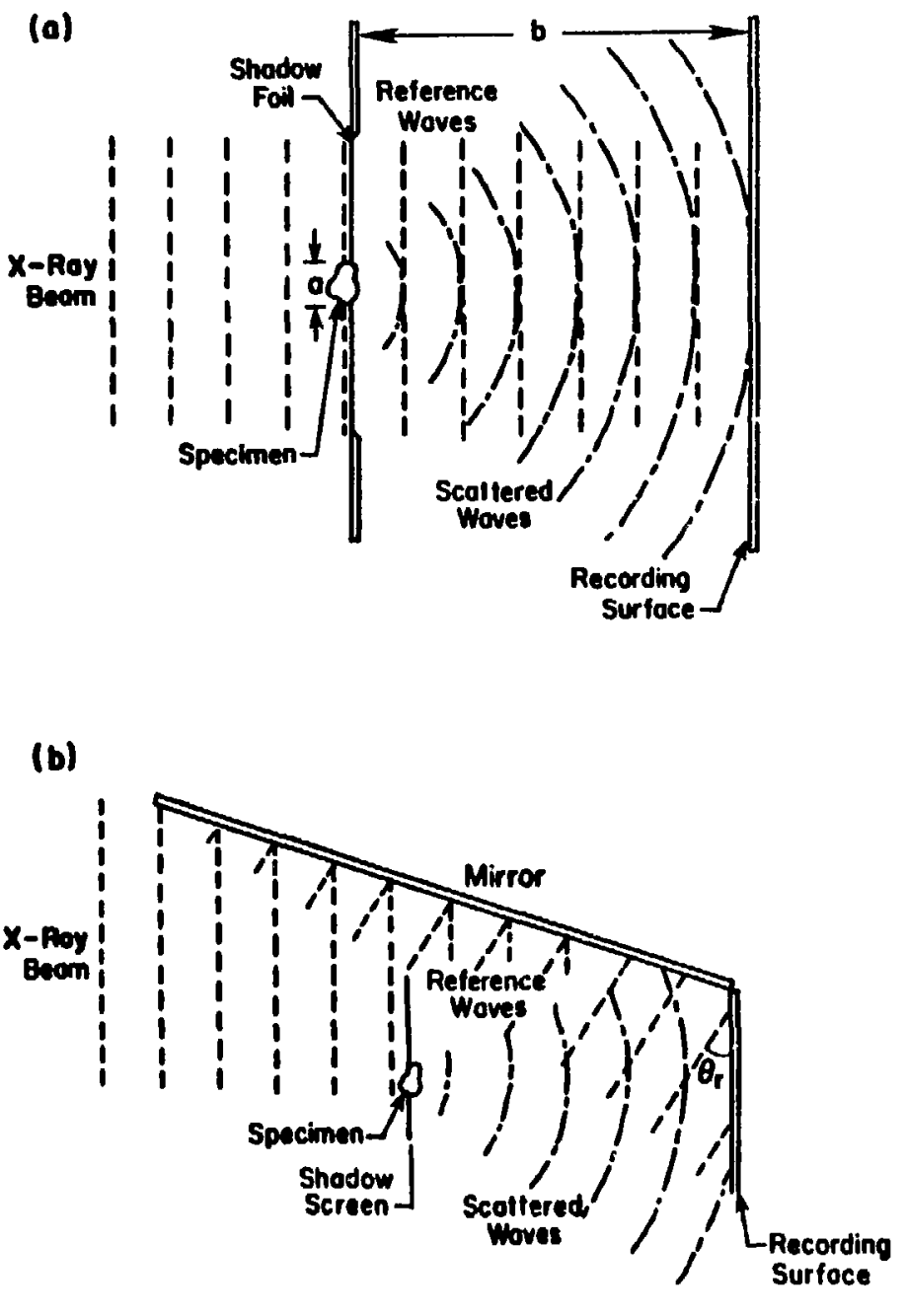

(c)
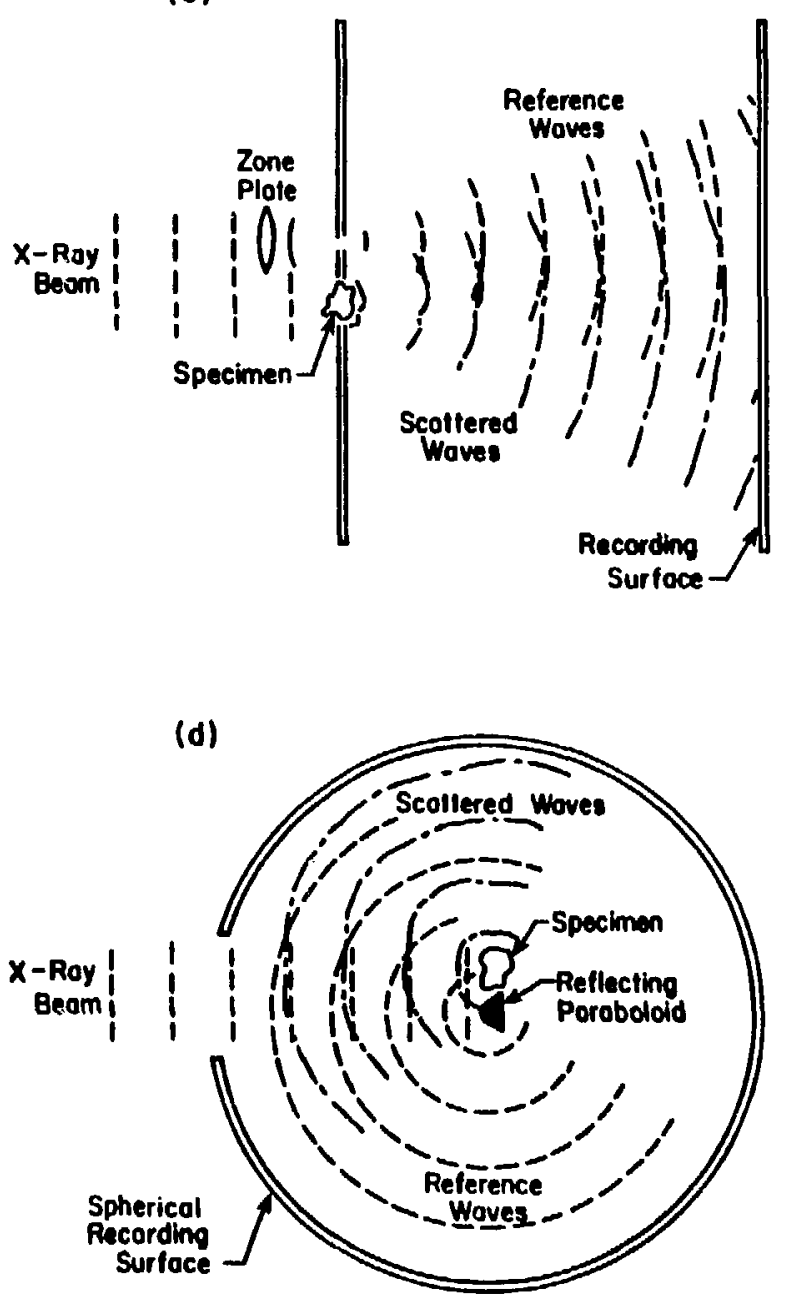

Fig. 1.

Four basic geometries for holography. Both on-axis Fresnel transform (a) and off-axis Fresnel transform (b) use planar reference waves. Planar Fourier transform (c) and spherical Fourier transform (d) use spherical reference waves. 


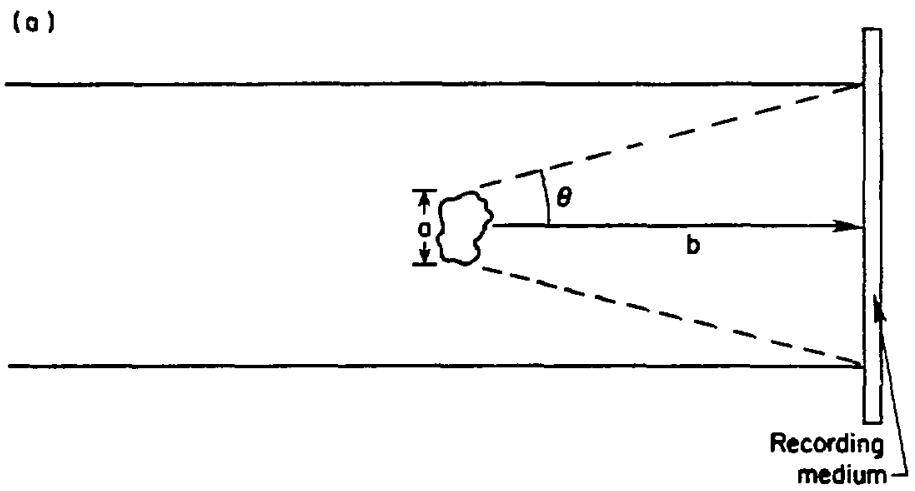

(b)

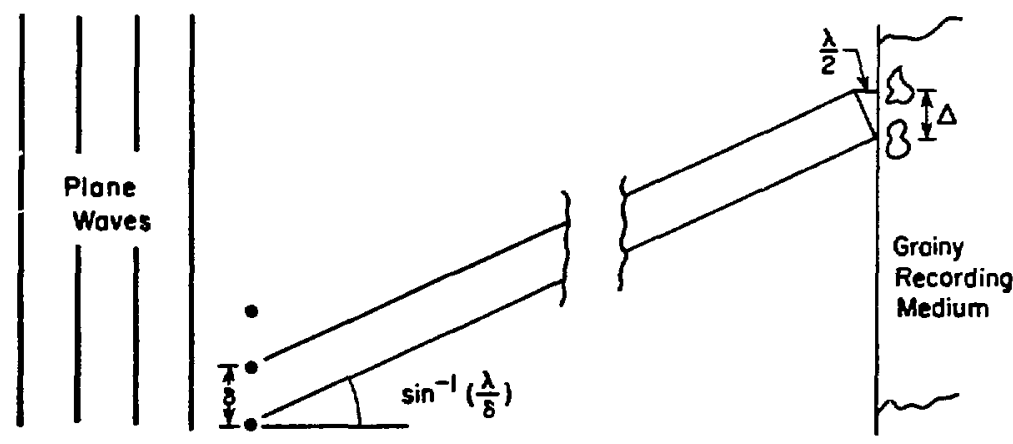

Fig. 2 .

On-axis Fresnel transform holography: (a) typical setup of holographic apparatus; (b) diagram demonstrating that Fresnel transform holography is limited by resolution of recording medium.

wavelengths we would run into difficulty with roughness of the surface of the recording medium.

Another difficulty with on-axis Fresnel transform holography is interference between the real and virtual images in reconstruction. ${ }^{30}$ If the distance between the specimen and the recording surface is large compared to the area of the specimen divided by the wavelength, the superimposed virtual inage is blurred out over the entire reconstruction and results in a low level of background noise that is easily tolerated. Referring to Fig. 2a, the condition is given by 
$b>\frac{a^{2}}{\lambda}$

and this type of recording is frequently called far-field Fraunhofer Holography. If our specimen looks like an array of opaque structures imbedded in a nearly transparent medium, then the area, $a^{2}$, in the above inequality need only be the area subtended by the largest structure. If, on the other hand, the specimen consists of only slightly varying shades of grey, $a^{2}$ is the area of the entire specimen. In the latter case, it may be difficult to achieve the far-field Fraunhofer conditions owing to noise introduced by the coherence-length limitations.

B. Off-Axis Fresnel Transform (Leith-Upatnieks Holography ${ }^{31}$ )

The interference between the real and virtual images in reconstruction can be eliminated by arranging to have the planar reference waves impinge the recording surface at an off-axis angle. This is usually arcomplished by using a mirror as shown in Fig. 3. For complete separation of the virtual and real images we must have

$$
\sin \theta_{\mathrm{r}}>\lambda / \delta
$$

where $\theta_{r}$ is the angle between the planar reference waves and the recording surface, and $\delta$ is the linear resolution we are seeking. Off-axis Fresnel transform holography suffers from the same grain size limitations as the on-axis form and requires an $x$-ray mirror of sufficient precision to retain good spatial coherence in the reference beam. The effective reflecting surface of the mirror must be planar or well-characterized within a fraction of a wavelength over an area on the order of the reference-illuminated area of the recording surface. This is a difficult condition to achieve, especially at high intensity when the characteristics of the mirror may well be functions of time.

It is also interesting to note that if the structure of the object and the wavelength were appropriate for the object to scatter plane waves, then the interference pattern at the recording medium would have a spatial frequency of $(\sin \alpha) / \lambda$ where $\alpha$ is the half-angle of interference. This is true for both onaxis and off-axis forms. 


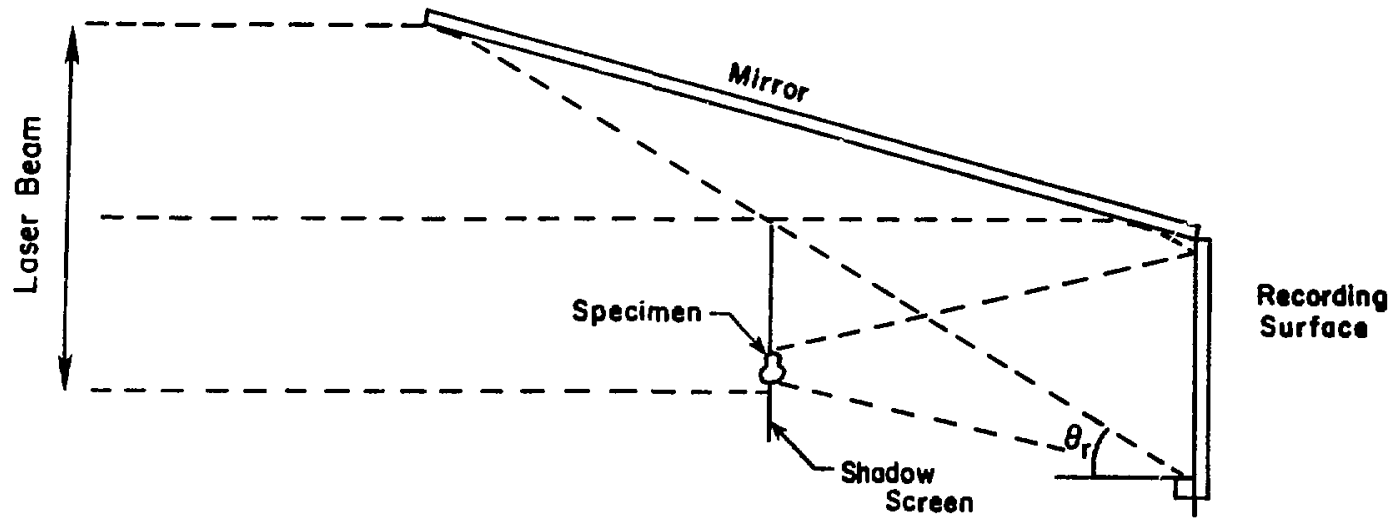

Fig. 3 .

Off-axis Fresnel transform holography in a configuration that minimizes temporal and spatial coherence requirements.

\section{Planar Fourier Transform (Stroke Holography)}

Figure 4a shows an idealized configuration for planar Fourier transform holography. ${ }^{14}$ The reference source emits spherical waves, which interfere with waves from the object at the recording surface. The object is separately illuminated by a plane wave source. Figure $4 b$ shows a simplified arrangement with a reference source, $o$, and two object point scatterers, $x_{1}$ and $x_{2}$, all emitting spherical waves originally at the same phase. The intensity pattern at the screen [see Appendix B] will be given by

$$
I \doteq \frac{A^{2}}{r^{2}}\left[1+\frac{4 a}{A} \cdot \cos \frac{\pi\left(x_{1}+x_{2}\right) \xi}{2 \lambda r} \cdot \cos \frac{\pi\left(x_{1}-x_{2}\right) \xi}{2 \lambda r}\right] \cos \theta
$$

where $\theta$ is the angle between the radius vector, $\vec{r}$, and the plane of the recording surface, $a$ and $A$ are amplitudes of the reference and scattered waves, respectively, and $A \gg$ a. This pattern consists of a dc level plus a rapidly oscillating function of amplitude proportional to the mean distance of the two object scatterers from the reference source modulating a slowly oscillating function of amplitude proportional to the difference in distance between the two object sources. It is called Fourier transform holography because every point on the 

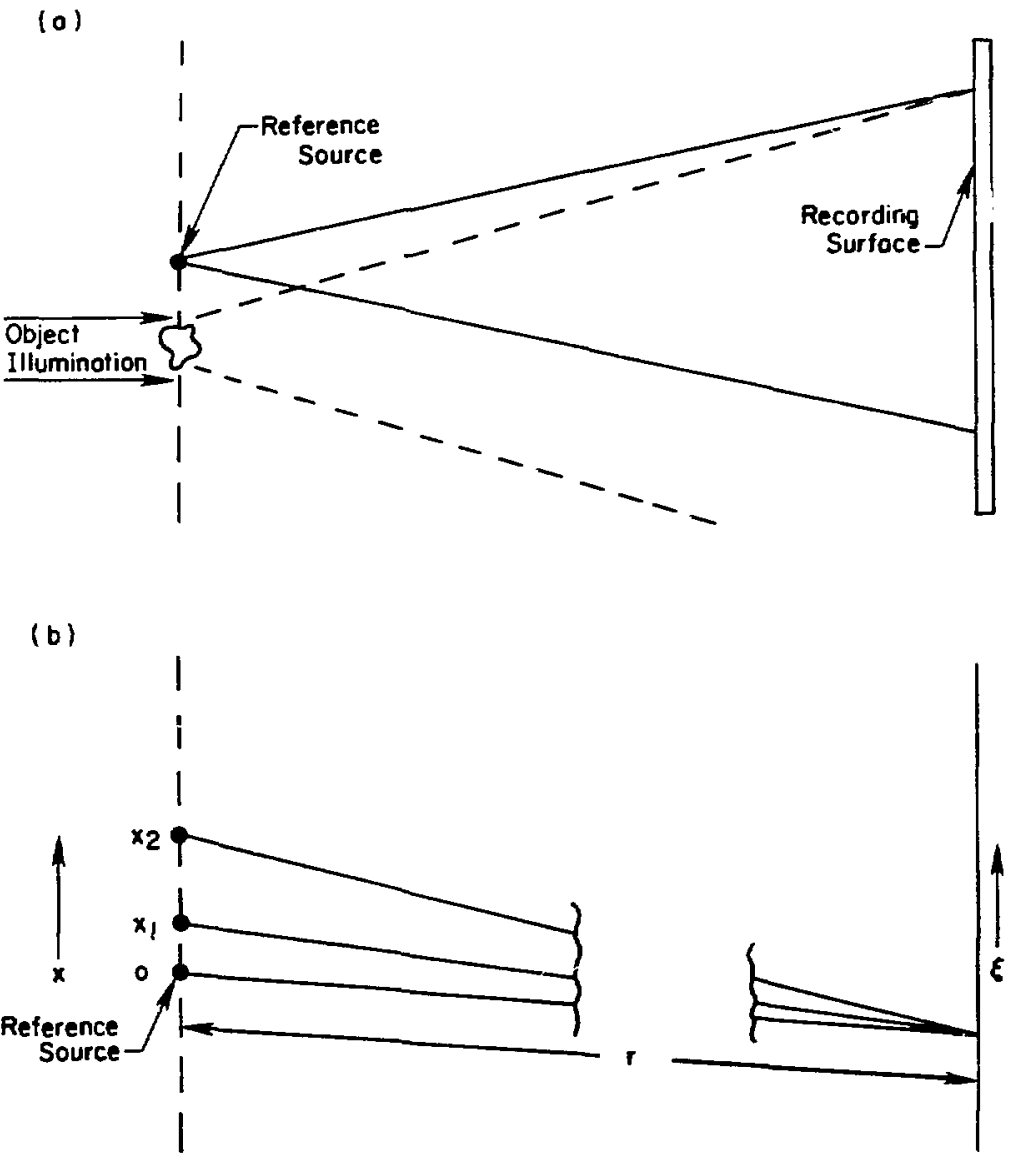

Fig. 4.

Planar Fourier transform holography: (a) idealized configuration of apparatus; (b) simplified arrangement for calculation consisting of reference source and two object scatterers.

reference source maps to unique spatial fringe frequency at the recording surface. It is sometimes called lensless Fourier transform holography because the usual Fourier transforming technique requires a lens to transform spatial information to spatial frequency information. The maximum spatial frequency of the interference pattern can be adjusted arbitrarily by placing the object at various distances from the reference source. Note that difficulty occurs again when the spacing of points in the object is smaller than the wavelength. For example, if $\left(x_{1}-x_{2}\right)<\lambda$, the modulation pattern never reaches a maximum. 
D. Spherical Fourier Transform (Skrinsky Holography)

We can mitigate the large-angle problem by making a spherical recording surface. The geometry on a unit sphere for such a Fourier transform technique is shown in Fig. 5. If we consider the reference source of spherical waves to be at the origin and the object to again consist of two point scatterers at $\mathbf{x}_{1}$ and $x_{2}$, then [see Appendix $B$ ] we find the following intensity pattern:

$$
I \doteq A^{2}\left[1+\frac{4 a}{A} \cdot \cos \frac{\pi\left(x_{1}+x_{2}\right) \sin \theta \cos \phi}{\lambda} \cdot \cos \frac{\pi\left(x_{1}-x_{2}\right) \sin \theta \cos \phi}{\lambda}\right]
$$

where again $A \gg$ a. The intensity pattern consists of bands parallel. to the $y-z$ plane and centered on the $x$-axis. As in the planar case, the spatial (or angular) frequency consists of a rapid oscillatory function proportional to the mean distance of the object points from the reference modulated by a slowly varying function depending on the distance between the object points. Again, if the point spacing is less than the wavelength, one never completes a full cycle. The physical spacing of the fringes can be made arbitrarily large by expanding the radius of the sphere. Thus we could use an ordinary film of arbitrarily large grain size, as long as the tradeoff between sensitivity and resolution was favorable.

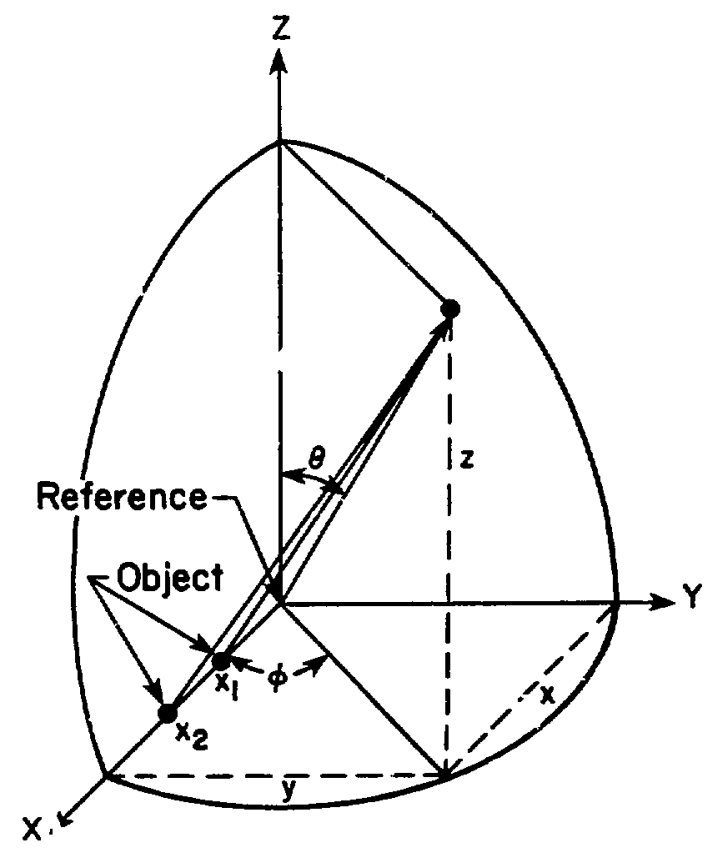

Fig. 5.

Geometry of spherical Fourier transform holography on a sphere of unit radius. 
IV. RECORDING METHODS FOR FRESNEL TRANSFORM HOLOGRAPHY

To obtain indefinitely high resolution, Fresnel transform holography requires a grainless recording medium. No recording medium is truly grainless, it is at least grainy on the atomic scale. However, there are ways of obtaining resolution far better than that available from the finest-grain film. One is to convert the $x$-ray image into an electron image by means of a photo-emitting cathode, and then enlarge the image with an electron microscope. This has the additional advantage that the electron microscope could be time-gated, thereby extracting information only during the part of the laser pulse when the best hologram was being generated. The other approach is to use a high-resolution recording medium. This could be done using a photoresist material, many of which have been developed for high-resolution lithography. 32

\section{A. Piintoresist Recording}

An example of such a material is polymethyl methacrylate (PMMA). Photons striking this plastic break polymer chains leaving the material locally less resisiant to etching agents. Feder et al. ${ }^{33}$ have demonstrated that resolution of $100 \AA$ can be obtained with PMMA and resolutions of $50 \AA$ have been rumored. We àre most interested in wavelengths in the 30-50 $\AA$ range. This is optimum for the use of a photoresist such as PMMA. At higher energies the resolution degrades because of the increasing range of secondary electrons, and at lower energies the resolution degrades because of diffraction effects. ${ }^{3}$ Holograms registered on a photoresist can be read out using a transmission electron microscope and the image can be reconstructed either by optical laser illumination of the electron micrograph or computer analysis. ${ }^{2}$ Specially prepared polymer photoresists can be used effectively for recording exposures down to $10^{-2} \mathrm{~J} \cdot \mathrm{cm}^{-2}$, and perhaps below at some sacrifice of quantum efficiency. ${ }^{32}$

Recording surfaces using a thin layer of silver halide, similar to Daguerreotype plates, ${ }^{34}$ have demonstrated sensitivities to $x$-ray exposure better than $10^{-2} \mathrm{~J} \cdot \mathrm{cm}^{-2}$. Edge widths observed with a scanning electron microscope are resolved to better than $5000 \AA$, and the technique may ultimately become competitive with photoresists. 


\section{B. Electron Microscope Recording}

Direct recording with an electron microscope imposes a trade-off between quantum efficiency and resolution. The modulation transfer function degrades with increase in acceptance angle and energy dispersion of the photoelectrons. ${ }^{35}$ The spherical aberration of an electron microscope increases as the cube of its acceptance angle. ${ }^{36}$ While the quantum efficiency decreases as the square of the acceptance angle. Similarly, the chromatic aberration increases as the product of acceptance angle and energy dispersion. ${ }^{36}$ We might reduce the chromatic aberration by using electronic gating to limit the energy dispersion, but the problem of spherical aberration is not easily circumvented.

It is possible to build lensless electron microscopes. One such concoptual device using a Fresnel transform setup is shown in Fig. 6. The photoemitter consists of a spherical shell, radius $r_{1}$, and the recording surface consists of a spherical shell at a much larger radius $r_{2}$. The magnification should be given by the ratio of $r_{2} / r_{1}$. An accelerating high voltage is applied to the inner shell while the outer shell is grounded. The electrons are accelerated nearly radially and blurring is owing exclusively to initial tangential velocities. However, if the energy corresponding to the initial tangential velocity is $E_{0}$ and the potential on the inner sphere is $V_{0}$ then the dispersion in angle [see Appendix C] is given by

$$
\Delta \theta \cong 2\left(\frac{r_{2}-r_{1}}{r_{2}}\right) \sqrt{\frac{E_{0}}{v_{0}}}
$$

This approximation is good when $\mathrm{E}_{0}<\mathrm{V}_{0} \times 10^{-3}$. The detailed expression is very complicated.

Examining this expression we immediately notice that the case of least blurring occurs as $r_{1}$ approaches $r_{2}$. This is the case of least magnification, i.e., parallel planes. However, in accelerating the electrons we have also decreased the divergence angle by a factor of $\sqrt{E_{0}} / V_{0}$. So we might instead consider using parallel plates to accelerate the electrons into the optics of a conventional electron microscope, and gain resolution by decreasing the acceptance angle. 


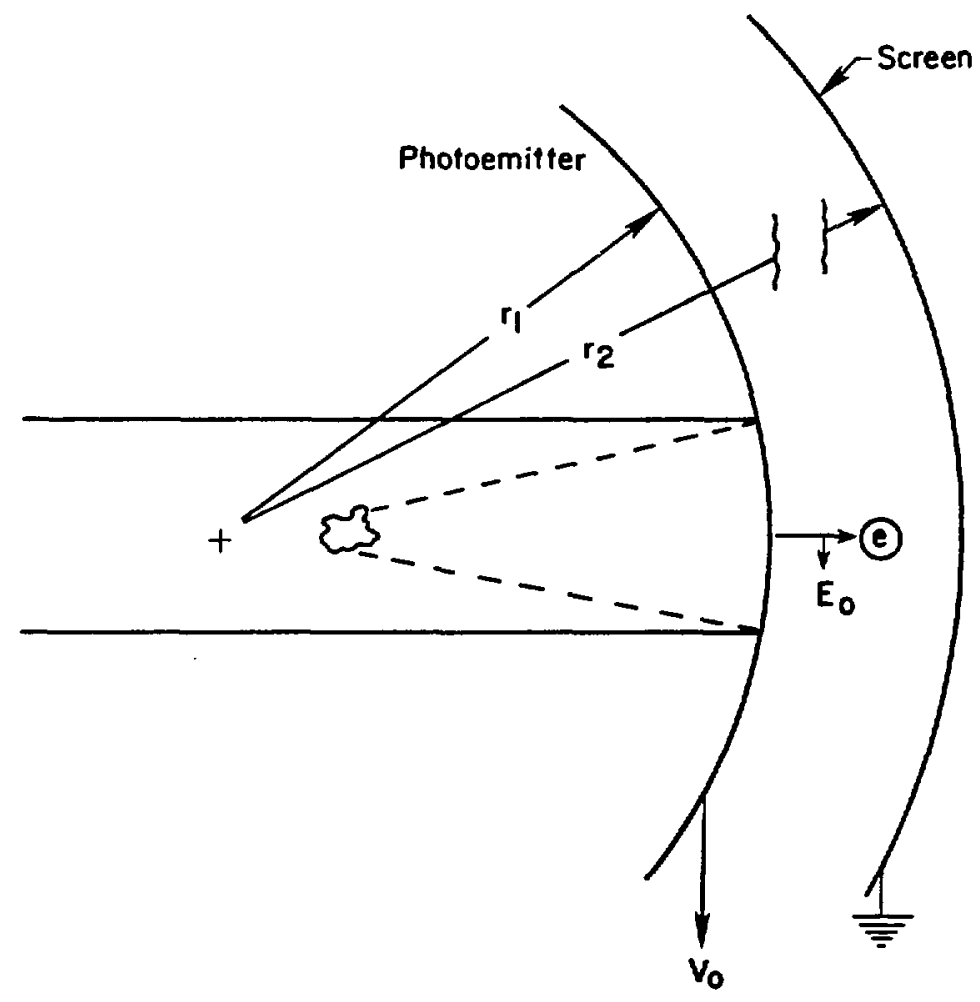

Fig. 6.

Conceptual lensless electron microscope with Fraunhofer holograph.

However, it is difficult to conceive of any practical conisuration that would result in an acceptable blurring. For example, if we want the blurring, $r_{2} \Delta \theta$, to be only $100 \AA$, and we have a distance between plates, $r_{2}-r_{1}$, of only $10 \mu \mathrm{m}$, and a tangential energy of only 1 volt, then we need 4000000 volts between the plates. This is clearly impractical.

It may be possible to use computer image-enhancement techniques to deblur the image, because we have good theoretical knowledge of the source function. This possibility is still under investigation.

C. Shadow Foils

A potential problem with Fraunnofer holography is low contrast ratio. This is particularly true if the specimen is not a very efficient coherent scatterer. 
The problem can be circumvented by inounting the specimen over a hole in an absorbing foil, which will attenuate the reference beam. The uniformity of the "shadow foil" must be such that spatial and temporal coherence of the reference beam are not drastically altered.

\section{REALIZATION OF FOURIER TRANSFORM HOLOGRAPHY AT X-RAY WAVELENGTHS}

\section{A. Planar Fourier Transform}

The trouble with lensless Fourier holography is that (in the figurative sense) it is not lensless. Figure 7 shows a typical setup for planar Fourier holography. In order to obtain the spherical reference wave we must have a lens that focusses to a pinhole in the shadow plane. In the $x$-ray regime we do not use a lens, but rather use a Fresnel zone plate. It is easy to prove [see Appendix B] that with this type of experimental configuration, the hologram resolution is limited to the finest spacing on the Fresnel zone plate. This derives from the fact that the finest spacing on the zone plate determines the maximum angle of the spherical segment generated. The finest zone plate spacing allowed by current technology is about $800 \AA .37$ This means that using this technique we could obtain resolution no better than $800 \AA$.

\section{B. Spherical Fourier Transform}

An alternative could be to use a coherent scattering reflector to generate the reference waves. ${ }^{38}$ Figure 8 shows such a reflector, in this case a paraboloid that would generate spherical reference waves, enclosed in a spherical shell recording surface. For best contrast ratio, the paraboloid would have to be approximately the same size as the object (for example, $100 \mu \mathrm{m}$ for a $100 \mu \mathrm{m}$ biological specimen). The reference scatterer need not be a paraboloid. In principle, the hologram could be unfolded for any convex reference scatterer as long as we knew its shape and dimensions to within a fraction of a wavelength. 14 Presently, spherical microballoons can be made $100 \mu \mathrm{m}$ in diameter with a surface smoothness better than $100 \AA$, and these balloons can be measured to within $50 \AA .^{39}$ So using a reflector that would work in the 30-50 $\AA$ region is just on the edge of technological feasibility. 


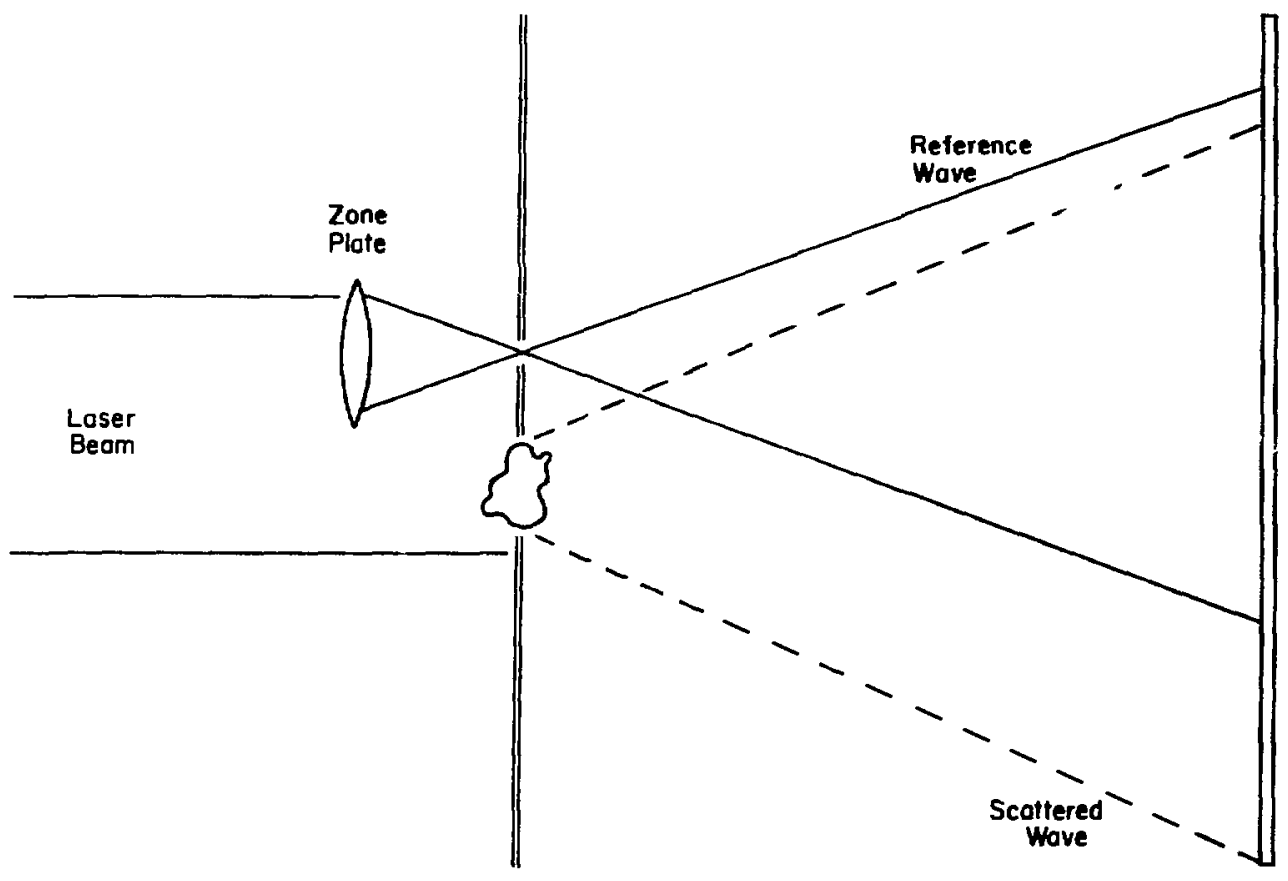

Fig. 7.

Typical setup for planar Fourier transform holography.

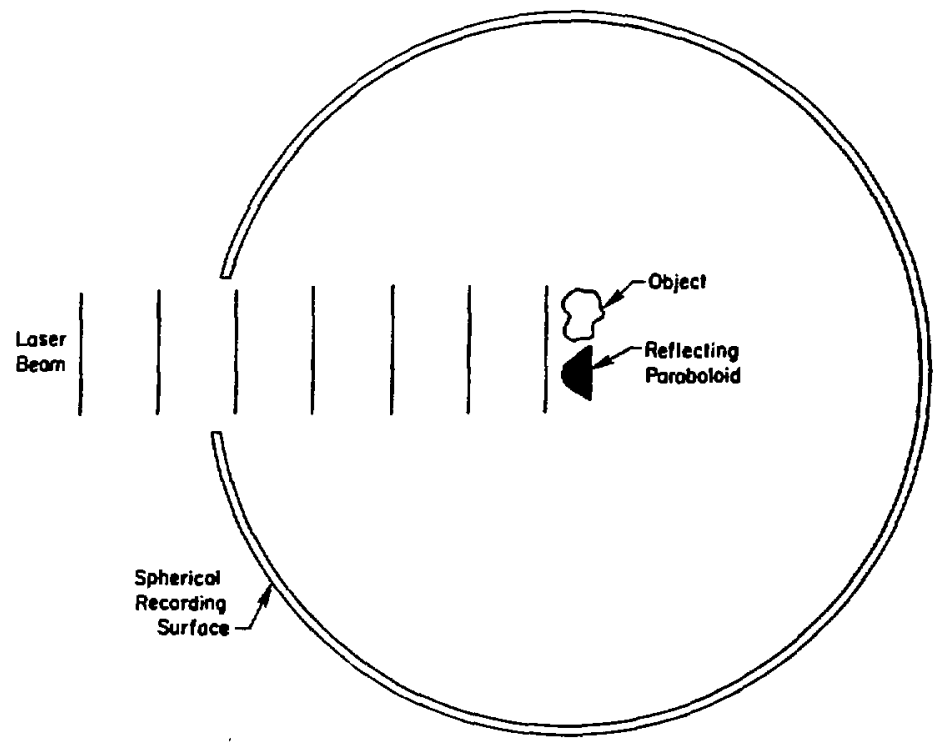

Fig. 8.

Spherical Fourier transform holography with a parabolic reflector. 


\section{Recording Media for Fourier Transform}

In Fourier transform holography, the fringe spacing can be made arbitrarily large by adjusting the distance between the reference source and the recording surface. The linear resolution of the recording surface required for the reconstructable registration of the hologram varies directly as this distance, while the required sensitivity of the recording surface varies inversely as the square of this distance. The figure of merit of a recording medium used for Fourier transform holography is therefore inversely proportional to the product of its sensitivity $\left(\mathrm{J} \cdot \mathrm{cm}^{-2}\right)$ and the square of its grain size $\left(\mathrm{cm}^{2}\right)$.

It is not clear that film is superior to photoresist for this application. For example, the highest resolving-power $x$-ray films have a grain size of about $5 \mathrm{\mu m}$. One such film is Kodak PAR 2490, which gives an optical density $\left(\log _{10}\right.$ [incident light/transmitted light]) of $\frac{1}{2}$ for an exposure of 0.176 photons $\cdot \mu^{-2}$ at a quantum energy of $400 \mathrm{eV} .{ }^{40}$ This corresponds to a figure of merit of $\sim 10^{14} \mathrm{~J}^{-1}$. Because the sensitivity of photoresists is measured in a different way, it is difficult to make a direct comparison with film. Normally, a photoresist sensitivity is given by the fraction of original material that remains in unexposed areas after the exposed areas have been completely etched away. ${ }^{31}$ This is the most reasonable measure for lithographic purposes. To compare with film, the logically compelling measure is the optical density of the photoresist in a transmission electron microscope. No such measurements have been published, but a reasonable estimate is $10^{-2} \mathrm{~J} \cdot \mathrm{cm}^{-2}$ for sensitivity with a linear resolution of $100 \AA$, which gives a figure of merit of $\sim 10^{14} \mathrm{~J}^{-1}$. In other words, film and photoresist are roughly equal as recording media for Fourier transform holography at the most interesting wavelengths for biological application--certainly neither is better by an order of magnitude. This is consistent with a long held notion in the photographic sciences that the average size of latentimage speck required for developability, and the average number of absorbed quanta required to form a developable speck, are independent of grain size. ${ }^{41}$

An alternative is to use an $x$-ray photocathode and microchanneltron for recording a Fourier transform hologram. The best photocathode at $400 \mathrm{eV}$ is CsI, which has a quantum yield near unity. ${ }^{42}$ Because the secondary electron energy distribution of $\mathrm{CsI}$ is very sharp, 42 the recording system could be gated on the time scale of picoseconds--a feature which has important implications for defeating hydrodynamic blurring if the length of the laser pulse cannot be well controlled. Microchannelplates are available with linear resolution of about 
$50 \mu \mathrm{m}$. They have a a gain of about $10^{4}$, so essentially every photoelectron is recorded. 43 Unfortunately they saturate easily and usually have a dynamic range of $\sim 40$. Furthermore, they are generally available only in fairly small sizes $(\sim 0.4 \mathrm{~cm})$, and larger plates are difficult to manufacture reliably.

In figure of merit, however, the photocathode-microchanneltron is far superior to buth film and photoresist. From the estimates above, the figure of merit is $\sim 10^{18} \mathrm{~J}^{-1}$, about four orders of magnitude better than film or photoresist. This technology should therefore be considered carefully in conjunction with the Fourier transform technique.

\section{COMPARISON OF HOLOGRAPHIC TECHNIQUES}

Table III compares the holographic techniques I have discussed in the previous sections.

TABLE III

COMPARISON OF HOLOGRAPHIC TECHNIQUES

\begin{tabular}{|c|c|c|c|}
\hline $\begin{array}{l}\text { Type } \\
\text { Generic } \\
\end{array}$ & \multicolumn{2}{|c|}{ Fresnel Transform } & Fourier Transform \\
\hline Specific & On-Axis & Off-Axis & Spherical \\
\hline Associated Nane & Gabor & Leith-Upatnieks & Skrinsky \\
\hline Figures & $1 \mathrm{a}, 2$ & $2 b, 3,15$ & $1 c, 4,7$ \\
\hline Gecmetry & \multirow{2}{*}{\multicolumn{2}{|c|}{ Plane }} & \multirow[b]{2}{*}{ Spherical } \\
\hline $\begin{array}{l}\text { Reference } \\
\text { Wavefront }\end{array}$ & & & \\
\hline $\begin{array}{l}\text { Reference } \\
\text { Surface }\end{array}$ & \multicolumn{2}{|r|}{ Plane } & Spierical \\
\hline $\begin{array}{l}\text { Reference } \\
\text { Source }\end{array}$ & Distant, In-Line & Distant, off-axis & $\begin{array}{l}\text { Point or Equivalent (Zone Plate or } \\
\text { Parabolic Reflector) }\end{array}$ \\
\hline Advantage & Simplicity & $\begin{array}{l}\text { Separatiun of } \\
\text { Real and Virtual } \\
\text { Images }\end{array}$ & $\begin{array}{l}\text { Less Sensitivity to Grain Size } \\
\qquad \begin{array}{|l}\text { (1) } 4 \pi \text { Recording } \\
\text { (2) Less Sensitive } \\
\text { to Recording } \\
\text { Surface Rough- } \\
\text { ness }\end{array}\end{array}$ \\
\hline Difficulties & \multicolumn{2}{|c|}{ Resolution Limited by Grain Size } & \begin{tabular}{l|l} 
Survivable High & Well Characterized \\
Precision Zone & Reflector \\
Plate &
\end{tabular} \\
\hline Registration & $\begin{array}{l}\text { Temporal Coherenc } \\
\text { Requirements }\end{array}$ & $\begin{array}{l}\text { Mirror } \\
\text { Requirements }\end{array}$ & Tradeoff between sensitivity and $\Delta^{2}$ \\
\hline Reconstruction & \multicolumn{2}{|c|}{$\begin{array}{c}\text { Obscuration by Virtual leage Unless } \\
b \lambda \gg d^{2} \mid \theta_{r}>\sin ^{-1}(\lambda / \delta)\end{array}$} & Dynamic Reference Wavefront Distortion \\
\hline References & $10 ; 11$ & 31 & $14,15,16$ \\
\hline
\end{tabular}


VII. HYDRODYNAMIC BLURRING MECHANISMS

The differences in average absorption and scattering cross sections of various regions within a cell make interpretable holography possible. However, because of the heating that attends absorption, these very inhomogeneities within the specimen also give rise to hydrodynamic disturbances that result in blurring of the hologram. For example, if we are trying to record the density of nitrogen, and selecting a wavelength that will give preferential absorption to nitrogen atoms or nitrogen bonds within the biological structure, then there will be a tendency for regions containing a high concentration of nitrogen to expand more rapidly than those regions containing lower concentrations. Protein contains an order of magnitude more nitrogen than other cellular constituents. Under laser irradiation we would expect these concentrations of protein to explode, driving shockwaves into the surrounding medium. Protein molecules within the lipid bilayers of membranes would explode rupturing the membranes and displacing the cytoplasm at their borders. In small prokaryotic cells, protein concentrations on the ribosomes would suffer similar explosion.

The exact way in which the explosion of absorbing material will affect the blurring of the hologram is dependent on the details of the structure being examined and the relative concentrations of absorbing material. In Appendix D we derive an analytic treatment for the early-time explosion and the late-time expansion of the concentrations. These have been compared to numerical hydrodynamic calculations using Lagrangian techniques. ${ }^{44}$ While the analytic solutions provide some feel for the sensitivity to adjustable variables in a broad range of problems (which can be used to optimize the resolution of the hologran) a true examination of any specific problem requires a case-by-case numerical calculation of a mock-up of the configuration to be holographed.

\section{A. Explosions in a Transparent Medium}

Under the assumption that the laser beam energy is deposited only in a sphere of initial radius $R_{0}$ (which $I$ call a protsin globule for convenience), and that the only way energy is transferred from the protein globule to the surrounding material (which I call cytoplasm for convenience) is by mechanical work, we find from Appendix D the following description of the protein radius as a function of time. 
Early Time

$$
R_{p}=R_{0}+\left[f(\gamma) K \Sigma_{a b s} I\right]^{1 / 2} t^{3 / 2}
$$

where $f(\gamma)=\frac{2}{3} \frac{(\gamma-1)}{(\gamma+1)^{2}}$.

Late Time

$$
R_{p}=\left[g(\gamma) K \Sigma_{a b s} I\right]^{1 / 5}\left(R_{0} t\right)^{3 / 5}
$$

where $g(\gamma)=\frac{25}{4} \frac{(\gamma+1)\left(\gamma^{2}-1\right)}{(8 \gamma-1)}\left[\frac{2}{\gamma+1}\right]^{5 / 3}$.

The transition from Eq. (4) to Eq. (5) occurs at $R_{p} \cong 7 / 4 R_{0}$. The displacement of the radius does not depend on the initial radius in $\mathrm{Eq}$. (4), suggesting that the measure of linear blurring is independent of the size of the globule, as long as it is not large compared to the globule.

The fact that the inhomogeneities in the material that is absorbing laser energy may not be spherical in shape does not affect the utility of Eqs. (4) and (5), for displacement in Eq. (4) is independent of initial radius, and by the time Eq. (5) comes into play, the expansion of nonspherical shapes into a homogeneous medium will become roughly spherical. Furthermore, protein molecules in the membranes of cells and mitochondria appear to be nearly spherical when observed by state-of-the-art electron microscopy.

\section{B. The Effect of Electron Transport}

Appendix $F$ discussed various mechanisms by which a laser heated region can transfer energy to the surrounding medium other than hydrodynamically. The fact that other mechanisms exist make the results given in Eqs. (4) and (5) an overestimate of the actual blurring. Of the mechanisms discussed in Appendix $G$, the most serious is electron transport. The reason is that if in general we tune the laser to single out an individual atomic species, it is most likely that we will use a $\mathrm{K}$-edge or a resonance in the vicinity of the K-edge. In our energy 
range, the most likely mode of de-excitation of an absorbing atom is by Auger transitions releasing energetic electrons. Appendix G derives formulas parallel to Eqs. (4) and (5) for the expansion of a protein globule whose atoms de-excite by emitting fast electrons that may penetrate into the surrounding cytoplasm. In terms of the range of the fast electrons $\eta$ (assumed the same in protein and cytoplasm) they are the following.

Early Time

$$
R_{p}=R_{0}+\left[F(\gamma, \eta) K \Sigma_{a b s} I\right]^{1 / 2} t^{3 / 2}
$$

where $F(\gamma, \eta)=\frac{2(\gamma-1)}{3(\gamma+1)^{2}(1+\zeta)^{5}}$ and $\xi=\frac{\eta}{\rho_{0} R_{0}}$.

Late Time

$$
R_{p}=\left[G(\gamma, \eta) K \Sigma_{a b s} I\right]^{1 / 5}\left(R_{0} t\right)^{3 / 5}
$$

where $G(\gamma, \eta)=\frac{250}{81} \frac{\left(\gamma^{2}-1\right)}{(1+\zeta)^{6}} \zeta q^{-10 / 3}+\frac{25}{4} \frac{(\gamma+1)\left(\gamma^{2}-1\right)}{(1+\zeta)(8 \gamma-1)}\left[\frac{2}{\gamma+1}\right]^{5 / 3}$.

The constant $q$ has a value that depends on the radius at which the outgoing shockwave makes a transition to a detonation: $q \cong 1.6180$ if the transition radius is taken to be when the stopping power of the snowplow region (the higher density shell generated by shock compression) is equal to the stopping power of the lower density shocked material in the sphere within the snowplow region. The value of $q$ should actually be determined from comparison with numerical calculations.

\section{INTENSITY REQUIREMENTS}

In the previous section we have discussed the hydrodynamic motion of components of the specimen that will give rise to blurring. In general, we must 
have a high enough intensity laser and a short enough pulse length (or fast enough shutter speed at the recording surface) to keep this blurring effect within acceptable bounds.

Let us assume we are trying to make a hologram of a relatively transparent medium in which we have imbedded local concentrations of a material that has a large total cross section for the laser frequency we are using. For the sake of having a concrete reference point, we will continue with the problem of main inturest, imaging the density of protein. In a typical eukaryotic cell, e.g., rat liver, we would expect most of the protein to appear in the form of nearly spherical shaped molecules in lipid bilayers in the cell membrane and in the membranes of mitochondria. These molecules have a typical molecular weight of $3-$ to- $4 \times 10^{4}$ and would be about $25 \AA$ in radius. Protein may also concentrate in the cytoskeleton and structural elements of the nucleus, and may be found in concentrations of considerably larger dimension in fibrous form. For example, the fibrous form collagen is the main constituent of skin, tendons, and connective tissue.

\section{A. Optimum Exposure Time}

If we are trying to make a hologram wi h a linear resolution of $\delta(\mathrm{cm})$, it is necessary that no part of the specimen move more than $\delta$ during the exposure time, $\Delta t$. If $\delta$ is smaller than the radius of a typical protein globule, then the early-time formula [Eq. (6)] should be used. Taking the radial displacement as $\delta$ we can rewrite $\mathrm{Eq}$. (6) to give the optimum exposure time as

$$
\Delta t=\frac{\delta^{2 / 3}}{\left[F(\gamma, \eta) K \Sigma_{a b s} I\right]^{1 / 3}}
$$

Equation (8) is the optimum exposure time in the sense that it is the maximum length of exposure that will insure a hydrodynamic blurring less than $\delta$. If the linear resolution is to be much larger than the physical dimension of the protein globule, we must use Eq. (7) to obtain the optimum exposure time, which when appropriately rearranged becomes 


$$
\Delta t=\frac{\delta^{5 / 3}}{R_{o}\left[G(\gamma, \eta) K \Sigma_{a b s} I\right]^{1 / 3}} .
$$

The optimum exposure times given by Eqs. (8) and (9) could be achieved by designing a laser with a natural short pulse or by using any of a variety of mechanisms for chopping the pulse into smaller pieces. A gated recorder such as any of the electron photoemissive schemes, could also provide a shutter and allow the laser pulse to exceed the optimum exposure time without blurring the hologram. With a reflective-reference scheme such as that shown in Fig. 8 we could select a coherent scattering resonance and laser intensity such that the resonance bleached in a time close to the optimum exposure time. The same resonance would be used in both the reference reflector and the object, and would provide a natural shutter. When the resonance bleached, both the object and reference scatterer would become transparent to the laser beam, and generation of the hologram would cease. Unfortunately, this shutter scheme cannot be used effectively with Fresnel transform holography and a photoresist recording surface, for after the resonance bleaches, the laser will continue to expose (darken) the photoresist. It would be permissible to let the laser pulse length exceed the saturation time by a factor of 2 or 3 , but any further exposure would unacceptably degrade the contrast ratio.

Keeping in mind a model for the specimen, which resembles an array of protein globules imbedded in a relative transparent medium, the number of photons recorded per resolution element is given by

$$
\mathrm{N}=\left\{\int \mathrm{dV} \exp \left(-\mathrm{K} \Sigma_{\text {tot }} \rho \Delta \mathrm{x}\right) \exp \left(-\mathrm{K} \Sigma_{\text {tot }} \rho \Delta \mathrm{x}^{\prime}\right) \mathrm{K} \Sigma_{\text {elas }} \rho\right\} \frac{\varepsilon \mathrm{I} A \Delta \mathrm{t}}{\mathrm{hv}}
$$

where $\Delta \mathrm{x}$ and $\Delta \mathrm{x}^{\prime}$ are the distances from the surface of the cell to the volume element $d V$ along the incident and scattered paths, $h v$ is the photon quantun energy (erg), $\varepsilon$ is the detector quantum efficiency, i.e., the fraction of photons recorded, $\Sigma$ elas is the elastic cross section of a protein globule $\left(\mathrm{cm}^{2}\right.$. globule $\left.{ }^{\top}\right), \Sigma_{\text {tot }}$ is the total cross section of a protein globule $\left(\Sigma_{\text {tot }} \cong \Sigma_{\text {elas }}\right.$ $\left.+\Sigma_{a b s}\right)$, and $V$ is the volume of a resolution element. If we assume that the 
$x$-ray thickness of the specimen is not so great as to prevent many of the photons from reaching the resolution elements or to prevent scattered photons from escaping the specimen, this equation can be simplified to:

$$
\mathrm{N} \cong \frac{\varepsilon}{\mathrm{h} v} \mathrm{~K} \Sigma_{\text {elas }} \rho \mathrm{I} \delta^{3} \Delta \mathrm{t}
$$

Since the density times the volume of any volume element is just the mass and remains fixed as the volume expands, we can replace $\rho$ with $\rho_{o}$ for scattering purposes.

\section{B. Intensity}

If the $x$-ray laser is the only heat source causing hydrodynamic blurring, we may use the optimum exposure times given by Eqs. (8) and (9) for the pulse length in $\mathrm{Eq}$. (12). Equation (12) may then be easily solved for the intensity requirements. The intensities are:

$$
I \cong \frac{1}{\mathrm{~K}} \sqrt{\left.\frac{\mathrm{N} \mathrm{hv}}{\varepsilon \Sigma_{\text {elas }} \rho_{\mathrm{o}}}\right)^{3} \frac{\mathrm{F}(\gamma, \eta) \Sigma_{\mathrm{abs}}}{\delta^{11}}}
$$

if $\delta<\frac{3}{4} R_{0}$

or

$$
I \cong \frac{1}{\bar{K}} \sqrt{\left(\frac{N \text { hv } R_{o}}{\varepsilon \Sigma_{\text {elas }} \rho_{o}}\right)^{3} \frac{G(\gamma, \eta) \Sigma a b s}{\delta^{14}}}
$$

if $\delta \geq \frac{3}{4} R_{0}$.

These are very powerful and versatile formulae, not so much because they give the laser intensity required to obtain any desired resolution as a function of measurable parameters, but because they can be very helpful in aiding intuition. The decision to use Eq. (13) or Eq. (14) depends on the resolution sought 
compared to the size of features that explode under laser heating. Although the formulae were derived assuming spheres of radius $R_{0}$, they are good approximations for features of all shapes. Part of the reason for their versatility is that a small feature becomes spherical in shape after expanding a few times its original dimension regariless of its original shape. Some attention must be paid to geometry if there are clusters of exploding globules. For example, protein molecules in the convolutions of mitochondria membranes may expand far enough to overlap and coalesce into a single expanding sphere. This will change the value of $R_{0}$ to be used in Eq. (14). Equation (13) is pretty much independent of shape of the features that explode as long as the features are large compared to the linear resolution.

The required intensity goes as $\delta^{11 / 2}$ in $\mathrm{Eq}$. (13) and $\delta^{-7}$ in $\mathrm{Eq}$. (14). This very strong dependence on $\delta$ gives a feel for the applicability of $\tilde{F}_{:} \mathbf{s}$. (13) and (14). Even though other parameters may be measured poorly, if, ae equations predict a certain resolution at a given intensity, it is unlikely that the resolution will be off by a factor of 2 . Similarly if we observe a certain resolution in an experiment, we will need about 100 times the laser intensity to double that resolution in the next experiment. The strong inverse dependence on $\delta$ derives from the increase in intensity required to get the same number of photons scattered off a smaller resolution element in a shorter time. Equations (13) aud (14) are applicable only in the region where the specimen is behaving like a fluid and there is local thermodynamic equilibrium.

\section{Meaning of the Cross Sections}

We have taken care to specify $\Sigma_{\text {abs }}$ and $\Sigma_{\text {elas }}$ as the total absorption and elastic cross sections for the entire exploding feature. If we considered a feature consisting of a single atom, we would use the microscopic cross sections for that atom, such as are shown in Fig. 9. Almost everywhere in Fig. 8 the coherent cross section is about three orders of magnitude less than the absorption cross section. This will give discouraging results for the required intensity as specified by Eqs. (13) and (14). Suppose, for example, we were trying to make a hologram to a linear resolution of $100 \AA$ of a microscopic specimen containing of our standard protein. Further, suppose that we wanted to scatter coherently $10^{3}$ photon. from each resolution element. Using nonresonant coherent scattering, the elastic cross section is about $4 \times 10^{-22} \mathrm{~cm}^{2}$. The absorption cross section is about $4 \times 10^{-20} \mathrm{~cm}^{2}$. The required intensity is 

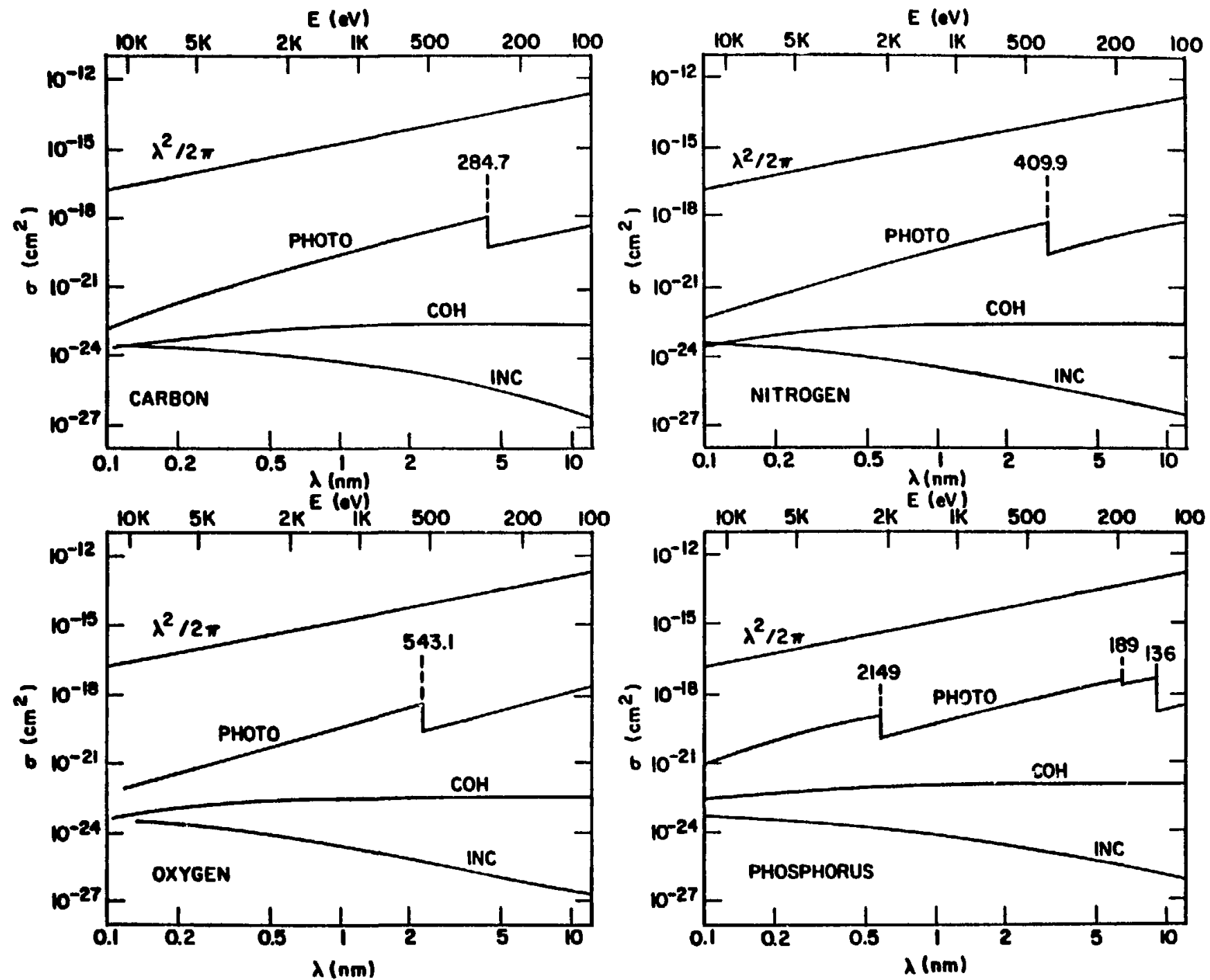

Fig. 9.

Cross sections for interaction of photons with atoms of four biologically important elements, in the 0.1-10 nm region of wavelength. Legend: "PHOTO", photoelectric absorption; "COH", coherent or elastic scattering; "INC", incoherent or inelastic scattering; " $\lambda^{2} / 2 \pi^{1}$, the maximum possible cross section for a resonant interaction. 
about $4.4 \times 10^{16}$ watts $\cdot \mathrm{cm}^{-2}$ and the exposure time is about $5 \times 10^{-13} \mathrm{~s}$. This is an uncomfortably high intensity and an exceedingly short pulse length.

The actual situation is not as difficult as this suggests. We are not trying to look at individual atoms, but globules of protein. When a large number of atoms are gathered trgether in a package thick enough to substantially attenwate the $x$-ray beam traversing it, the elastic scattering cross section of the whole package may become much larger than the sum of the elastic cross sections of the individual atoms. When the object becomes thick enough to absorb all of the incident radiation, both its elastic and absorption cross section converge to the geometric cross section as long as the wavelength is small compared to the linear dimensions. This limiting case can be solved classically, 45 for example, for a perfectly absorbing sphere,

$$
\Sigma_{\text {abs }}=\Sigma_{\text {elas }}=\Sigma_{\text {geometric }}=\pi a^{2}
$$

where a is the radius of the sphere. The limiting cases for Eqs. (13) and (14) are

$$
I \cong \frac{1}{\pi R_{0}^{2} K} \sqrt{\left(\frac{N h v}{\varepsilon \rho_{0}}\right)^{3} \frac{F(\gamma, \eta)}{\delta^{1 I}}}
$$

if $\delta<\frac{3}{4} R_{0}$, and

$$
I \cong \frac{1}{\pi K} \sqrt{\left(\frac{N h v}{\varepsilon \rho_{o}}\right)^{3} \frac{G(\gamma, \eta)}{R_{o} \delta^{14}}}
$$

if $\delta \geq \frac{3}{4} R_{c}$; and the corresponding optimum exposure times are

$$
\Delta t=\frac{\gamma^{5 / 3}}{\left[F(\gamma, \eta) K \pi R_{0}^{2} I\right]^{1 / 3}}
$$


and

$$
\Delta t=\frac{\gamma^{5 / 3}}{\left[G(\gamma, \eta) K \pi R_{0}^{5} I\right]^{1 / 3}} .
$$

These formulae represent limiting values, and there is a slight logical contradiction in using them. Equations (6) and (7) assume a uniform heating of the globule of radius $R_{0}$, which is not possible if the globule approaches a perfect absorber.

D. The Intermediate Case

We are most interested in the case where our features are not completely opaque. If we tune the laser to a nitrogen resonance in protein analogous to the IS $\rightarrow \pi 2 \mathrm{P}$ transition in $\mathrm{N}_{2}$ (see Appendix $\mathrm{A}$ ), we could expect an atomic absorption cross section of $\sigma_{\text {abs }}=1.7 \times 10^{-17} \mathrm{~cm}^{2}$ for each nitrogen atom. If we take $\rho_{0}=1 \mathrm{gm} \cdot \mathrm{cm}^{-3}$, then the e-folding length for laser intensity is about $693 \AA$. In other words, structures of protein about $700 \AA$ thick will attenuate the beam a. factor of 3 .

The theory of scattering for the intermediate case has been worked out by Glauber for the elastic cross sections of heavy nuclei. 46 The many atoms in a protein globule are analagous to the many nucleons in a heavy nucleus. Glauber's theory is complex, but the principal feature can be stated rather simply: the elastic cross section is approximately equal to the geometric cross section times the square of the extinction. By the extinction we mean the fraction of the beam removed by all processes. In other words,

$$
\Sigma_{\text {elas }} \cong \operatorname{Min}\left[\rho R_{0}^{2}, \operatorname{Max}\left\{\frac{4}{3} \pi \kappa \rho_{o} R_{o}^{3} \sigma_{e l a s},\left[1-\exp \left\{-\kappa \sigma_{\text {tot }} \rho_{o}\left(\overline{2 R}_{o}\right)\right\}\right]^{2} \pi R_{o}^{2}\right\}\right]
$$

where $\sigma_{\text {elas }}$ and $\sigma_{\text {tot }}$ are the cross sections of the absorbing atoms, $k$ is the number of absorbing atoms per gram, and $\left(\overline{2 R}_{0}\right)$ is the properly averaged secant through the globule. This is a highly simplified version of the actual form 


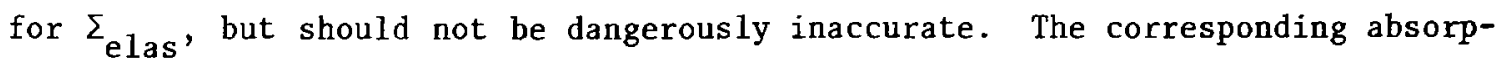
tion cross section is

$$
\Sigma_{\text {abs }} \cong\left\{\pi \mathrm{R}_{\mathrm{o}}^{2}\left[1-\exp \left\{-\kappa \sigma_{\text {tot }} \rho_{0}\left(\overline{2 R}_{\mathrm{o}}\right)\right\}\right], \frac{4}{3} \pi \kappa \rho_{\mathrm{o}} \mathrm{R}_{\mathrm{o}}^{3}\right\}
$$

and the same caveats apply.

IX. INTENSITY AND PULSE IENGTH CALCULATIONS

\section{A. Short Pulse Holography}

We are now prepared to calculate the required intensity and exposure time for some examples.

Problem 非. We are trying to holograph an array of protein structures of typical dimension $1000 \AA$ to a linear resolution of $100 \AA$. The structures are imbedded in a transparent cytoplasm-like medium, and we are using a resonant transition analogous to the one shown in Fig. 2. We have a unit quantum efficiency and signal-to-noise considerations require that we record $10^{3}$ photons from each resolution element. What is the required intensity, exposure time, and exposure of the recording surface?

Solution. $\delta=100 \AA$ and $R_{0} \cong 500 \AA$, so we should use Eq. (8) for the exposure time and $\mathrm{Eq}$. (13) for the intensity. For the $1 \mathrm{~S} \rightarrow \pi 2 \mathrm{P}$ transition, $\sigma_{\text {abs }} \cong \sigma_{\text {tot }} \cong 2.2 \times 10^{-17} \mathrm{~cm}^{2}$ and $\sigma_{\text {elas }} \cong 10^{-20} \mathrm{~cm}^{2}$. We can estimate $\left(\overline{2 R}_{0}\right) \cong$ $\frac{4}{3} R_{o}$, so from Eq. (19)

$$
\Sigma_{\text {elas }} \cong 3.0 \times 10^{-11} \mathrm{~cm}^{2}
$$

and from Eq. (20)

$$
\Sigma_{\mathrm{abs}} \cong 4.9 \times 10^{-11} \mathrm{~cm}^{2}
$$


Now most of the energy from the absorbed radiation will be converted to fast electrons because we are using an autoionizing transition. Say the typical electron energy is $350 \mathrm{eV}$. The range of a $350-\mathrm{eV}$ electron in water is about $3 \times 10^{-7} \mathrm{gm} \cdot \mathrm{cm}^{-2}$, so $\eta \cong 30 \AA$ and $\zeta \cong 0.06$ and we might guess $\gamma \cong 1.4$. From Eq. (6),

$$
F(\gamma, \eta)=0.035
$$

The density of protein is about $1 \mathrm{gm} \cdot \mathrm{cm}^{-3}$, so $\mathrm{K} \cong 1.9 \times 10^{15}$ structures per gram (see Appendix A). Now hv $\cong 6.43 \times 10^{-10} \mathrm{ergs}$ and $\mathrm{N} / \varepsilon=10^{3}$, so from Eq. (13)

$$
\mathrm{I} \cong 2.2 \times 10^{11} \mathrm{~W} \cdot \mathrm{cm}^{-2},
$$

a rather modest intensity, and from Eq. (8)

$$
\Delta t \cong 5.2 \times 10^{-12} \mathrm{~s}
$$

If the scattering efficiency (ratio of the intensity impinging the specimen to the scattered intensity received at the recording surface) is $10^{-2}$, and an appropriate shadow foil is used to attenuate the reference beam, then the exposure of the recording surface is about $10^{-2} \mathrm{~J} \cdot \mathrm{cm}^{-2}$, near the minimum range for photoresists.

Problem \#2. We want to holograph a cell membrane by resonating with the nitrogen in globular protein molecules $\left(A \cong 4 \times 10^{5}\right.$ ) imbedded in the lipid bilayer. We want a resolution of $100 \AA$, and estimate that the molecules will not overlap owing to their expansion during the exposure time. Again, signal-tonoise considerations suggest that we need $N / \varepsilon=10^{3}$. What is the required intensity, exposure time, and exposure of the recording surface? 
Solution. Because the radius of such molecules is $R_{0} \cong 25 \AA$ and we want $\delta=100 \AA$, we must use Eq. (14) for the intensity and Eq. (9) for the pulse length. From Eq. (19),

$$
\Sigma_{\text {elas }} \cong 4.1 \times 10^{-16} \mathrm{~cm}^{2}
$$

and from Eq. (20)

$$
\Sigma_{\mathrm{abs}} \cong 9.0 \times 10^{-15} \mathrm{~cm}^{2}
$$

The same sort of fast electrons will occur from the absorption, so $\eta \cong 30 \AA$, but $\zeta \cong 1.20$. Since there is considerable heating and expansion of the protein molecules, we will guess $y=5 / 3$. From Eq. (7),

$$
G(\gamma, \eta) \cong 0.69
$$

Now $K \cong 1.53 \times 10^{19}$ protein molecules per gram, so from Eq. (14),

$$
\mathrm{I} \cong 4.0 \times 10^{12} \mathrm{~W} \cdot \mathrm{cm}^{-2}
$$

and from Eq. (9)

$$
\Delta t \cong 2.6 \times 10^{-12} \mathrm{~s}
$$

and if the scattering efficiency is again $10^{-2}$, then the recording surface exposure is about $10^{-1} \mathrm{~J} \cdot \mathrm{cm}^{-2}$. 
B. Long Pulse Holography

Although the intensity requirement for the two problems worked in the previous section are quite modest, and the recording surface exposure is near optimum, the exposure times are quite short. This is owing to the fact that the optimum exposure time goes as the inverse 1/3 power of the laser intensity. If the $x$-ray laser pulse length is considerably longer than the optimum exposure time, two approaches can be taken: (1) develop a shutter mechanism that will produce the effect of a shorter pulse with proportionally shorter rise time and fall time; or (2) use a low-intensity iong pulse arranged so that the expansion of inhomogeneities given at late time by Eq. (7) will produce a tolerable resolution hologram.

One can envision several types of shutter schemes with varying degrees of trade-off between effectiveness and complexity. The three most frequently mentioned are: (1) a pinhole that will close off owing to expanding blow-off material; (2) a synthetic Bragg crystal, ${ }^{47}$ perhaps prepared by alternate layers of soap film and high-Z material, 48 that cease to diffract the $x$-ray beam as heating causes the alternating layers to expand; and (3) a saturable absorber such as the resonance discussed in Appendix A that would bleach out after a certain number of bonds were broken. In the first of these schemes, the intensity at the pinhole must be much larger than the intensity at the specimen in order for hydrodynamic motion at the pinhole to close off the pinhole before hydrodynamic motion of inhomogeneities within the specimen blur the hologram. The second scheme is less critical because thermal expansion of the diffracting crystal need only be very slight. The third scheme relies on our being able to calculate the probability of breaking a nitrogen bond per incident photon. While the bleaching effect should be easily calculable, it is very sensitive to intensity. In the long term it may be that the most feasible shutter will combine these techniques or use one that has not been mentioned here.

If a reliable shutter mechanism cannot be developed, and a gateable recording surface such as the electronic schemes discussed in Sec. V are impractical, it may also be possible to make holograms with a long laser pulse. Here is an example.

Problem 非. As in problem 非, we are trying to holograph an array of protein structures of typical dimension $1000 \AA$ imbedded in a transparent cytoplasmlike medium. The only laser available has a pulse length of 100 picoseconds and 
we can shutter neither the laser nor the recording surface. Signal-to-noise considerations will allow $N / \varepsilon=10^{2}$. What resolution can be obtain and what intensity must we use?

Solution. We will assume that we still have $\delta<\frac{3}{4} R_{0}$ so Eq. (6) still describes the hydrodynamic blurring. Rearranging Eq. (8) we have

$$
\delta \cong\left[F(\gamma, \eta) K \Sigma_{a b s} I\right]^{1 / 2}(\Delta t)^{3 / 2}
$$

and substituting into Eq. (12) we have a formula for the required intensity,

$$
I \cong \frac{1}{K}\left(\frac{N h v}{\varepsilon \sum_{\text {elas }} \rho_{0}}\right)^{2 / 5}\left(F(\eta, \gamma) \Sigma_{a b s}\right)^{-3 / 5}(\Delta t)^{-11 / 5}
$$

Equation (22) shows that the required intensity will decrease as the cross sections increase and Eq. (21) shows that resolution will be improved by a lower required intensity. It is therefore to our advantage to use as large cross sections as possible, and we should use the $1 \mathrm{~S} \rightarrow \pi 2 \mathrm{P}$ resonance as in problem \#1. The intensity becomes

$$
I \cong 9.3 \times 10^{7} \mathrm{~W} \cdot \mathrm{cm}^{-2}
$$

and the linear resolution is

$$
\delta \cong 220 \AA
$$

Taking a scattering efficiency of $10^{-2}$, the recording surface exposure is about $10^{-4} \mathrm{~J} \cdot \mathrm{cm}^{-2}$, which is beyond the limit of interpretable exposure for photoresists. 
It is clear from Eqs. (21) and (22), ano from practical limits on recording surface exposures that being able to produce short pilses or shuttering the recording surface is very important to making this kind of holography a useful tool.

C. Holography with an Initially Hot Specimen

The resonant cross section is given by the Breit-Wigner formula, ${ }^{49}$ which can be written, ignoring factors on the order of unity, as

$$
\sigma \sim \frac{\lambda^{2}}{2 \pi}\left(\frac{\Gamma_{\text {res }}}{\Gamma_{\text {nat }}}\right)^{2}
$$

where $\Gamma_{\text {res }}$ is the resonant linewidth and $\Gamma_{\text {nat }}$ is the naturai linewidth. If there are no processes available to broaden the resonant linewidth beyond the natural linewidth, then $\sigma \sim \lambda^{2} / 2 \pi$. Figure 9 (page 30 ) compares $\lambda^{2} / 2 \pi$ with the cross section for photoelectric effect, coherent scattering, and incoherent scattering in nitrogen. This predicts a large cross section for coherent scattering if the resonant linewidth is nearly the same as the natural linewidth. However, the natural linewidth is usually greatly broadened by competition from other de-excitation processes, mostly Auger effect. By heating the specimen to a state in which the competition is reduced, we may be able to obtain holograms with substantially reduced $x$-ray intensity. For example, if we were able to prepare nitrogen in a He-like state, we could use the $2 \mathrm{P}$-to-1S resonance in He-like nitrogen that occurs at about $431 \mathrm{eV}$. In the He-like state, the atoms would have no Auger transitions, and therefore the resonant linewidth would closely approach the natural linewidth and the elastic cross section would be about $10^{-16} \mathrm{~cm}^{2}$. However, because a great deal of energy is required to get a substantial fraction of the atoms into the He-like state, it would be unwise to use the holography laser for heating the sample. This would only result in unnecessary exposure of the recording surface, producing an uninterpretable contrast ratio. 
TABLE IV

ABUNDANCE OF NITROGEN IONIZATION STATES

\begin{tabular}{|c|c|c|c|c|}
\hline Temp (eV) & III & IV & V & VI \\
\hline 15 & 21.9 & 53.5 & 21.1 & 1.0 \\
\hline 20 & 6.0 & 37.0 & 47.5 & 9.1 \\
\hline 25 & 1.7 & 20.1 & 55.2 & 22.9 \\
\hline 30 & .7 & 11.3 & 52.6 & 35.4 \\
\hline 35 & .25 & 6.1 & 46.4 & 47.3 \\
\hline 40 & .11 & 3.5 & 39.9 & 56.4 \\
\hline 45 & - & 2.2 & 34.3 & 63.4 \\
\hline 50 & - & 1.4 & 29.5 & 68.6 \\
\hline
\end{tabular}

To see how this works, let us again consider a concrete example. Table IV shows the abundances of the various ionization states of nitrogen as a function of temperature for a nitrogen density of $\sim 0.1 \mathrm{gm} \cdot \mathrm{cm}^{-3}$. Significant population of the He-like $\mathrm{N}^{\mathrm{VI}}$ state is found between 10 and $100 \mathrm{eV}$. We are looking for an optimum tradeoff between population of the He-like state which provides the resonant coherent scattering, and temperature, which causes hydrodynamic blurring. This optimum is near $30 \mathrm{eV}$. The heating laser could be any quantun energy for which the specimen would present a large absorption cross section; for an energy of a few $\mathrm{eV}$ it might have an absorption cross section of $10^{-16} \mathrm{~cm}^{2}$. At $30 \mathrm{eV}$ the specific energy, $i$, of our standard protein is about $3 \times 10^{13}$ $\mathrm{erg} \cdot \mathrm{gm}^{-1}$. It is not difficult to make a laser at lower quantum energies that will provide $10^{14} \mathrm{w} \cdot \mathrm{cm}^{2}$. Then the time required for the heating laser to raise standard protein to $30 \mathrm{eV}$ is $4 \times 10^{-14} \mathrm{~s}$. If the heating laser is turned off after this time, the protein will continue to expand owing to its escalated temperature. The expansion that occurs while the heating laser is on is found from Eq. (4) to be about $17 \AA$. Because nitrogen in the He-like state will not absorb radiation tuned to the coherent scattering resonance, we ignore heating by the $x$-ray laser. We assume the hydrodynanic blurring to be mostly the result of "free-run" expansion of the heated protein. Furthermore, because the protein expands only about $17 \AA$ while the heating laser is on, we will assume all the energy to be deposited at $t=0$, and calculate only the free-run expansion. 
The free-run expansion is derived in Appendix D, and from Eq. (D-37),

$$
R_{0} \cong R_{0}+\sqrt{3 f(\gamma) i} t
$$

or

$$
\Delta t \cong \frac{\delta}{\sqrt{3 f(\gamma) i}} .
$$

Because of the high temperature we can use $\gamma=5 / 3$, so if we want $\delta=100 \AA$, the exposure time must be $4 \times 10^{-3} \mathrm{~s}$. The cross section of a He-like nitrogen atom is nearly all coherent scattering. If we assume that we can then ignore heating by the $x$-ray laser, we can modify Eq. (12) to give us the intensity required to provide $10^{3}$ photons per resolution element to give

$$
I \cong \frac{\mathrm{N} \mathrm{hv}}{\varepsilon \psi \mathrm{K} \sigma_{\text {elas }} \rho \delta^{3} \Delta \mathrm{t}}=6 \times 10^{11} \mathrm{~W} \cdot \mathrm{cm}^{-2}
$$

where $\psi$ is the fraction in the He-like state (35.4\%) and we have taken $\delta=100 \AA$ and $\varepsilon=1$. This is a reasonable $x$-ray intensity, but the exposure time is very short. The exposure of the recording surface would be $\sim 10^{-1} \mathrm{~J} \cdot \mathrm{cm}^{-2}$, which is within the range of practical photoresists. ${ }^{32}$ The Doppler broadening at $30 \mathrm{eV}$ is essentially negligible, although the effect of Doppler motion on scattered coherence lengths may not be; we will discuss this in more detail later. Furthermore, in this analysis we have ignored; (1) the time dependence of the cross sections; (2) the dilation owing to $x$-ray heating, which should be small at $5 \times 10^{11} \mathrm{w} \cdot \mathrm{cm}^{2}$; and (3) the fact that Auger transitions may be present in Helike atoms in high-density plasmas. ${ }^{50}$ The task of coordinating the $x$-ray laser and the heating laser is a formidable technological challenge. 
X. THE EFFECT OF COHERENCE LENGTH AND SPECIMEN VOLUME ON RESOLUTION

A. On-Axis Fresnel Transform

It appears that the simplest technique for making hologram of microscopic biological structures is the on-axis Fresnel transform holography. The coherence length of the laser producing the hologram will affect the resolution that can be achieved in the hologram. In general, it will only be temporal coherence that degrades the resolution of the hologram because spatial coherence of indefinite extent can always be obtained by aperturing the laser and moving far enough away. In general the spatial coherence requirements are small enough so that they are expected to be a nuisance.

For any signal-to-noise threshold $(\xi)$ (the signal-to-noise ratio below $\alpha$ 'ich the fringe pattern cannot be interpreted), there is a maximum allowable distance between the specimen and the recording surface (b), which depends on the coherence length $(x)$, the wavelength $(\lambda)$, and the linear resolution sought ( $\delta)$. From Appendix $E$, this maximum distance is

$\mathrm{b} \leq \frac{(1-\xi) \mathrm{x}}{\left[1-(\lambda / \delta)^{2}\right]^{-\frac{1}{2}}-1}$

As an example, consider the case for which the fringe pattern is interpretable down to a signal-to-noise ratio of zero, $\lambda=30 \AA, \delta=200 \AA$, and $x=10 \mu \mathrm{m}$. Equation (23) gives a maximum distance between recording surface and specimen of $0.88 \mathrm{~mm}$. If the signal-to-noise threshold were $\frac{1}{2}$, the distance would be $0.44 \mathrm{~mm}$. The maximum distance requirement also places a constraint on the maximum volume of the specimen because every volume resolution element in the specimen must be able to map onto an areal resolution element on the recording surface. From Appendix D, this volume is

$$
V \leq \frac{\pi \delta \lambda^{2}(1-\xi)^{2} x^{2}}{\Delta^{2}\left(\left[1-(\lambda / \delta)^{2}\right]^{\frac{1}{2}}-1\right)^{2}}
$$


where $\Delta$ is the linear resolution of the recording surface. If $\delta \leq \lambda$, the solution of Eq. (24) is meaningless because $\delta$ is limited by diffraction. The coherence length necessary for $\delta$ to be limited by diffraction can be found from Eq. (24) to be

$$
x \geq \frac{\Delta}{1-\xi} \sqrt{\frac{V}{\pi \lambda^{3}}}
$$

If the coherence length satisfies this inequality, then coherence is not a limiting factor in resolution of the hologram.

A small prokaryotic cell (e.g., an immature bacterium E. coli.) has a volume of about $3 \times 10^{-14} \mathrm{~cm}^{3}$ while a midsize eukaryotic cell (e.g., rat liver) has a volume of about $4 \times 10^{-9} \mathrm{~cm}^{3}$. With a good photoresist (e.g., PMMA) we can obtain a recording surface resolution of about 50 to $100 \AA$. Assuming that we will be looking at nitrogen with $\lambda \sim 30 \AA$, and can interpret the fringe pattern with a signal-to-noise ratio threshold of zero, Table $V$ gives the coherence length necessary to avoid any affect on resolution of the hologram. The coherence length necessary to avoid loss of resolution goes as the inverse $3 / 2$ power of the wavelength, so increase in quantum energy can make up for broadening mechanisms. Thermal broadening of the laser frequency may produce coherence lengths between 1 and $10 \mu \mathrm{m}$ at a wavelength of $30 \AA$.

\section{TABLE V}

COHERENCE LENGTH NECESSARY TO AVOID LOSS OF RESOLUTION

Recording Resolution ( $\Delta$ )

\begin{tabular}{lccc} 
Cell Type & $50 \AA$ & $75 \AA$ & $100 \AA$ \\
\hline $\begin{array}{l}\text { Midsize eukaryote } \\
V=4 \times 10^{-9} \mathrm{~cm}^{3}\end{array}$ & $1.09 \mathrm{~mm}$ & $1.63 \mathrm{~mm}$ & $2.17 \mathrm{~mm}$ \\
$\begin{array}{c}\text { Small prokaryote } \\
V=3 \times 10^{-14} \mathrm{~cm}^{3}\end{array}$ & $2.97 \mu \mathrm{m}$ & $4.46 \mu \mathrm{m}$ & $5.95 \mu \mathrm{m}$
\end{tabular}


Equation (24) can be written as a quadratic in terms of $\delta$. Solutions to Eq. (24) for the maximum obtainable linear resolution for various coherence lengths, and recording surface resolutions are given in Table VI for a volume typical of a small prokaryotic cell and in Table VII for a volume typical of a large eukaryotic cell. Coherence length becomes a problem only for the larger cells. To obtain most flexibility it would be desirable to have a coherence length greater than $10 \mu \mathrm{m}$.

\section{B. Off-Axis Fresnel Transform}

When the reference beam impinges the recording surface at an angle different from the specimen illumination, the signal-to-noise ratio becomes a function of position on the recording surface. However, a surface average can be defined $(\xi)$ and, as shown in Appendix E, equations corresponding to Eqs. (23), (24), and (25) can be derived. It turns out that the off-axis geometry in an ideal configuration relaxes the temporal coherence requirement by a factor of $2 / \pi$, but

\section{TABLE VI}

$$
\begin{aligned}
& \text { ON-AXIS FRESNEL TRANSFORM } \\
& \text { RESOLUTION OBTAINABLE FOR SMALL PROKARYOTIC CELL } \\
& \left(\mathrm{V}=3 \times 10^{-14} \mathrm{~cm}^{3}\right)
\end{aligned}
$$

\begin{tabular}{|c|c|c|c|}
\hline $\begin{array}{l}\text { Signal-to-Noise } \\
\text { Threshold }(\xi) \\
\end{array}$ & $\begin{array}{l}\text { Recording Surface } \\
\text { Resolution }(\Delta) \\
\end{array}$ & $\begin{array}{l}\text { Coherence } \\
\text { Length (x) }\end{array}$ & $\begin{array}{c}\text { Resolution } \\
\text { Obtainable ( } \delta)\end{array}$ \\
\hline 0 & $50 \AA$ & $10 \mu \mathrm{m}$ & \multirow{2}{*}{$\begin{array}{l}\text { Diffraction } \\
\text { limited } \\
\quad 38.2\end{array}$} \\
\hline 0 & $50 \AA$ & $1 \mu \mathrm{m}$ & \\
\hline 0 & $100 \AA$ & $10 \mu \mathrm{m}$ & \multirow{2}{*}{$\begin{array}{l}\text { Diffraction } \\
\text { limited } \\
\quad 48.5 \AA\end{array}$} \\
\hline 0 & $100 \AA$ & $1 \mu \mathrm{m}$ & \\
\hline$\frac{1}{2}$ & $50 \AA$ & $10 \mu \mathrm{m}$ & \multirow{2}{*}{$\begin{array}{l}\text { Diffraction } \\
\text { limited } \\
\quad 48.5 \AA\end{array}$} \\
\hline$\frac{1}{2}$ & $50 \AA$ & $1 \mu \mathrm{m}$ & \\
\hline$\frac{1}{2}$ & $100 \AA$ & $10 \mu \mathrm{m}$ & \multirow{2}{*}{$\begin{array}{l}\text { Diffraction } \\
\text { limited } \\
\quad 62.8 \AA\end{array}$} \\
\hline$\frac{1}{2}$ & $100 \AA$ & $1 \mu \mathrm{m}$ & \\
\hline
\end{tabular}




\section{TABLE VII}

ON-AXIS FRESNEL TRANSFORM

RESOLUTION OBTAINABLE FOR TYPICAL EUKARYOTIC CELL

$$
\left(V=4 \times 10^{-9} \mathrm{~cm}^{3}\right)
$$

\begin{tabular}{r} 
Signal-t \\
Thresho \\
\hline 0 \\
0 \\
0 \\
0 \\
$\frac{1}{2}$ \\
$\frac{1}{2}$ \\
$\frac{1}{2}$ \\
$\frac{1}{2}$
\end{tabular}

\begin{tabular}{r} 
Recording \\
Resolution \\
\hline $50 \AA$ \\
$50 \AA$ \\
$100 \AA$ \\
$100 \AA$ \\
$50 \AA$ \\
$50 \AA$ \\
$100 \AA$ \\
$100 \AA$
\end{tabular}

Coherence

Length $(\mathrm{x})$

$10 \mu \mathrm{m}$

$1 \mu \mathrm{m}$

$10 \mu \mathrm{m}$

$1 \mu \mathrm{m}$

$10 \mu \mathrm{m}$

$1 \mu \mathrm{m}$

$10 \mu \mathrm{m}$

$1 \mu \mathrm{m}$
Resolution Obtainable $(\delta)$

$149 \AA$

$373 \AA$

$196 \AA$

$492 \AA$

$196 \AA$

$492 \AA$

$258 \AA$

$649 \AA$

tightens the spatial coherence requirement by a factor of $3 / 2$. The coherence lengths necessary for diffraction-limited resolution given in Table $V$ are all reduced by a factor of $2 / \pi$.

The maximum volume of the specimen as given in Eq. (24) is increased by a factor of $\pi^{2} / 4$. The linear resolution $(\delta)$ is correspondingly improved, but, because of the quartic equation, it is not improved by a simple factor. Howe' $r$, the numbers given in Tables VI and VII apply to specimens of slightly 1. yer volume. Table VI corresponds to a volume of about $7 \times 10^{-14} \mathrm{~cm}^{3}$ in the off-axis geometry and Table VII corresponds to a volume of $10^{-9} \mathrm{~cm}^{3}$.

C. Planar Fourier Transform

The optimum size lens (zone plate) for planar Fourier transform holography is about the same size as the specimen. This ensures that the reference intensity at the recording surface is about the same as the scattered intensity, thereby giving a maximum contrast ratio. The minimum temporal coherence length requirement occurs when the specimen and reference beam hole in the shadow screen are as close together as possible. This corresponds also to the minimun 
spatial coherence requirement, and is approximately twice the specimen diameter. From Appendix E, the temporal coherence requirement is

$$
x \geq \frac{a^{2}}{2 b(1-\bar{\xi})}\left[1-(\lambda / \delta)^{2}\right]^{\frac{1}{2}},
$$

where $a$ is the characteristic transverse dimension of the specimen. This is far less temporal coherence than required for the Fresnel transform techniques and varies inversely with the specimen-to-recording-surface spacing rather than directly. Repeating an example used in connection with Eq. (23), for a rat liver cell $(a \cong 15 \mu \mathrm{m})$ with $\lambda=30 \AA, \delta=200 \AA, \mathbf{b}=0.44 \mathrm{~mm}$, and $\bar{\xi}=\frac{1}{2}$, the temporal coherence need by only $0.5 \mu \mathrm{m}$. The spatial coherence need by only $30 \mu \mathrm{m}$.

Because of the inverse dependence of temporal coherence length on specimento-recording-surface spacing, the specimen volume limits resulting from coherence and mapping are separate equations. The volume limit is the lesser of the volumes given by Eqs. $(E-9)$ and $(E-10)$ in Appendix E, i.e.,

$$
V \leq \frac{\pi}{6} \operatorname{Min}\left\{[2 \operatorname{xb}(1-\xi) / \mu\}^{3 / 2}, 6 b^{2} \delta^{3}\left(\mu^{-2}-1\right) / \Delta^{2}\right\}
$$

where $\mu=\left[1-(\lambda / \delta)^{2}\right]^{\frac{1}{2}}$. The most advantageous use of Fourier transform holography is likely to be with a photocathode-microchannelplate type recording scheme (see Sec. V.C). Both $b$ and $\Delta$ will be large, so the second part of Eq. (27) will likely dominate.

\section{Spherical Fourier Transform}

In the reflector mode of spherical Fourier transform holography, the minimal spatial and temporal coherence requirements occur when the reflector and specimen are as close together as possible. Because of spherical symmetry, the dependence of temporal coherence on the diffraction angle disappears and Eq. (26) becomes 


$$
x \geq \frac{a^{2}}{2 b(1-\bar{\xi})}
$$

Unless diffraction limited resolution is being sought, both planar and spherical Fourfer schemes will have nearly the same coherence length requirements. The volume limitation corresponding to Eq. (26) is shown in Appendix E to be

$$
V \leq \frac{\pi}{6} \operatorname{Min}\left\{[2 x b(1-\bar{\xi})]^{3 / 2}, 12 b^{2} \delta^{3}(1-\mu) / \Delta^{2}\right\}
$$

and the second half will likely dominate.

\section{DOPPLER BROADENING}

The portion of the hologram that is generated by scattering from individual atoms is subject to Doppler broadening, which can destroy the phase relation with the reference beam. Thus Doppler broadening constrains the maximum distance of the specimen from the recording surface and the maximum volume of the specimen. From Appendix F, the maximum specimen volume is given by

$$
\mathrm{V} \leq \frac{3 \pi \delta \lambda^{4} c^{2} A}{8 \mathrm{~T} k^{2} \Delta^{2}\left\{1-\left[1-(\lambda / \delta)^{2}\right]^{\frac{1}{2}}\right\}} \ln ^{2}\left(\frac{\xi-\beta}{\alpha}\right)
$$

where $A$ is the atomic number of the scattering atoms, $T$ is the temperature, $c$ is the speed of light, and $k$ is a constant relating temperature and velocity. The variables in the logarithmic term are

$$
\alpha=\frac{\Sigma_{1}}{\Sigma_{1}+\Sigma_{2}} \quad \text { and } \quad \beta=\frac{\Sigma_{2}}{\Sigma_{1}+\Sigma_{2}}
$$


where $\Sigma_{1}$ is the coherent scattering cross section subject to Doppler broadening and $\Sigma_{2}$ is the coherent scattering cross section not subject to Doppler broadening. Clearly if the signal-to-noise threshold ( $(\xi)$ is less than $\beta$, there is no constraint on specimen volume imposed by Doppler broadening.

Referring to problem $\left.\right|_{1} 1$ on page 27 , we can obtain the following estimates

$$
\Sigma_{1} \cong \frac{4}{3} \pi \mathrm{K} \rho_{\mathrm{o}} \mathrm{R}_{\mathrm{o}}^{3} \sigma_{\mathrm{elas}}=3.8 \times 10^{-14} \mathrm{~cm}^{2}
$$

and

$$
\Sigma_{2} \cong \Sigma_{\text {elas }} \cong 3.4 \times 10^{-11} \mathrm{~cm}^{2}
$$

Because $\beta=0.999$, we would not expect temperature to affect our resolution unless we required $\xi>0.999$. In problem $\# 2$ on page 34 ,

$$
\Sigma_{1} \cong 4.8 \times 10^{-18} \mathrm{~cm}^{2}
$$

and

$$
\Sigma_{2} \cong 5.3 \times 10^{-16} \mathrm{~cm}^{2}
$$

so we have no constraint unless we need $\xi>0.99$. In the problem of the initially hot specimen on page 38 , we have assumed that all the coherent scattering is from individual atoms, i.e., $\Sigma_{2}=0$, and therefore $\beta=0$ and $\alpha=1$. Using $\Delta=100 \AA, \delta=100 \AA, \lambda=30 \AA, T=30 \mathrm{eV}$, and $A=14$ in Eq. (26) we find

$$
\mathrm{V} \leq 3.6 \times 10^{-11} \mathrm{ln}^{2} \xi
$$


Thus for the small prokaryote $\left(V=3 \times 10^{-14} \mathrm{~cm}^{3}\right)$ we have a relaxed signal-tonoise threshold, $\xi \leq 0.97$, but for the typical eukaryote the signal-to-noise threshold is so stringent as to make interpretable fringe patterns impossible, $\xi=2.5 \times 10^{-5}$.

Because we are unlikely to want to use an initially hot specimen anyway (the exposure time is too short), we can conclude in general that Doppler broadening is not a problem.

XII. COMPARISON OF COHERENCE REQUIREMENTS

The Fraunhofer technique appears to be more promising for near-term holography of microbiological specimens because it requires no optical components-with the possible exception of a shadow foil. However, it requires that $b \lambda \gg d^{2}$, where $d$ is the diameter of the largest feature. If the specimen cannot be described as an array of semiopaque structures in a nearly transparent cytoplasm, but looks more like shades of grey over the entire volume of the specimen, then the Fraunhofer condition must apply to the area of the entire specimen. Assuming the specimen has a near unity aspect ratio, the Fraunhofer condition imposes the following constraint on the coherence length as a function of the volume of the specimen:

$$
x \gg \frac{\left(\mu^{1}-1\right)}{(1-\xi) \lambda}\left(\frac{6}{\pi} v\right)^{2 / 3}
$$

If the specimen is a small prokaryotic cell $\left(\mathrm{V}=3 \times 10^{-14} \mathrm{~cm}^{3}\right)$ then the coherence length need only be $0.93 \mu \mathrm{m}$ for a signal-to-noise threshold of $\frac{1}{2}$. If we consider a midsized eukaryotic cell $\left(V=4 \times 10^{-9} \mathrm{~cm}^{3}\right)$, however, a coherence length of about $0.24 \mathrm{~cm}$ is required for a signal-to-noise threshold of a small E. coli bacterium could be easily imaged without interference from the virtual image in reconstruction within the coherence length we expect to be available. Rat liver cells that exhibit shades of grey, however, could not be imaged in the Fraunhofer field with the coherence lengths we expect to have available.

In the more general sense, interference between the real and virtual images will not be a problem if the coherence length satisfies the inequality: 
TABIE VIII

COMPARISON OF COHERENCE AND VOLUNE REQUIREHENTS

\begin{tabular}{|c|c|c|c|c|}
\hline $\begin{array}{l}\text { Type } \\
\qquad \text { Generic }\end{array}$ & \multicolumn{2}{|c|}{ Fresnel Transform } & \multicolumn{2}{|c|}{ Fourier Transforn } \\
\hline Specific & On-Axis & off-Axis & Planar & Spberical \\
\hline $\begin{array}{l}\text { Coherence Requirements } \\
\text { |Spatial }\end{array}$ & $2 \frac{b \lambda}{\delta \mu}$ & $3 \frac{b \lambda}{\delta \mu}$ & \multicolumn{2}{|l|}{$2 a$} \\
\hline Tempora 1 & $\frac{b}{1-\xi}\left(\mu^{-1}-1\right)$ & $\frac{2}{\pi} \frac{b}{1-\xi}\left(\mu^{-1}-1\right)$ & $\mu \frac{a^{2}}{2 b(1-\xi)}$ & $\frac{a^{2}}{2 b(1-\xi)}$ \\
\hline $\begin{array}{l}\text { Maximum Specimen Volume } \\
\qquad \begin{array}{l}\text { Coherence } \\
\text { Limit }\end{array}\end{array}$ & \multirow{2}{*}{$\pi \frac{\delta \lambda^{2}(1-\xi)^{2} x^{2}}{\Delta^{2}(\mu-1)^{2}}$} & \multirow{2}{*}{$\frac{\pi^{3}}{4} \frac{\delta \lambda^{2}(1-\xi)^{2} x^{3}}{\Delta^{2}(\mu-1)}$} & $\mu^{-3 / 2} \frac{\pi}{6}[2 \times b(1-\xi)]^{3 / 2}$ & $\frac{\pi}{6}[2 \times b(1-\xi)]^{3 / 2}$ \\
\hline $\begin{array}{l}\text { Mapping } \\
\text { Limit }\end{array}$ & & & $\left(\mu^{-2}-1\right) \frac{\pi b^{2} \delta^{3}}{\Delta^{2}}$ & $2(1-\mu) \frac{\pi b^{2} \delta^{3}}{\Delta^{2}}$ \\
\hline $\begin{array}{l}\text { Doppler } \\
\text { Limit }\end{array}$ & \multicolumn{3}{|c|}{$\frac{3 \pi \delta \lambda^{4} c^{2} A \ln ^{2} \xi}{8 I k^{2} \Delta^{2}(1-\mu)}$} & $\frac{3 \pi \delta \lambda^{4} c^{2} A \ln ^{2} \xi}{8 T k^{2} \Delta^{2} \mu^{2}(1-\mu)}$ \\
\hline
\end{tabular}

$x \gg \frac{\Delta^{4} \mu^{3}(\mu-1)}{36 \lambda \delta^{2}(1-\xi)^{3}}$

which is derived from Eq. (24).

Table VIII compares the spatial and temporal coherence length requirements and the maximum specimen volumes for the four basic holographic techniques we have considered.

XIII. CONCLUSIONS

It appears that it is most practical to use Fresnel transform holography with a photoresist recording surface. The recording properties of photoresist are optimum in the wavelength region where we could use resonance scattering from nitrogen. To achieve a good contrast ratio we nay need to use a "shadow foil" to attenuate the reference beam. The shadow foil must be physically homogeneous on the scale of $x$-ray wavelengths so that it does not significantly alter the temporal or spatial coherence of the reference bean. Spherical Fourier transform holography may be a reasonable alternative, particularly since there seem to be ways to use the reflector as a shutter nechanisn, or to use an 
array of gated microchannelplates as the recording medium. But the technological developments that would be required for the realization of spherical Fourier transform holography are formidable, and the problems attending the preparation of a shadow foil are considerably less stressing than those of making and measuring a suitable reflector.

From our discussion of hydrodynamic blurring, and the formulas that we have derived relating resolution to intensity and exposure time, we find that for 100- $\AA$ resolution we need intensities of $10^{11}$ to $10^{12} \mathrm{~W} \cdot \mathrm{cm}^{-2}$ and pulse lengths of only a few picoseconds. While this is not a particularly difficult intensity range to achieve, the exposure times are disturbingly short. We therefore should carefully consider potential technologies that might provide means for gating the recording surface or shuttering the laser beam. If the recording surface is sufficiently sensitive, we can tolerate longer laser pulses with lower intensity. In the long run, however, it would be more fruitful to develop technologies for reducing the exposure time than it would be to find more sensitive recording media.

$\mathrm{X}$-ray holography will provide a means for imaging the density distribution of individual elements, or in some cases particular chemical bonds with these elements. Nitrogen, phosphorous, sulfur, and calcium are all interesting candidates for biological reasons. But, it seems that it will be most scientifically profitable to image nitrogen at an early stage of $x$-ray holographic investigations because: (1) nitrogen is found in highest density in the proteins, and proteins generally constitute the structural building blocks of cells; (2) it is of reasonable high abundance; and (3) its K-edge, where the most pronounced resonances will be found, is at an energy of only 400 volts. Because of my special interest in nitrogen, this paper has been somewhat specialized to that element, but all of the principles and quantitative relationships derived here would apply equally well to concentrations of these other elements.

The relations derived in this paper indicate that higher resolution can be obtained with larger $x$-ray cross sections. To the extent that the total scattering cross section of an individual biological feature is more dependent on the extinction of the $x$-ray beam through that feature than it is on the coherence scattering cross sections of the individual atoms within that feature, it is more important to have a large total atomic cross section than a large ratio of elastic to absorption components of that cross section. It therefore seems particularly attractive to use $x$-ray resonances to obtain large cross sections. 
In this paper we have called attention to the $1 \mathrm{~S} \rightarrow 2 \pi \mathrm{P}$ cross section that occurs in molecular nitrogen. This resonance involves elevating a $\mathrm{K}$-shell electron from a nitrogen atom to a vacant antibonding orbital in the molecule. Analogous orbitals probably exist in the proteins, and it would be very profitable to investigate, measure, and characterize the resulting resonances.

When added to the armamentarium of the research biologist, high-intensity $x$-ray holography may offer: (1) the ability to make high-resolution three-dimensional pictures of living cells; (2) the ability to distinguish individual atomic species; and (3) the ability to time resolve activities within a cell to picosecond scale. However, this technology is so radically new and so entirely different from anything that has been available to the biological sciences in the past, that it is impossible to predict in what way it will ultimately be most beneficial.

\section{APPENDIX A}

CONSIDERATIONS IN SELECTING SPECIMENS AND WAVELENGTH

\section{SPECIMEN TYPES}

A significant advantage offered by the use of a high-intensity $x$-ray laser is the ability to image biological specimens in the living state. Most biologists distinguish among three elementary life units, which in descending order of size are: (1) eukaryotic cells, (2) prokaryotic cells, and (3) viruses.

A eukaryote is a cell with a well-defined nucleus. It is the type of cell of which most higher plants and animals are composed. Many one-celled organisms, protozoa, are eukaryotic cells as well as fungi and most algae. Eukaryotes have a membrane surrounding the outer extent of their protoplasm, a membrane surrounding the nucleus, and membranes surrounding various organelles within the cell. Eukaryotes are the most recent cell to evolve, and many of their metabolic functions are compartmentalized. As shown in Table I (page 7), eukaryotes are mostly water. Their structural elements are mostly composed of protein. 
Other prominent constituents are the nucleic acids, the lipids, the carbohydrates, and the salts.

Prokaryotic cells are distinguished from eukaryotic cells by the lack of a well-defined nucleus. In general, they are much smaller than eukaryotes, their volumes being $10^{-3}$ to $10^{-4}$ as large. Their composition differs from eukaryotes most conspicuously by the larger abundance of nucleic acids. This may range from $1 \%$ DNA and 6\% RNA for Escherichia coli to 4\% DNA and $8 \%$ RNA for Mycoplasma gallisepticum. The mycoplasma are extremely small cells that lack a true cell wall.

Viruses are particles of nucleic acid surrounded by specific proteins. Many biologists consider them to be only on the thrishold of life since they have no metabolism in the usual sense, and must use the biosynthetic machinery of a host cell to accomplish their replication. The nucleic acid component of viruses may be either RNA or DNA but not both.

II. STANDARD COMPOSITIONS FOR BIOLOGICAL CONSTITUENTS

For the purpose of calculations $I$ wish to establish a standard chemical composition for each of the constituents shown in Table $I$.

Water: Whether chemically bound or free, water is $\mathrm{H}_{2} \mathrm{O}$.

Protein: Proteins are very large molecules that may serve a multitude of functions. They are polymers of nitrogen-bearing amino acids and may range in molecular weight from $10^{4}$ to $10^{7}$ daltons. Their main importance to holography is that they are the main constituent of most of

TABLE A-I

ELEMENTARY COMPOSITION OF PROTEIN

Hydrogen

Carbon

Oxygen

Nitrogen

Sulfur

\begin{tabular}{rr}
\multicolumn{1}{c}{ Mass } & \multicolumn{1}{c}{ Atonic } \\
$6-8 \%$ & $43-58 \%$ \\
$45-55 \%$ & $27-33 \%$ \\
$19-25 \%$ & $9-11 \%$ \\
$14-20 \%$ & $7-10 \%$ \\
$0-4 \%$ & $0-1 \%$
\end{tabular}


the structure elements of a cell, although carbohydrates may contribute in plant cells. Their elementary makeup is given in Table A-I. An average of atomic abundances leads to the somewhat arbitrary composition $\mathrm{H}_{50} \mathrm{C}_{30} \mathrm{~N}_{9} \mathrm{O}_{10} \mathrm{~S}_{1}$.

Nucleic Acids: Deoxyribonucleic acid (DNA) and ribonucleic acid (RNA) are enormous molecules containing genetic information that is transiated into the synthesis of proteins. Each is a polymer consisting of a repeated covalent backbone binding a series of bases, the sequence of which is the genetic code. The composition of the covalent backbone for DNA is $\mathrm{H}_{8} \mathrm{C}_{5} \mathrm{O}_{5} \mathrm{P}_{1}$, and for RNA is $\mathrm{H}_{8} \mathrm{C}_{5} \mathrm{O}_{6} \mathrm{P}_{1}$. The possible bases consist of two purines (adenine and guanine) and three pyrimidines (uracil, thymine, and cytosine). Adenine, guanine, and cytosine are bases for both DNA and RNA. The fourth base for DNA is thymine, and the fourth base for RNA is uracil. The relative amounts of the four bases for DNA and RNA vary from organism to organism. It is a good approximation, however, to say that approximately equal quantities of the four bases appear in each organism. For the purpose of establishing a standard, I take equal quantities of the four bases for both RNA and DNA. The process of arriving at this standard is summarized in Table A-II.

Lipids: The lipids are comprised of a wide variety of chemical structures all sharing common solvents. They encompass: (1) fat, a triglyceride ester of glycerol and three fatty acid molecules; (2) phospholipids, complex organic compounds containing phosphorous; (3) waxes, esters of higher allophitic alcohols and higher fatty acids distinguished by longer chain lengths than fatty acids of other lipid classes; (4) sterols, aromatic alcohols with a free hydroxyl group; and (5) carotenoids, a group of red and yellow pigments with a multitude of functions. Since the lipids are a very complex group of compounds, it is difficult to characterize them in general. For the purpose of establishing a standard composition, I take a rough average of $\mathrm{H}_{10} \mathrm{C}_{5} \mathrm{O}_{1}$. Even though both phosphorous and nitrogen appear in the phospholipids, the quantity on average is relatively so small that it can be ignored. 


\section{Purines}

$$
\begin{array}{ll}
\text { Adenine (A) : } & \mathrm{H}_{5} \mathrm{C}_{5} \mathrm{~N}_{5} \\
\text { Guanine (G) : } & \mathrm{H}_{5} \mathrm{C}_{5} \mathrm{~N}_{5} \mathrm{O}_{1}
\end{array}
$$

Pyrimidines

$$
\begin{array}{ll}
\text { Uracil (U) : } & \mathrm{H}_{4} \mathrm{C}_{4} \mathrm{~N}_{2} \mathrm{O}_{2} \\
\text { Thymine (T) : } & \mathrm{H}_{6} \mathrm{C}_{5} \mathrm{~N}_{2} \mathrm{O}_{2} \\
\text { Cytosine (C): } & \mathrm{H}_{5} \mathrm{C}_{4} \mathrm{~N}_{3} \mathrm{O}_{1}
\end{array}
$$

Covalent Backbone

$$
\begin{array}{ll}
\text { DNA } & : \mathrm{H}_{8} \mathrm{C}_{5} \mathrm{O}_{5} \mathrm{P}_{1} \\
\text { RNA } & : \mathrm{H}_{8} \mathrm{C}_{5} \mathrm{O}_{6} \mathrm{P}_{1}
\end{array}
$$

Standard Composition

$$
\begin{aligned}
\text { DNA } & \cong 4 \times \text { Backbone }+\mathrm{A}+\mathrm{G}+\mathrm{T}+\mathrm{C} \\
& =\mathrm{H}_{53} \mathrm{C}_{39^{\mathrm{N}} 15^{\mathrm{O}}{ }_{24} \mathrm{P}} \\
\text { RNA } & \cong 4 \times \text { Backbone }+\mathrm{A}+\mathrm{G}+\mathrm{U}+\mathrm{C} \\
& =\mathrm{H}_{51} \mathrm{C}_{38^{N}} \mathrm{~N}_{15}{ }^{\mathrm{O}}{ }_{28} \mathrm{P}_{4}
\end{aligned}
$$

TABLE A-III

STANDARD COMPOSITIONS OF MOST ABUNDANT BIOLOGICAL CONSTITUENTS

Constituent

Water

Protein

DNA

RNA

Lipid

Carbohydrate
Standard

Composition

$\mathrm{H}_{2} \mathrm{O}$

$\mathrm{H}_{50} \mathrm{C}_{30} \mathrm{~N}_{9} \mathrm{O}_{10} \mathrm{~S}_{1}$

$\mathrm{H}_{53} \mathrm{C}_{39} \mathrm{~N}_{15} \mathrm{O}_{24} \mathrm{P}_{4}$

$\mathrm{H}_{51} \mathrm{C}_{38} \mathrm{~N}_{15}{ }^{\mathrm{O}_{28}} \mathrm{P}_{4}$

$\mathrm{H}_{10} \mathrm{C}_{5}{ }^{0}{ }_{1}$

$\mathrm{H}_{2} \mathrm{C}_{1} \mathrm{O}_{1}$ 
Carbohydrates: By definition, carbohydrates are compounds that contain hydrogen, carbon, and oxygen in the ratios of $2: 1: 1$, hence I take the composition $\mathrm{H}_{2} \mathrm{C}_{1} \mathrm{O}_{1}$.

Salts: These inorganic compounds are of such low abundance and such variation in chemical composition that I will ignore them for the purpose of describing $x$-ray properties.

Table A-III summarizes the standard composition of the most abundant biological constituents.

\section{THE SPECIAL PROPERTIES OF NITROGEN}

Figure 9 (page 30) compares the cross sections for photoelectric effect, coherent scattering, and incoherent scattering with the quantity $\lambda^{2} / 2 \pi$, which is on the order of the resonant cross section as given by the Breit-Wigner formu$1 \mathrm{a}^{44}$ when the resonant linewidth is close to the natural linewidth. An example of a resonance that could be expected to approach this idealized value is the $1 \mathrm{P} \rightarrow 1 \mathrm{~S}$ transition in helium-like nitrogen that occurs at about $431 \mathrm{eV}$.

Figure $A-1$ shows the $x$-ray cross section of molecular nitrogen in the range from 400-435 eV as reported by Bianconi et a1.51 A resonance occurs at about $401.3 \mathrm{eV}$ owing to the promotion of a $1 \mathrm{~S}$ electron from the first unfilled $\left(\pi_{\mathrm{g}} 2 \mathrm{P}\right)$ orbital. 52 While the total cross section of this resonance is about $17.4 \mathrm{Mb}$, its fluorescent yield (the fraction of its cross section that de-excites by radiative processes), is only about 1 part in $10^{3}$. The coherent scattering cross section is even smaller. This resonance is for $\mathrm{N}_{2}$ but $I$ would expect that a similar resonance might be encountered for nitrogen in the polypeptide chains that comprise the proteins. If a first vacant orbital exists in the polypeptide bond, the corresponding rasonance will differ from the one in $\mathrm{N}_{2}$ only by the difference in $\mathrm{K}$-shell ionization potential, which depends on the molecular configuration. Bianconi et al.51 showed that corresponding resonances exist in molecular nitrous oxide $\left(\mathrm{N}_{2} \mathrm{O}\right)$. These were found at $401.2 \mathrm{eV}$ and $404.9 \mathrm{eV}$, corresponding to the ionization potential of the "terminal" nitrogen, and the "centra1" nitrogen. The ratio of the height of each of the $\mathrm{N}_{2} \mathrm{O}$ resonances to the corresponding nitrogen K-edge is about the same as the height of the $\mathrm{N}_{2}$ resonance to its corresponding nitrogen K-edge. For the purpose of computation, I will assume that if the resonance exists in the protein polypeptide chain and in the bonds of the purines and pyrimidines in nucleic acid, the corresponding 
cross section will be about $17.4 \mathrm{Mb}$, i.e., the same as Bianconi et al. observed for molecular nitrogen. Use of the nitrogen resonance for holography is attractive not only because of the enormity of the expected cross section in both protein and nucleic acid, but also because the $x$-ray absorption coefficient for water is nearer a local minimum at this energy.

IV. CONTRAST RATIO FOR BIOLOGICAL CONSTITUENTS

Table A-IV shows the $x$-ray absorption lengths for the most abun-

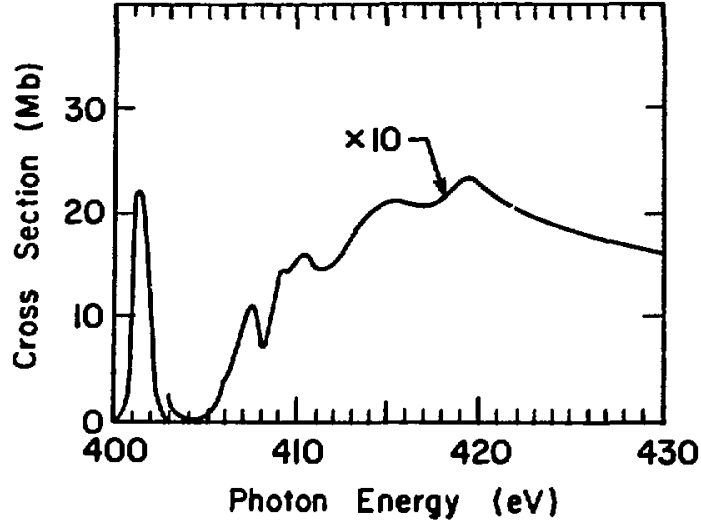

Fig. A-1. K-shell absorption spectrum of $\mathrm{N}_{2}$ as determined photoelectrically by Bianconi et al. Notice enormous resonance at $401.3 \mathrm{eV}$ for the $1 \mathrm{~S} \rightarrow \pi 2 \mathrm{P}$ transition. (Adapted from figure in Ref. 51). dant biological constituents. The $x$ ray absorption length is the distance over which the $x$-ray flux diminishes by a factor of $1 / e$, and is the reciprocal of the $x$-ray absorption coefficient. Each

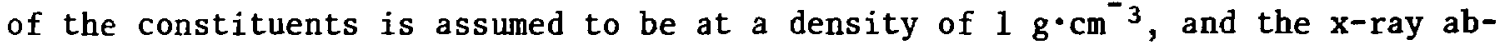
sorption length is measured in $\AA$. For $x$-rays not tuned to a resonance, the greatest constrast ratio between water, which comprises mo of the cytoplasm, and protein or the nucleic acids, occurs at a quantum energy of about $400 \mathrm{eV}$. In fact, the absorption length for water is nearly an order of magnitude greater than the absorption length for any of the other constituents. The reason for the high contrast is that the cross section for oxygen is near a minimum. If the $x$-ray energy is tuned to a nitrogen resonance, which will be in the vicinity of $400 \mathrm{eV}$, then the contrast ratio between water and protein is nearly two orders of magnitude and similarly for the contrast ratio between water and the nucleic acids. Thus when the nitrogen resonance is utilized, structural elements containing protein, and the chromosomes consisting of long strands of DNA wound on histones, will all appear black compared to the cytoplasm which will be essentially transparent. This is the optimum arrangement for several kinds of holography. 
TABLE A-IV

X-RAY ABSORPTION LENGTHS FOR MOST ABUNDANT BIOLOGICAL CONSTITUENTS

Constituent

X-ray Absorption Length \&

\begin{tabular}{|c|c|c|c|c|c|}
\hline & $200 \mathrm{eV}$ & $400 \mathrm{ev}$ & $800 \mathrm{eV}$ & $1600 \mathrm{eV}$ & Resonance \\
\hline Water & $8.45 \times 10^{3}$ & $4.69 \times 10^{4}$ & $1.34 \times 10^{4}$ & $8.72 \times 10^{4}$ & $4.69 \times 10^{4}$ \\
\hline Protein & $9.31 \times 10^{3}$ & $6.75 \times 10^{3}$ & $1.96 \times 10^{4}$ & $1.34 \times 10^{5}$ & $6.93 \times 10^{2}$ \\
\hline DNA & $6.31 \times 10^{3}$ & $7.83 \times 10^{3}$ & $1.82 \times 10^{4}$ & $1.22 \times 10^{5}$ & $7.20 \times 10^{2}$ \\
\hline RHA & $6.31 \times 10^{3}$ & $8.23 \times 10^{3}$ & $1.77 \times 10^{4}$ & $1.18 \times 10^{5}$ & $7.58 \times 10^{2}$ \\
\hline Lipid & $1.58 \times 10^{4}$ & $5.34 \times 10^{3}$ & $2.28 \times 10^{4}$ & $1.57 \times 10^{5}$ & $5.34 \times 10^{3}$ \\
\hline Carbohydrate & $1.08 \times 10^{4}$ & $8.51 \times 10^{3}$ & $1.64 \times 10^{4}$ & $1.09 \times 10^{5}$ & $8.51 \times 10^{3}$ \\
\hline
\end{tabular}

V. ELIGIBILITY OF VARIOUS BIOLOGICAL SPECIMENS FOR X-RAY HOLOGRAPHY

To be a good candidate for $x$-ray holographic investigation, a specimen must not only display a large contrast ratio between the features $I$ am seeking to image, but must also be reasonably transparent to the $x$ rays $I$ am using to generate the hologram. Table A-V lists examples of biological specimens that might be candidates for $x$-ray holography, ranging over 13 orders of magnitude in total mass. Table A-V lists the greatest dimension of the specinen, a characteristic dimension that might be traversed by the $x$-ray flux if the specimens were randomly oriented, and the $x$-ray transmission through the specimen for various quantum energies, including that for the nitrogen resonance in both protein and the nucleic acids. The $x$-ray transmission is calculated as if the specimen were homogenized, that is, if all the constituents of the specimen were evenly distributed. In fact, the transmission will always be sonewhat greater than the numbers shown because the various constituents of the specimen will be localized. In calculating the entries in this table, actual compositions of the listed specimens or best estimates thereto have been used in each case. Within each mass category there certainly exists certain specinen with greater and less $x$-ray transmission than the specific specimen indicated.

The table suggests that the largest specinen for which I could expect sufficient transmission for an efficient hologran has a mass of about $10^{-8} \mathrm{~g}$. The x-ray transmission at $1600 \mathrm{eV}$ is acceptable for specimens of mass up to $10^{-7} \mathrm{~g}$, however, as shown by Table $A-V$, contrast ratio at this quantum energy is 
TABLE A-V

MASS, DIMENSIONS, AND X-RAY TRANSMISSION OF VARIOUS BIOLOGICAL SPECIMENS

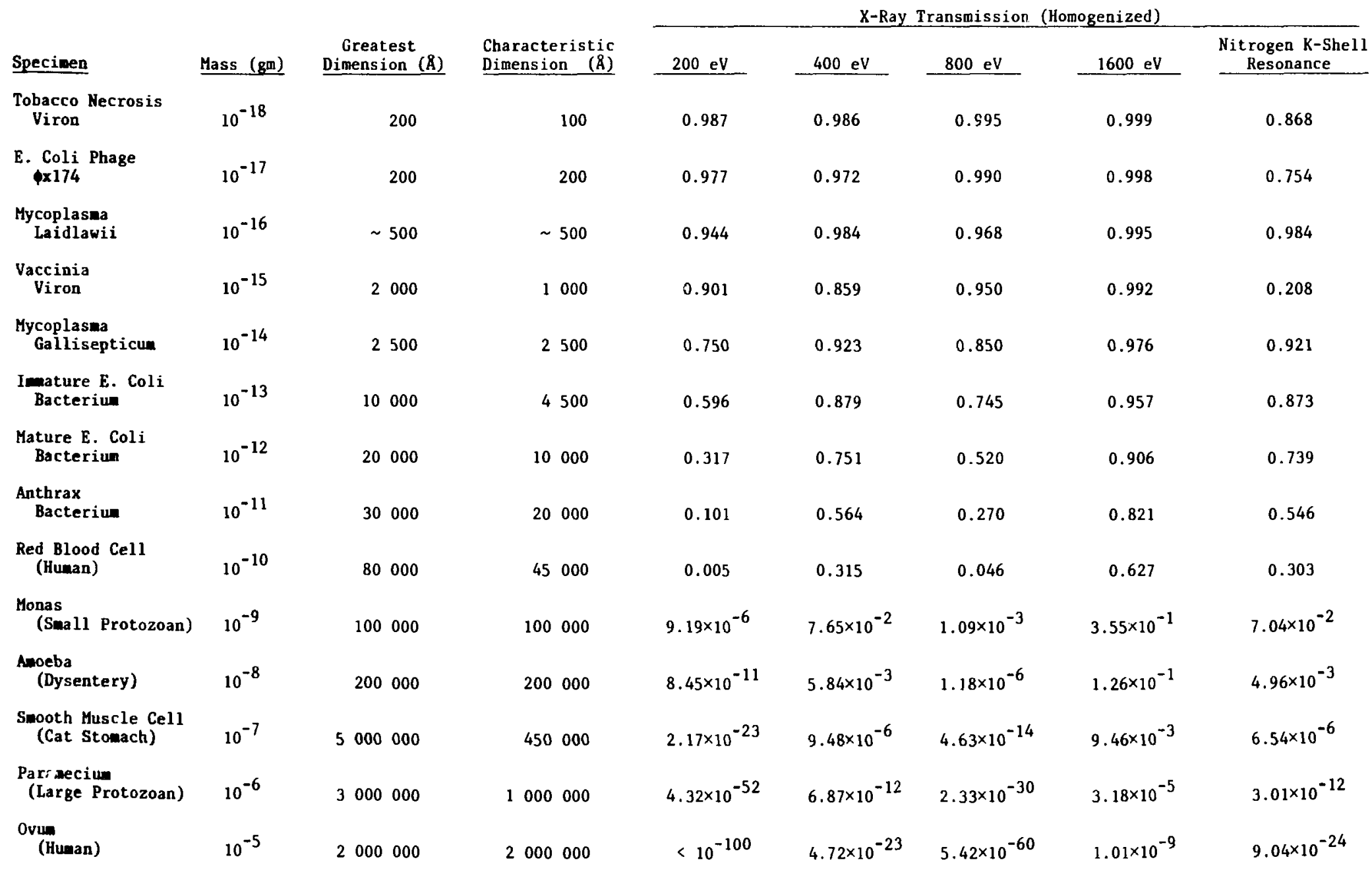


not good as, and constructing an $x$-ray laser of this quantum energy is considered very difficult. (The highest quantum energy available for neon-like lasers, neon-like uranium, is limited to about $1200 \mathrm{eV}$.) The larger specimens áre generally not symmetrical and can be aligned and even squeezed a bit to reduce the total distance through the specimen that the $x$ rays must travel. This can be accomplished by squeezing the specimen between the front and rear windows of the specimen holder. The smallest dimension shown in Table VII reflects the limit to which the specimen can be squeezed when properly oriented, and still maintain its normal metabolism and other biological functions. Aligning or squeezing the specimen mitigates the transmission problem, and as illustrated by Table A-VI, allows living cells as massive as $10^{-7} \mathrm{~g}$ to be easily holographed at $400 \mathrm{eV}$, or by using the nitrogen $\mathrm{K}$-shell resonance.

TABLE A-VI

X-RAY TRANSHISSION OF "SQUEEZED" CELLS

\begin{tabular}{|c|c|c|c|c|c|c|c|}
\hline \multirow[b]{2}{*}{ Specimen } & \multirow[b]{2}{*}{ Mass (gD) } & \multirow[b]{2}{*}{$\begin{array}{c}\begin{array}{c}\text { Smallest } \\
\text { Dimension }(\AA)\end{array} \\
\end{array}$} & \multicolumn{5}{|c|}{ X-Ray Transmission (Homogenized) } \\
\hline & & & $200 \mathrm{eV}$ & $400 \mathrm{eV}$ & $800 \mathrm{eV}$ & $1600 \mathrm{eV}$ & $\begin{array}{l}\text { Nitrogen k-shell } \\
\text { Rescnance } \\
\end{array}$ \\
\hline $\begin{array}{l}\text { Red Blood Cell } \\
\text { (Human) }\end{array}$ & $10^{-10}$ & $3 \times 10^{4}$ & $3.08 \times 10^{-2}$ & $4.62 \times 10^{-1}$ & $1.29 \times 10^{-1}$ & $7.33 \times 10^{-1}$ & $.51 \times 10^{-1}$ \\
\hline $\begin{array}{l}\text { Monas } \\
\text { (Smali) }\end{array}$ & $10^{-9}$ & $5 \times 10^{4}$ & $3.03 \times 10^{-3}$ & $2.77 \times 10^{-1}$ & $3.30 \times 10^{-2}$ & $5.96 \times 10^{-1}$ & $2.65 \times 10^{-1}$ \\
\hline $\begin{array}{l}\text { Amaeba } \\
\text { (Dysentery) }\end{array}$ & $10^{-8}$ & $2 \times 10^{4}$ & $9.83 \times 10^{-2}$ & $5.98 \times 10^{-1}$ & $2.55 \times 10^{-1}$ & $8.13 \times 10^{-1}$ & $5.88 \times 10^{-1}$ \\
\hline $\begin{array}{l}\text { Smooth Muscle Cell } \\
\text { (Cat Stomach) }\end{array}$ & $10^{-7}$ & $1 \times 10^{5}$ & $9.19 \times 10^{-6}$ & $7.65 \times 10^{-2}$ & $1.05 \times 10^{-3}$ & $3.55 \times 10^{-1}$ & $7.04 \times 10^{-2}$ \\
\hline $\begin{array}{l}\text { Paramecium } \\
\text { (Large) }\end{array}$ & $10^{-6}$ & $6 \times 10^{5}$ & $6.04 \times 10^{-31}$ & $2.01 \times 10^{-7}$ & $1.66 \times 10^{-18}$ & $2.00 \times 10^{-3}$ & $1.22 \times 10^{-7}$ \\
\hline
\end{tabular}




\section{APPENDIX B}

\section{GRAINSIZE INDEPENDENT HOLOGRAPHY}

\section{PLANAR FOURIER TRANSFORM HOLOGRAPHY}

To see how the Fourier transform technique can be used to obtain an interpretable hologram with resolution independent of grain size in the recording medium, consider, in rectangular coordinates a reference source emitting spherical waves from position $(0,0,0)$, and a specimen comprised of two similar spherical wave enitters at positions, $(0, c, 0)$ and $(0, d, 0)$.

The amplitude from the three sources at any point in space $(x, y, z)$ is given by the following:

$$
\begin{array}{ll}
\text { Reference: } & a_{r}=\frac{A}{\sqrt{x^{2}+y^{2}+b^{2}}} \exp \left\{-i k \sqrt{x^{2}+y^{2}+b^{2}}\right\} \\
\text { Specimen Point 1: } & a_{s 1}=\frac{a}{\sqrt{x^{2}+(y-c)^{2}+b^{2}}} \exp \left\{-i k \sqrt{x^{2}+(y-c)^{2}+b^{2}}\right\} \\
\text { Specimen Point 2: } & a_{s 2}=\frac{a}{\sqrt{x^{2}+(y-d)^{2}+b^{2}}} \exp \left\{-i k \sqrt{x^{2}+(y-d)^{2}+b^{2}}\right\} .
\end{array}
$$

Now if both $c$ and $d$ are much snaller than $b$, then

$$
\sqrt{x^{2}+(y-c)^{2}+b^{2}} \cong r \sqrt{1-2 c \frac{y}{r^{2}}} \cong r\left(1-\frac{c y}{r^{2}}\right)
$$

where $r^{2}=x^{2}+y^{2}+z^{2}$, and similarly for the radical in the amplitude for specimen point 2 .

If the recording surface is a plane parallel to the $y$-axis, the total amplitude at that plane is 


$$
\begin{aligned}
\stackrel{a}{n}_{n} & =\underline{a}_{r}+\underline{a}_{s}+\underline{a}_{s} 2 \\
& =\frac{1}{r} \exp \{-i k r\}\left[A+a \exp \left\{-i k c \frac{y}{r}\right\}+a \exp \left\{-i k d{\underset{r}{r}}_{\}}\right],\right.
\end{aligned}
$$

and the intensity is

$$
\begin{aligned}
I & =\underline{a}_{n} \stackrel{a}{*}_{n}^{*} \\
& =\frac{1}{r^{2}}\left[A^{2}+2 a^{2}+2 A a \cos \left(\frac{k c y}{r}\right)+2 A a \cos \left(\frac{k d y}{r}\right)+2 a^{2} \cos \left(\frac{k(c-d) y}{r}\right)\right] \cos \theta .
\end{aligned}
$$

where $\theta$ is the angle between the vector $\vec{r}$ and the recording surface. If we consider only the line on the recording surface for which $x=0$, then $\cos \theta=$ $\sqrt{1-\left(y^{2} / r^{2}\right)}$. Now, if $A \gg a$, then

$$
\begin{aligned}
I & \cong \frac{1}{r^{2}}\left[A^{2}+2 A a\left(\cos \frac{k c y}{r}+\cos \frac{k d y}{r}\right)\right] \cos \theta \\
& \cong \frac{A^{2}}{r^{2}}\left(1+\frac{4 a}{A} \cdot \cos \frac{k(c+d) y}{2 r} \cdot \cos \frac{k(c-d) y}{2 r}\right) \cos \theta \\
& \cong \frac{A^{2}}{r^{2}}\left(1+\frac{4 a}{A} \cdot \cos \frac{\pi(c+d) y}{2 \lambda r} \cdot \cos \frac{\pi(c-d) y}{2 \lambda r}\right) \cos \theta .
\end{aligned}
$$

Any fringe spacing can be obtained by adjusting the value of $r$. Therefore grain size is not a problem, and can be compensated at the sacrifice of intensity. The pattern [Eq. (B-3)] is a DC level plus a rapidly oscillating function with spatial frequency proportional to distance from the specimen centroid to the reference source, modulated at a spatial frequency proportional to the distance between the specimen points. Equations (B-1) consider the reference and specimen points to be colinear, but similar results are obtain if they are in a random pattern. It is clear from the linearity of the amplitude equations that similar results will be obtained for specimens consisting of 3,4 , and $n$ points. 
II. SPHERICAL FOURIER TRANSFORM HOLOGRAPHY

If we use a spherical shell as the recording surface, we can eliminate the problems that occur at large diffraction angles, specifically, the loss of intensity owing to large values of $r$ at angles approaching 2 , and the noise owing to recording surface roughness, which becomes more important at grazing angles. With the reference source at the origin $(0,0,0)$ and the specimen points along the $y$-axis at $(0, c, 0)$ and $(0, d, 0)$ as before, the amplitudes in spherical coordinates are given by

$$
\begin{array}{ll}
\text { Reference: } & \underline{a}_{r}=\frac{A}{r} \exp \{-i k r\} \\
\text { Specimen Point 1: } & \stackrel{a}{s} 1 \cong \frac{a}{r}\{-i k(r-c \sin \theta \sin \phi\} \\
\text { Specimen Point 2: } & \quad{ }_{-s} \cong \frac{a}{r}\{-i k(r-d \sin \theta \sin \phi)\} .
\end{array}
$$

By inspection it is clear that the sum $\stackrel{a}{a}_{n}=\underline{a}_{r}+\underline{a}_{s 1}+\underline{a}_{s 2}$ will result in an equation analogous to Eq. (B-2) and if $\mathrm{A} \gg$ a, the equation analogous to Eq. (B-3) will be

$$
I=A^{2}\left[1+\frac{4 a}{A} \cdot \cos \frac{\pi(c+d) \sin \theta \cos \phi}{\lambda} \cdot \cos \frac{\pi(c-d) \sin \theta \cos \phi}{\lambda}\right] .
$$

Equation (B-5) gives the intensity on a unit spherical shell, and is a functirn of the usual angular and azmuthal coordinates $\theta$ and $\phi$. The spacing of fringes on the shell can be adjusted to any size by varying the radius of the shell. It has the same properties in angular coordinates as Eq. (B-3) has in spatial coordinates. Clearly the fundamental properties of grain-size independence remain the same regardless of how many points make up the specimen, as more points add only more equations of the same form to Eq. (B-4). 
III. THEOREM ON RESOLUTION OF FOURIER TRANSFORM HOLOGRAPHY USING A FRESNEL ZONE PLATE TO GENERATE THE REFERENCE SOURCE

Fourier holography requires spherically diverging reference waves. Normally this is thought of a point source producing a spherical wave front. If our source is a laser for both illumination and reference waves, we must convert plane waves to spherical waves. This is done with a lens as shown in Fig. 7, or with a Fresnel zone plate in the case of x-rays.

The divergence angle produced by a Fresnel zone plate is

$$
\theta=\sin ^{-1}(\lambda / a)
$$

where $a$ is the finest zone-plate ring spacing and $\lambda$ is the wavelength. The modulation produced by specimen point spacing is found from Eq. (B-3) to be

$$
\cos \frac{\pi(c-d)}{2 \lambda r}
$$

The low-frequency cutoff on the modulation is given by

$$
\frac{c-d}{2 \lambda r} \text { e } \geq 1
$$

where $e$ is the radius of the recording surface are illuminated by the reference source. Equation (B-7) places a limit on resolution because it limits the difference (c-d). From Eq. (B-6)

$$
e=r \sin \theta=\frac{r \lambda}{a},
$$

and from Eq. (B-7) 
or the resolution of the Fourier hologram is limited to twice the smallest spacing on the Fresnel zone plate.

APPENDIX C

IMAGE BLURRING BY A LENSLESS ELECTRON MICROSCOPE

A lensless electron microscope could consist of two concentric spherical shells of radii $r_{2}>r_{1}$. The inner shell would be a photocathode on which the hologram was registered, and the outer shell would be a recording mediun for the enlarged hologram image (a fluor, electron sensitive film, or form of on-line readout). The magnification would be simply $r_{2} / r_{1}$. If the potentials on the inner and outer shells are respectively $V_{1}$ and $V_{2}$ then the potential between the she11s is

$$
V(r)=\frac{\left(v_{1}-v_{2}\right) \frac{r_{1} r_{2}}{r}+v_{2} r_{2}-v_{1} r_{1}}{r_{1} r_{2}}
$$

For simplicity we ground the outer she11 $\left(V_{2}=0\right)$. Then Eq. $(C-1)$ becomes

$$
v(r)=v_{0}\left(\frac{r_{2}}{r}-1\right),
$$

where 


$$
V_{0}=\frac{v_{1} r_{1}}{r_{1}-r_{2}}
$$

Now an electron emerges from the photocathode with kinetic energy $E_{0}$, which may be the difference between the energy of photoemission and the work function. This initial velocity has radial and tangential components, so

$$
E_{0}=\frac{1}{2} m\left(v_{t}^{2}+v_{r}^{2}\right)
$$

where $\mathrm{m}$ is the electron mass. The angular velocity is

$$
\begin{aligned}
\dot{\theta} & =\frac{\mathrm{L}}{\mathrm{m} \mathrm{r}^{2}} \\
& =\frac{\mathrm{v}_{\mathrm{t}} \mathrm{r}_{1}}{\mathrm{r}^{2}} .
\end{aligned}
$$

The energy equation is

$$
\frac{1}{2} m\left\{\dot{r}^{2} r^{2} \dot{\theta}^{2}\right\}=E_{0}+V_{0}\left(\frac{r_{2}}{r_{1}}-1-\frac{r_{2}}{r}+1\right)
$$

Noting that $\frac{\mathrm{dr}}{\mathrm{d} \theta}=\frac{\dot{\mathrm{r}}}{\dot{\theta}}$ we can rearrange Eq. $(\mathrm{C}-4)$ and combine it with Eq. (C-3) to obtain

$$
\dot{\mathrm{r}}^{2}=\frac{2}{\mathrm{~m}}\left[\mathrm{E}_{0}+\mathrm{V}_{\mathrm{o}}\left(\frac{\mathrm{r}_{2}}{\mathrm{r}_{1}}-\frac{\mathrm{r}_{2}}{\mathrm{r}}\right)\right]-\frac{\mathrm{L}^{2}}{\mathrm{~m}^{2} \mathrm{r}^{2}} .
$$

Integrating Eq. (C-5) gives 


$$
\theta(r)=\sin ^{-1}\left\{-\frac{\frac{V_{1} r_{1} r_{2}}{r_{2}-r_{1}} r-\frac{L^{2}}{m}}{r \sqrt{\left.\left(\frac{V_{1} r_{1} r_{2}}{r_{2}-r_{1}}\right)^{2}+\frac{2}{m} \mid E_{0}+\frac{V_{1} r_{2}}{r_{2}-r_{1}}\right) L^{2}}}\right\} \text {, }
$$

which gives a measure of the angular migration of the electron. We can simplify this result and achieve more quantitative insight if we take the pessimistic extreme of $\mathrm{v}_{\mathrm{r}}=0$. We can then eliminate the explicit dependence on initial angular momentum, and write Eq. (C-6) as

$$
\theta(r) \cong \sin ^{-1}\left\{-\frac{\frac{V_{1} r_{2}}{r_{2}-r_{1}} r+E_{0} r_{1}}{r \sqrt{\left(\frac{V_{1} r_{2}}{r_{2}-r_{1}}\right)^{2}+4\left(E_{0}+\frac{V_{1} r_{2}}{r_{2}-r_{1}}\right) E_{0}}}\right\} \text {. }
$$

Now a measure of the angular blurring is $\Delta \theta=\theta\left(r_{2}\right)-\theta\left(r_{1}\right)$. Using the approximation, $\sin ^{-1}\{x-1\} \cong-\frac{\pi}{2}+\sqrt{x}$, which is good for $x \ll 1$, Eq. (C-7) gives

$$
\Delta \theta \cong \sin ^{-1}\left\{-\frac{\frac{\rho}{p-1}+\frac{2 \varepsilon}{\rho}}{\frac{\rho}{\rho-1}+2 \varepsilon}\right\}+\frac{\pi}{2},
$$

where $\rho=\left(r_{2} / x_{1}\right)>1$ and $\varepsilon=\left(E_{0} / V_{1}\right)<1$. The approximation of Eq. (C-8) may be further simplified to

$$
\Delta \theta \cong 2 \frac{\rho-1}{\rho} \sqrt{\varepsilon},
$$

which is an excellent approximation for $\varepsilon<10^{-3}$ and $\rho>1.005$ which is our region of interest. 
Clearly the smallest angular blurring occurs as $\rho \rightarrow 1$, which is the case of parallel planes and there is no magnification.

\section{APPENDIX D}

EXPLOSION OF LASER IRRADIATED PROTEIN GLOBULES IMBEDDED

IN A TRANSPARENT CYTOPLASM

\section{DEPOSITION OF LASER ENERGY}

The energy deposited in an incremental element of protein immersed in transparent material and exposed to a constant intensity laser beam; $I\left(\mathrm{erg} \cdot \mathrm{cm}^{-2} \cdot \mathrm{s}^{-1}\right)$ can be approximated by

$$
E \cong\left[1-\exp \left(-\Sigma_{a b s} \rho K \Delta x\right)\right] I A t,
$$

where $\Sigma_{\text {abs }}\left(\mathrm{cm}^{2}\right)$ is the absorption cross section for the entire protein globule; $\rho\left(\mathrm{g}^{\circ} \mathrm{cm}^{-3}\right)$ is the density of the protein; $\mathrm{K}\left(\mathrm{gm}^{-1}\right)$ is the number of protein globules per gram; $A\left(\mathrm{~cm}^{2}\right)$ is the area and $\Delta x(\mathrm{~cm})$ is the depth of the incremental element of protein. Formally, this applies to a cylindrical element exposed to a constant intensity laser beam. Now, if the optical depth, $\left(\Sigma_{a b s} \rho K\right)^{-1}$, of the protein globule is large compared to its radius, and every absorbing species within the globule sees the same laser intensity. Eq. (D-1) is well approximated by

$$
E \cong K \Sigma_{a b s} \rho \Delta x A I t
$$

Equation (6) can be further simplified to 
where $m_{p}$ is the mass of the protein globule. The energy given to the globule depends only on the number of absorbing species and is independent of density.

II. EQUATIONS FOR AN EXPLODING PROTEIN GLOBULE

Consider the expansion of a small spherical globule of material embedded within a transparent material of nearly the same density. For easy reference let us call the globule of heated material protein and the surrounding unheated material cytoplasm. The origin of the heating is a laser beam of constant intensity, therefore the energy deposited within the protein globule increases linearly with time until the laser pulse terminates. The rapidly heated protein globule will explode into the cooler surrounding cytoplasm creating a spherically expanding shockwave. 53 It is a strong shockwave because the sound speed in the cytoplasm is low, and the density behind the shock front will be given by

$$
\rho_{\mathrm{s}}=\frac{\gamma+1}{\gamma-1} \rho_{0}
$$

where $\rho_{0}$ is the normal density of the cytoplasm and $\gamma$ is the usual ratio of specific heats $\left(\gamma=C_{p} / C_{v}\right)$. We call the region of higher density cytoplasm the "snowplow" region, because the cytoplasm is swept up as if the exploding protein were a snowplow. He can estimate the thickness, $\Delta r$, of the snowplow region by

$$
4 \pi \mathrm{R}^{2} \Delta \mathrm{r} \rho_{\mathrm{s}}=\frac{4}{3} \pi\left(\mathrm{R}^{3}-\mathrm{R}_{0}^{3}\right) \rho_{\mathrm{o}}, \text { or } \quad \Delta \mathrm{r}=\frac{\left(\mathrm{R}^{3}-\mathrm{R}_{0}^{3}\right)}{3 \mathrm{R}^{2}} \frac{\gamma-1}{\gamma+1} \text {, }
$$

where $R$ is the radius of the shockwave, and $R_{0}$ is the initial radius of the protein globule. When $R \gg R_{0}$, the thickness of the snowplow region is proportional to the radius of the expanding sphere, giving the distribution a selfsimilar character. 
The mass contained in the snowplow region is

$$
M=\frac{4}{3} \pi\left(R^{3}-R_{o}^{3}\right) \rho_{o}
$$

The equation of notion is

$$
\frac{d}{d t}(M U)=4 \pi R^{2} P_{p}
$$

where $P_{p}$ is the pressure inside the expanding sphere and $U$ is the fluid velocity of the snowplow.

Both the protein and cytoplasm are initially cold. To the extent that they can be approximated as polytropic gases with a constant $\gamma$, the initial sound speed must be negligible. Therefore we can approximate the shockwave generated by the exploding protein as a strong shockwave, and we can use the following relations :

$$
U=\frac{2}{\gamma+1} \frac{d R}{d t}
$$

and

$$
P_{s}=\frac{2}{\gamma+1} \rho_{0}\left(\frac{d R}{d t}\right)^{2},
$$

where $P_{s}$ is the pressure behind the shockwave front. We relate the pressure of the shockwave to the pressure inside the expanding sphere by a constant, $\alpha$, which will be determined by requirements of the time-dependence of total energy:

$$
P_{p}=\alpha P_{s}
$$


Substituting Eqs. (D-5) and (D-7) in the left half of Eq. (D-6) and Eqs. (D-8) and $(D-9)$ in the right half, we obtain

$$
\frac{d}{d t}\left[\frac{4}{3} \pi\left(R^{3}-R_{0}^{3}\right) \rho_{0} \frac{2}{\gamma+1} \frac{d R}{d t}\right]=4 \pi R^{2} \alpha \frac{2}{\gamma+1} \rho_{0}\left(\frac{d R}{d t}\right)^{2}
$$

or

$$
\frac{1}{3} \frac{d}{d t}\left[\left(R^{3}-R_{0}^{3} j \frac{d R}{d t}\right]=\alpha R^{2}\left(\frac{d R}{d t}\right)^{2}\right.
$$

Integrating $\mathrm{Eq} \cdot(\mathrm{D}-10)$ gives

$$
\frac{d R}{d t}=a\left(R^{3}-R_{0}^{3}\right)^{\alpha-1}
$$

where a is a constant of integration. To evaluate the two constants a and $\alpha$, we use the approximations that: (1) all of the energy in the snowplow region is kinetic energy and (2) all of the energy in the region inside the expanding sphere is internal energy. The total energy is therefore;

$$
E=\frac{1}{2} M U^{2}+\frac{4}{3} \pi R^{3}\left(\frac{P p}{\gamma-1}\right)
$$

Equation (D-12) turns out to be a good approximation for the degree of accuracy and insight we are trying to achieve. Clearly the two regions contain both internal and kinetic energy. The most serious deficiency is to treat the protein and cytoplasm as a polytropic gas when at lower temperatures it certainly has a more complicated equation of state. Substituting Eqs. (D-5) and (D-7) in the kinetic energy term and Eqs. (D-8) and (D-9) in the internal energy term, we find 


$$
E=\frac{1}{2} \frac{4}{3} \pi\left(R^{3}-R_{0}^{3}\right) \rho_{0}\left(\frac{2}{\gamma+1}\right)^{2}\left(\frac{d R}{d t}\right)^{2}+\frac{4}{3} \pi R^{3} \frac{2 \alpha \rho_{o}}{(\gamma+1)(\gamma-1)}\left(\frac{d R}{d t}\right)^{2},
$$

and using Eq. (D-11) for the derivatives

$$
E=a^{2} \frac{8}{3} \pi \rho_{0}\left[\frac{\left(R^{3}-R_{0}^{3}\right)^{2 \alpha-1}}{(\gamma+1)^{2}}+\alpha R^{3} \frac{\left(R^{3}-R_{0}^{3}\right)^{2 \alpha-2}}{\left(\gamma^{2}-1\right)}\right]
$$

By selecting $\alpha$ such that Eq. (D-14) gives the correct dependence of total energy on time we can evaluate $a$. The constants a and $\alpha$ can then be used in the equation of motion, $E q$. (D-11), to give $R$ as a function of time. We want $E$ to increase linearly with time, but because of different exponents in the kinetic energy and potential energy terms of Eq. (D-14), a general solution is not easily obtained. We therefore look at the early time solution, $\left(R \cong R_{0}\right)$, and the late time solution, $\left(R \gg R_{0}\right)$, for which good approximations can be obtained. Fortunately these are the most interesting approximations for the holography problem.

\section{EARLY-TIME SOLUTION}

First we consider solutions of Eq. (D-11) and Eq. (D-14) when $R \cong R_{0}$. Defining the variable $x \equiv\left(R-R_{0}\right)$, we have

$$
\frac{4}{3} \pi\left(R^{3}-R_{0}^{3}\right) \cong 4 \pi R_{o}^{2} x
$$

or

$$
\left(R^{3}-R_{0}^{3}\right) \cong 3 R_{0}^{2} x
$$

Equation (D-14) can be rewritten 


$$
E=a^{2} \frac{8}{3} \pi \rho_{0}\left[\frac{\left(3 R_{o}^{2} x\right)^{2 \alpha-1}}{(\gamma+1)^{2}}+\alpha R^{2} \frac{\left(3 R_{o}^{2} x\right)^{2 \alpha-2}}{\left(\gamma^{2}-1\right)}\right] .
$$

The internal energy term of Eq. (D-16) will dominate at early time when there has been little motion, so we can ignore the kinetic energy term.

We can write the equation of motion $\left[\mathrm{Eq}_{\mathrm{q}}(\mathrm{D}-11)\right]$ in terms of $\mathrm{x}$ :

$$
\frac{d x}{d t}=a\left(3 R_{0}^{2} x\right)^{\alpha-1} .
$$

Integrating Eq. (D-17), we find

$$
x=\left[\begin{array}{ll}
3 & R_{0}^{2}
\end{array}\right]^{\frac{\alpha-1}{2-\alpha}} \quad[a t(2-\alpha)]^{\frac{1}{2-\alpha}}
$$

Substituting Eq. (D-18) into the second term of Eq. (D-16) we obtain the total energy,

$$
E=a^{2} \frac{8}{3} \pi \rho_{0}\left[\frac{\alpha R_{0}^{3}}{\gamma-1}\right]\left[3 R_{o}^{2}\right]^{2 \alpha-2}\left[\frac{a(2-\alpha) t}{\left(3 R_{0}^{2}\right)^{1-\alpha}}\right]^{\frac{2 \alpha-2}{2-\alpha}}
$$

Now $E$ must increase linearly with time,

so $\frac{2 \alpha-2}{2-\alpha}=1$, or $\alpha=\frac{4}{3}$,

and Eq. ( $\mathrm{b}-18)$ becomes 


$$
x=\sqrt{\frac{8 a^{3}}{9}} R_{o} t^{3 / 2},
$$

and $\mathrm{Eq} \cdot(\mathrm{D}-19)$ becomes

$$
E=a^{3} \frac{64 \pi}{9} \frac{\rho_{o} R_{o}^{5}}{\gamma-1} t .
$$

This is the proper linear dependence of E. Using Eq. (D-3) in Eq. (D-2i) and noting that $\mathrm{m}=\frac{4}{3} \pi \mathrm{R}_{0}^{3} \rho_{0}$, we obtain

$$
a^{3}=\frac{3 K \Sigma_{a b s} I}{16} \frac{(\gamma-1)}{R_{0}^{2}}
$$

Finally, using Eq. (D-22) in Eq. (D-20), we obtain

$$
x=\left[\frac{1}{6}(y-1) K \Sigma_{a b s} I\right]^{1 / 2} t^{3 / 2} .
$$

The radius of the shockwave, $R$, is therefore given by

$$
\begin{aligned}
R & =R_{0}+x \\
& =R_{0}+\left[\hat{f}(\gamma) K \Sigma_{a b s} I\right]^{1 / 2} t^{3 / 2}
\end{aligned}
$$

where

$$
f(\gamma)=\frac{(y-1)}{6} .
$$


We are most interested in the radius of the protein globule, ${ }_{\mathrm{p}}$. Using Eq. (D-7) we can write this as

$$
\begin{aligned}
R_{p} & =R_{0}+\frac{2}{\gamma+1} x \\
& =R_{0}+\left[f(\gamma) K \Sigma_{a b s} I\right]^{1 / 2} t^{3 / 2}
\end{aligned}
$$

where

$$
f(\gamma)=\frac{2}{3} \frac{(\gamma-1)}{(\gamma+1)^{2}}
$$

IV. LATE-TIME SOLUTION

We now consider the solutions of Eq. (D-11) and Eq. (D-14) when $R \gg R_{0}$. Because $R_{0}$ can be neglected when compared with $R$, Eq. (D-14) becomes

$$
E=\frac{8}{3} \pi \rho_{0}\left[\frac{1}{(\gamma+1)^{2}}+\frac{\alpha}{\left(\gamma^{2}-1\right)}\right] R^{3}\left(\frac{d R}{d t}\right)^{2}
$$

and Eq. (D-11) becomes

$$
\frac{d R}{d t}=a R^{3(\alpha-1)},
$$

which when substituted in Eq. (D-26) gives

$$
E=a^{2} \frac{8}{3} \pi \rho_{0} \frac{\alpha(\gamma+1)^{2}+\left(\gamma^{2}-1\right)}{(\gamma+1)^{2}\left(\gamma^{2}-1\right)} \mathrm{R}^{3+6(\alpha-1)} .
$$


Equation (D-28) gives the energy that has been pumped into the protein cytoplasm system through laser-beam energy absorbed in the protein. The energy must increase linearly with time and this occurs if we take $\alpha=7 / 9$. Equation (D-28) becomes

$$
E=\frac{a^{2}}{b} \rho_{0} R^{5 / 3} \quad \text { where } \quad b=\frac{27}{16 \pi} \frac{(\gamma+1)\left(\gamma^{2}-1\right)}{(8 \gamma-1)}
$$

From Eq. (D-27) the assumption that $\alpha=7 / 9$ means

$$
\frac{d R}{d t}=a R^{-2 / 3}
$$

Integrating we find

$$
R=\frac{5}{3} a^{3 / 5} t^{3 / 5}
$$

Substituting Eq. (D-30) into Eq. (D-29) gives

$$
E=\frac{5}{3} \frac{a^{3}}{b} \rho_{0} t
$$

which is the proper dependence of the total energy on time. Substituting Eq.

(D-3) into Eq. (D-31) gives

$$
K \Sigma_{a b s} m_{p} I=\frac{5}{3} \frac{a^{3}}{b} \rho_{0}
$$




$$
a=\left[\frac{4}{5} \pi b K \Sigma_{a b s} I\right]^{1 / 3} R_{0}
$$

Substituting Eq. (D-32) into Eq. (D-30) we obtain the radius of the shockwave

$$
R=\left[\hat{g}(\gamma) K \Sigma_{a b s} I\right]^{1 / 5}\left(R_{0} t\right)^{3 / 5}
$$

where

$$
\hat{\mathbf{g}}(\gamma)=\frac{25}{4} \frac{(\gamma+1)\left(\gamma^{2}-1\right)}{(8 \gamma-1)}
$$

The radius of the protein globule, $R_{p}$, can be estimated by observing that in the strong shockwave limit,

$$
\frac{4}{3} \pi\left(R^{3}-R_{0}^{3}\right) \rho_{0} \cong \frac{4}{3} \pi\left(R^{3}-R_{p}^{3}\right) \rho_{0} \frac{\gamma+1}{\gamma-1} \text {. }
$$

So

$$
R_{p}=\left[g(\gamma) K \Sigma_{a b s} I\right]^{1 / 5}\left(R_{o} t\right)^{3 / 5}
$$

where

$$
g(\gamma)=\left(\frac{2}{\gamma+1}\right)^{5 / 3}\left[\frac{25}{4} \frac{(\gamma+1)\left(\gamma^{2}-1\right)}{(8 \gamma-1)}\right]
$$

V. COMPARISON OF ANALYTIC FORMULAE WITH NUMERICAL CALCULATIONS

At early time the radius of the shockwave and the protein globule are given by Eqs. (D-24) and (D-25) respectively. At late time they are given by Eqs. $(D-33)$ and $(D-34)$. To determine the range of validity of the early-time and 
late-time solutions as well as the overall accuracy of these solutions as a function of $\gamma$, we compare them to numerical simulations using a standard Lagrangian technique. ${ }^{4}$ To accomplish the comparison we take

$$
R_{0}=K \Sigma_{a b s} I=1
$$

Figures $D-1(a), D-1(b)$, and $D-1(c)$ compare the shockwave radius from the numerical calculation with the values given by Eqs. (D-24) and (D-33) for $\gamma=5 / 3$, $4 / 3$, and 1.1 .

The value $\gamma=5 / 3$ corresponds to a monatomic gas and is the largest value likely to be encountered for protein at elevated temperatures and reduced pressure. The value $\gamma=4 / 3$ corresponds to an idealized rigid diatomic molecule, and is representative of an intermediate value for the heated protein or cytoplasm. (In fact, a real diatomic molecule is closer to $\gamma=7 / 5$.) From numerical evaluations of the equation of state of a model protein, $\gamma \cong 1.1$ is a reasonable value for temperatures less than $1000^{\circ} \mathrm{K}$ and densities greater than $10^{-2} \mathrm{~g} \cdot \mathrm{cm}^{-3}$. It therefore represents close to the lowest $\gamma$ expected in the very beginning of the explosion.
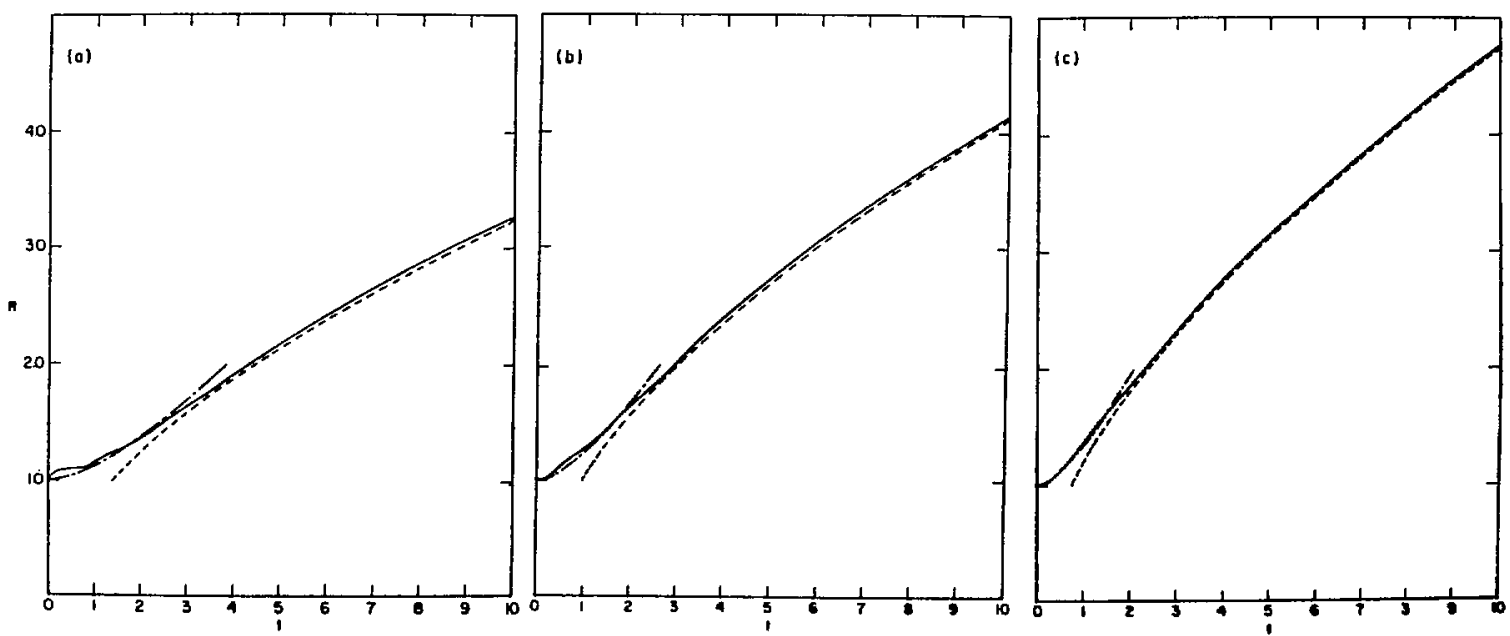

Fig. D-1.

Comparison of shockwave radius calculated by a standard Lagrangian technique with analytic formulae for the early-time expansion [Eq. (C-24)] and the latetime expansion [Eq. (C-33)]. Solid lines are the numerical calculations, dashed lines are the late-time analytic formula and dash-dot-dash lines are the earlytime analytic formula. Figure $D-1(a)$ is for $\gamma=1.1, F i g . D-1(b)$ is for $\gamma=4 / 3$, and Fig. $D-1(c)$ is for $\gamma=5 / 3$. 
From Figs. D-1(a), D-1(b), and D-I(c) it is clear that the early-time solution given by Eq. (D-24) tracks the numerical calculation rather well up to about $R=7 / 4$ at which radius the late-time solution given by $\mathrm{Eq}$. (D-33) becomes more accurate and will be a good fit as $R \rightarrow \infty$. As seen in Figs. (9b) and (9c), the numerical calculation jumps slightly ahead of the analytic solution and then slows down until the analytic solution catches up. This is because the outer Lagrangian zones of the numerical calculation initially expand outward and then slow as a rarefaction propagates to the center of the sphere at which point they begin to expand normally again. To some extent, the numerical calculation represents "reality" for this idealized problem. The effect of the rarefaction propagating to the center is not included in Eq. (D-24).

Figures $D-2(a), D-2(b)$, and $D-2(c)$ show the protein globule interface for $\gamma=5 / 3,4 / 3$, and 1.1. Again a Lagrangian numerical calculation is compared with the early-time solution, Eq. (D-25) and the late-time solution, Eq. (D-34). Again the early-time solution tracks the numerical calculation rather well out to $R_{p} \cong 7 / 4$ after which the late-time solution is a better match. As $\gamma$ in- $^{-}$ creases, the numerical calculation tends to fall behind the analytic solution at late time. This slight inaccuracy is owing to the strong shock approximation. The fluid between the shock front and the protein-cytoplasm interface does not have the full strong-shock compression of $(\gamma+1) /(\gamma-1)$. The inaccuracy fades as $\gamma$ decreases because the compression behind the shock front increases making the value of $R_{p}$ closer to the value of $R$.

Equation (D-25) gives the radius as a function of time for a laser-heated spherical globule of protein embedded in a cytoplasm. It should be accurate as long as $R_{p}<7 / 4 R_{0}$. A useful way to appraise its accuracy near this limiting radius is to compare the doubling time (the time it takes for the radius of the protein globule to double, $R_{p}=2 R_{o}$ ) as a function of intensity and initial radius. From Eq. (D-25), the doubling time is

$$
\mathrm{T}_{2}=\frac{\mathrm{R}_{\mathrm{o}}^{2 / 3}}{\left[\mathrm{f}(\gamma) \kappa \sigma_{\mathrm{abs}} \mathrm{I}\right]^{1 / 3}}
$$



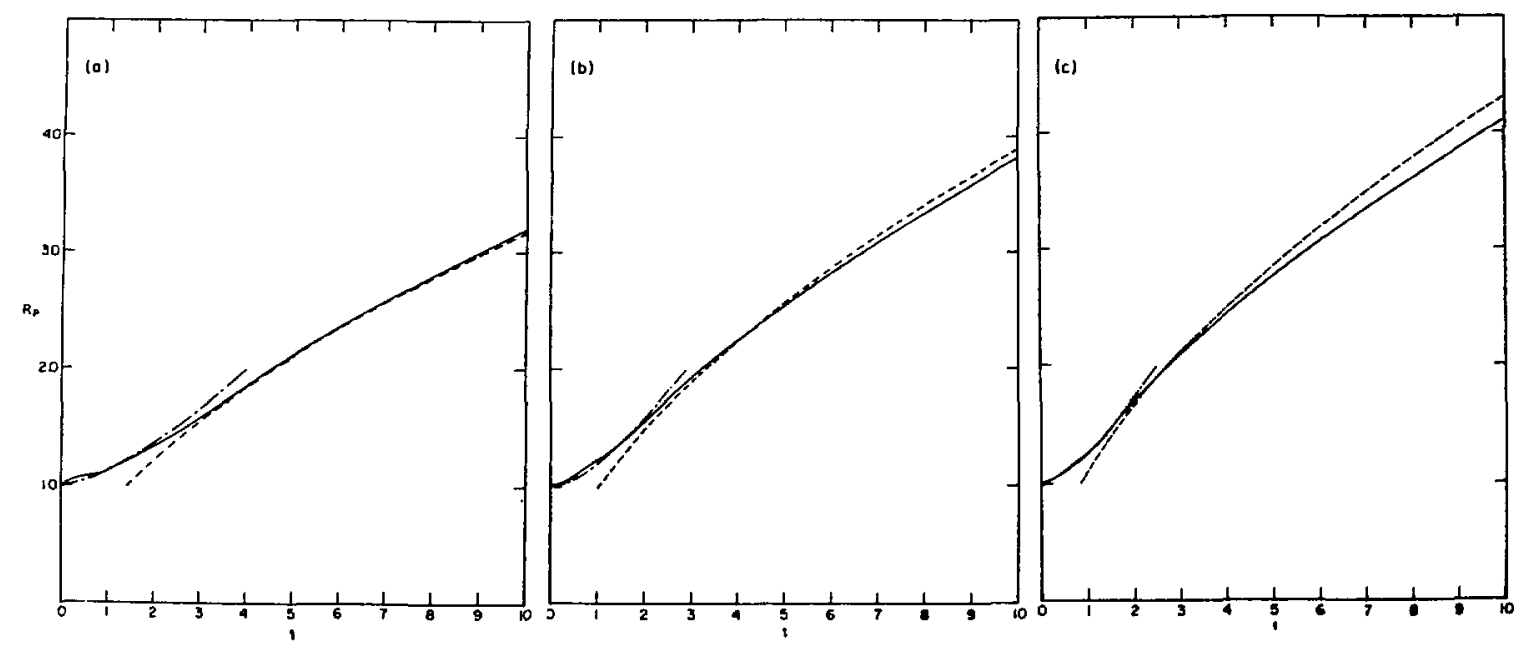

Fig. $D-2$.

Comparison of protein-cytoplasm interface radius calculated by a standard Lagrangian technique with analytic formulae for the early-time expansion [Eq. $(\mathrm{C}-25)]$ and the late-time expansion [Eq. (C-34)]. Solid lines are the numerical calculations, dashed lines are the late-time analytic formula and dash-dot-dash lines are the early-time analytic formula. Figure $D-2(a)$ is for $\gamma=1.1$, Fig. $D-2(b)$ is for $\gamma=4 / 3$, and Fig. $D-2(c)$ is for $\gamma=5 / 4$.

For our standard protein, $K=7.31 \times 10^{21}$ and if we use the nitrogen resonance Discussed in Appendix A, $\sigma_{a b s}=1.7 \times 10^{-17} \mathrm{~cm}^{2}$. Taking $\gamma=5 / 3$, Fig. D-3 compares the analytic doubling time with a numerical calculations as a function of intensity and initial radius.

\section{FREE-RUN EXPANSIONS}

Equations (D-24), (D-25), (D-33), and (D-34) assume a laser turned on at $t=0$ with infinitesimal rise time and remaining on with constant intensity indefinitely. For the case where we may want to preheat a specimen to a state of ionization at which the scattering cross section is increased and the absorption cross section is decreased, we need to know the free-run expansion of the globule after a short laser pulse that injects all the energy at once. If we define the variable $i$ such that

$$
m i=\int_{0}^{\tau} K \Sigma \Sigma_{\text {abs }} I(t) d t
$$




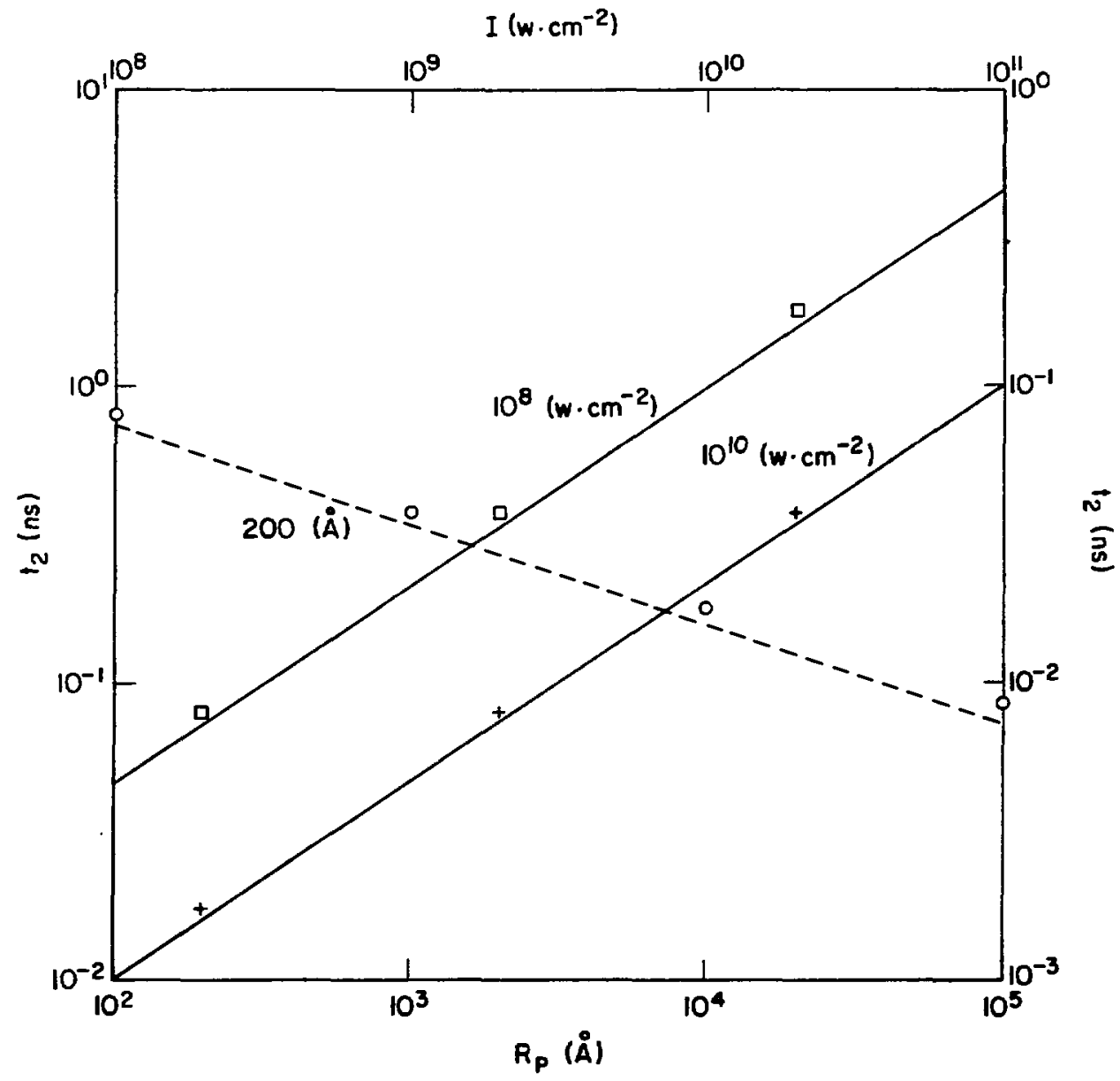

Fig. D-3.

Comparison of the hydrodynamic doubling time (the time required for a laser heated sphere to double in radius) from the analytic formula with one-dimensional numerical calculations using a standard Lagrangian technique. Dashed line from upper left to middle right is the analytic formula at constant radius of $100 \AA$ as a function of intensity, and circles are points from the numerical calculations. Dashed lines from upper right to lower left are the analytic formula at constant intensities of $10^{8}$ and $10^{10} \mathrm{~W} \cdot \mathrm{cm}^{-2}$ as a function of radius, and crosses are points from the numerical calculations.

where $I(t)=0$ for $t>\tau$, and there is negligible change in $R$ or $R_{p}$ during the interval $0<t<\tau$, then we can obtain the early-time and late-time solutions by constraining the total energy to be constant and equal to mi.

For the early-time solution, we constrain Eq. (D-19) to give constant energy equal to $i$. This means we must have $\alpha=1$. Equation $(D-18)$ becomes 
$\mathrm{x}=\mathrm{a} \mathrm{t}$

and Eq. (D-19) becomes

$m i=a^{2} \frac{8}{3} \pi \rho_{o} \frac{R_{o}^{3}}{\gamma-1}$.

The early-time shockwave radius is therefore

$$
R=R_{0}+\sqrt{3 \hat{f(\gamma) i}} t,
$$

and the radius of the protein globule is

$$
R_{p}=R_{0}+\sqrt{3 f(\gamma) i} t .
$$

For the late-time solution we constrain the energy to be constant in $\mathrm{Eq}$. (D-14), which occurs if $\alpha=\frac{1}{2}$. Then Eq. (D-30) becomes

$$
R=\left(\begin{array}{ll}
5 & a
\end{array}\right)^{2 / 5} t^{2 / 5},
$$

and $\mathrm{Eq} \cdot(\mathrm{D}-32)$ becunes

$$
a=\left[\frac{(\gamma+1)\left(\gamma^{2}-1\right)}{(3 \gamma-1)} R_{0}^{3} i\right]^{1 / 2}
$$

The late-time shockwave radius is therefore 


$$
R=\left[\frac{8 \gamma-1}{3 \gamma-1} \hat{g}(\gamma) R_{0}^{3} i\right]^{1 / 5} t^{2 / 5}
$$

and the radius of the protein globule is

$$
R_{p}=\left[\frac{8 \gamma-1}{3 \gamma-1} g(\gamma) R_{0}^{3} i\right]^{1 / 5} t^{2 / 5} .
$$

Equations (D-36) and (D-37) show the linear dependence on time we would expect from dimensional analysis and Eqs. (D-38) and (D-39) show the $t^{5 / 2}$ that could also get from dimensional analysis. Equation (D-38) is the famous "fireball formula", cherished by those who build and test atom bombs.

APPENDIX E

THE EFFECT OF COHERENCE AND SPECIMEN VOLUME ON RESOLUTION

\section{ON-AXIS FRESNEL TRANSFORM}

\section{A. Temporal Coherence Length}

Figure E-1 illustrates a simple way to calculate the effect of temporal coherence. As shown in Fig. E-1(a), the temporal coherence of the laser beam (as measured by a wavepacket length, $x$ ) must be sufficient for the diffracted or scattered wavepacket to at least partially interfere with the unscattered wavepacket. The part of scattered wavepacket that overlaps with the original wavepacket produces the signal. That part that does not overlap produces noise. We are seeking the signal-to-noise ratio as a function of the wavelength $(\lambda)$, the linear resolution $(\delta)$, the distance from the specimen to the recording surface (b), and the wavepacket length ( $x$ ).

Figure E-1(b) shows how the reference and scattered wave packets overlap. When the unscattered wave packet reaches the screen, the scattered wave packet 
(a)

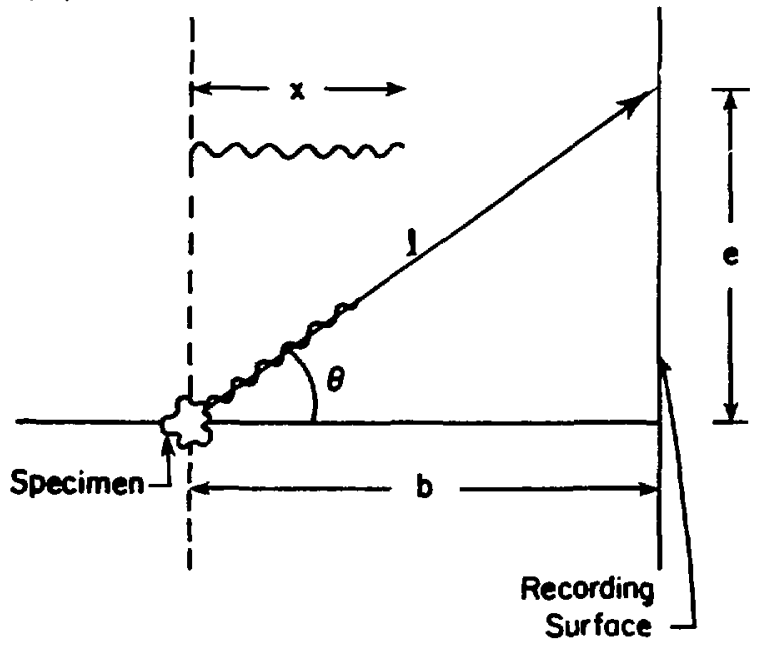

Fig. E-1.

Pathlength difference that results in degradation of resolution with reduction in coherence (wavepacket) length. Figure E-1(a) shows actual geometry with: $x=$ coherence length; $b=$ distance from specimen to recording surface; $\ell=$ distance traveled by diffracted or coherently scattered wavepacket. Figure E-1(b) shows overlap (d) of diffracted and reference wavepacket. Overlapping sections produce

(b) an interpretable signal while nonoverlapping sections produce noise.

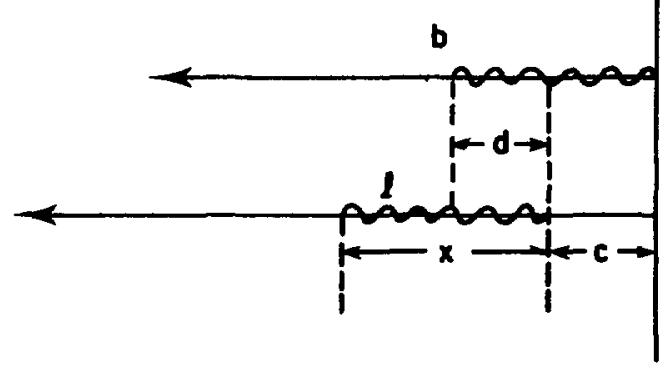

is still a distance $c=(\ell-b)$ away from the screen. The overlap length is $d=(x-c)=(x-l+b)$. The overlap fraction is $\max \{d / x, 0\}=\max \{1+(b-l) / x, 0\}$. Now $b=\ell \cos \theta$, but $\sin \theta=(\lambda / \delta)$ because $\delta$ can be regarded as the spacing of a linear grating. So, $\cos \theta=\left[1-(\lambda / \delta)^{2}\right]^{\frac{3}{2}}$ and the overlap fraction, which is the signal, is given by

$$
S=\max \left\{0,1+\frac{b}{x}\left(1-\left[1-(\lambda / \delta)^{2}\right]^{-\frac{1}{2}}\right)\right\} .
$$

The nonoverlapping part of the signal is noise 


$$
N=\min \left\{1, \frac{b}{x}\left(\left[1-(\lambda / \delta)^{2}\right]^{-\frac{1}{2}}-1\right)\right\}
$$

and the signal-to-noise ratio is

$$
\xi=\frac{S}{S+N+n}
$$

where $\mathrm{n}$ is the noise owing to all other sources. The measure of $\mathrm{n}$ is such that $n=1$ when the noise intensity is equal to the laser intensity. For the moment we will assume that $n=0$.

From Eqs. (E-1), E-2), and (E-3), we can obtain a constraint on the maximum allowable distance between specimen and recording surface as a function of signal-to-noise threshold (the value of $\xi$ at which the fringe pattern becomes interpretable).

$$
\mathrm{b} \leq \frac{(1-\xi) \mathrm{x}}{\left[1-(\lambda / \delta)^{2}\right]^{-\frac{1}{2}}-1}
$$

\section{B. Spatial Coherence Length}

The reference beam must be spatially coherent over the diameter of the hologram. Referring to Fig. E-1(a), the distance from the center of the hologram to a sample interference point is given by

$$
e=b \tan \theta=b \frac{\lambda}{\delta}\left[1-(\lambda / \delta)^{2}\right]^{-\frac{1}{2}}
$$

The spatial coherence length (y) must exceed $2 e$, so we require that

$$
y \geq 2 b \frac{\lambda}{\delta}\left[1-(\lambda / \delta)^{2}\right]^{-\frac{\lambda}{2}}
$$


C. Maximum Volume of the Specimen

Coherence length sets a limit on the volume of the specimen we can holograph. We must have the number of areal resolution elements on the recording surface greather than the number of volume resolution elements in the specimen. The usual recording surface is

$$
\pi e^{2}=\pi b^{2} \frac{\lambda^{2}}{\delta^{2}}\left[1-(\lambda / \delta)^{2}\right]^{-1}
$$

If $\Delta^{2}$ is the areal resolution element, then the number of usabie areal resolution elements on the recording surface is

$$
\pi \frac{b^{2} \lambda^{2}}{\Delta^{2} \delta^{2}}\left[1-(\lambda / \delta)^{2}\right]^{-1}
$$

The number of volume resolution elements in the specimen is

$$
\frac{V}{\delta^{3}}
$$

where $V$ is the specimen volume. We therefore must have

$$
V \leq \frac{\pi \delta b^{2} \lambda^{2}}{\Delta^{2}\left[1-(\lambda / \delta)^{2}\right]}
$$

or using Eq. (E-4)

$$
V \leq \frac{\pi \delta \eta^{2}(1-\xi)^{2} x^{2}}{\Delta^{2}\left(\left[1-(\lambda / \delta)^{2}\right]^{\frac{1}{2}}-1\right)^{2}}
$$


Equation (E-5) can be expanded into a quartic in linear resolution ( $\delta$ ) and solved for the maximum resolution obtainable as a function of wavelength $(\lambda)$, coherence length $(x)$, resolution of the recording surface $(\Delta)$, and volume of the specimen $(V)$. If $\delta \leq \lambda$, the solution of Eq. $(E-5)$ is meaningless because $\delta$ is limited by diffraction. The coherence lergtt, nt:essary for $\delta$ to be limited by diffraction can be found from $\mathrm{Eq} .(\mathrm{E}-5)$ to be

$$
x \geq \frac{\Delta}{1-\xi} \sqrt{\frac{V}{\pi \lambda^{3}}}
$$

If the coherence length satisfies this inequality, then coherence is not a limiting factor in resolution of the hologran.

\section{OFF-AXIS FRESNEL TRANSFORM}

\section{A. Temporal Coherence Length}

Figure E-2 shows a schematic simplified view of how an off-axis Fresnel transform holog:am could be arranged. The laser beam enters from the left and has a total diameter of $2 e+f$. Part of the beam passes through the shadow screen, illuminates the specimen, and is diffracted onto the recording surface. The rest of the beam if reflected by the mirror and it impinges the recording surface at an angle $\theta_{r}$. The radius of a circle on the recording surface illuminated by the reference beam is $e$.

In order for there to be no interference between the virtual and real images in reconstruction, the angle between the reference beam and the recording surface $\left(\theta_{\mathbf{r}}\right)$ must satisfy the inequality

$$
\theta_{r} \geq \sin ^{-1}(\lambda / \delta)
$$

To minimize the effect of coherence length, the mirror must be butted up against the recording surface at the very edge of the area illuminated by the reference beam. A plane parallel to the recording surface ac a distance $2 i j$ in front of 


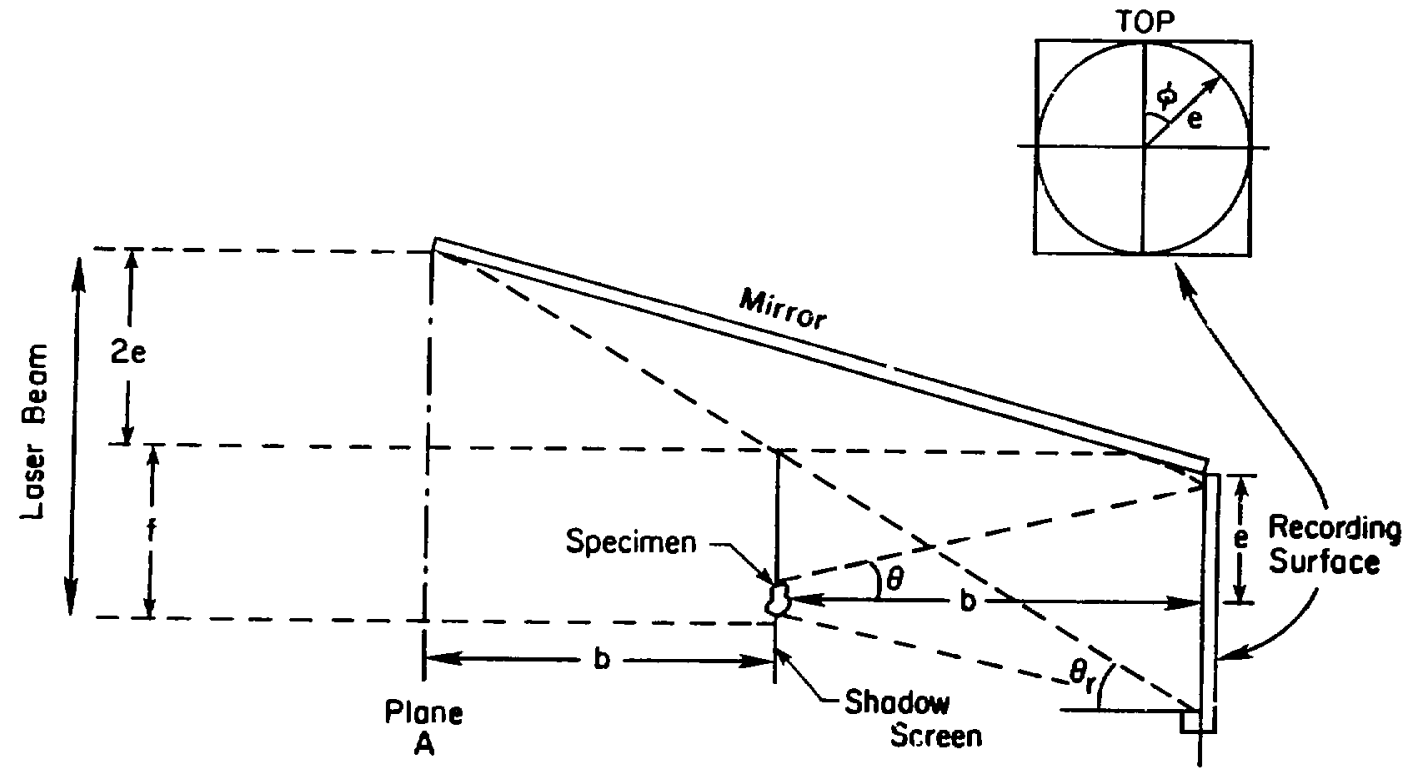

Fig. E-2.

Geometry for calculating coherence length requirements of off-axis Fresnel transform holography.

the recording surface, marks the position where the beam can first impinge the mirror. In Fig. E-2, this is called plane A. The distance the reference beam must travel from plane $A$ to the recording surface is given by

$$
g(\phi)=b \cos \phi\left(1-\sec \theta_{r}\right)+b\left(1+\sec \theta_{r}\right)
$$

where $\phi$ is the azimuthal angle measured from the top of the recording suriace. The distance from $p$ lane $A$ t $u$ the recording surface of the part of the laser beam that is scattered by the specimen into an angle $\theta$ is given by

$$
\ell=b(1+\sec \theta)
$$

The difference in path lengths is given by 


$$
\begin{aligned}
c & =\ell-g(\phi) \\
& =b(1+\sec \theta)-b \cos \phi\left(1-\sec \theta_{r}\right)-b\left(1+\sec \theta_{r}\right) .
\end{aligned}
$$

Now the maximum scattering angle is

$$
\theta=\sin ^{-1}(\lambda / \delta)
$$

Clearly the smallest allowable difference in length would be obtained when $\theta_{r}=\theta$. This is the minimum angle for the reference beam that will ensure separation between the virtual and real images in reconstruction.

Given a wavepacket of temporal coherence length $x$ that is spatially coherent over the plane $A$, the overlap between the scattered and reference part of this wavepacket at the recording surface will be given by $d=(x-c)$, and the signal is given by

$$
\begin{aligned}
S(\phi) & =\frac{d}{x} \\
& =\max \left\{0,1+\frac{b}{x}|\cos \phi|(1-\sec \theta)\right\} \\
& =\operatorname{Lin}\left\{\left\{0,1+\frac{b}{x}|\cos \phi|\left(1-\left[1-(\lambda / \delta)^{2}\right]^{-\frac{1}{2}}\right)\right\} .\right.
\end{aligned}
$$

The nonoverlapping part is the noise.

$$
\mathrm{N}(\phi)=\min \left\{1, \frac{\mathrm{b}}{\mathrm{x}}|\cos \phi|\left(\left[1-(\lambda / \delta)^{2}\right]^{-\frac{1}{2}}-1\right)\right\}
$$

Notice that at the top of the recording surface $(\phi=0)$ and at the botton of the recording surface $(\phi=\pi)$ the equations for signal and noise reduce identically 
to Eqs. (E-1) and (E-2). Also note that at the far right and left sides of the recording surface $(\phi=\pi / 2$ and $\phi=3 / 2 \pi)$ there is perfect overlap of the reference and scattered beams. This is why (all other parameters held the same) a better signal-to-noise will be obtained with the off-axis geometry than with the on-axis geometry. Signal-to-noise is a function of position on the recording surface, but a useful measure of the signal can be obtained by averaging over $\phi$.

$$
\bar{S}=\frac{\int_{0}^{\pi / 2} S(\phi) d \phi}{\int_{0}^{\pi / 2} d \phi} .
$$

Using the fact that $\mathrm{S}(\phi)+\mathrm{N}(\phi)=1$ and assuming no other sources of noise, we obtain the average signal-to-noise threshold:

$$
\xi=\max \left\{0,1+\frac{2 b}{\pi x}\left(1-\left[1-(\lambda / \delta)^{2}\right]^{-\frac{1}{2}}\right)\right\}
$$

Therefore maximum allowable distance between specimen and recording surface is given by

$$
b \leq \frac{\pi(1-\xi) x}{2\left(\left[1-(\lambda / \delta)^{2}\right]^{-\frac{1}{2}}-1\right)}
$$

which is analogous to Eq. $(E-4)$.

\section{B. Spatial Coherence Length}

The off-axis geometry requires a larger spatial coherence length than the on-axis geometry. In the on-axis geometry, the beam diameter required to provide a reference $2 e$ in diameter is also $2 e$. In off-axis geometry a separate part of the beam must be split to illuminate the specimen. The minimum diameter of the laser beam is $3 e$ and the spatial coherence requirement becomes 


$$
\left.\mathrm{y} \geq 3 \mathrm{~b} \frac{\lambda}{\delta}[1-\lambda / \delta)^{2}\right]^{-\frac{1}{2}}
$$

C. Maximum Volume of Specimen

By applying the same argument that give Eq. (E-5), we find the maximum volume in the off-axis geometry to be

$$
V \leq \frac{\pi^{3} \delta \lambda^{2}(1-\xi)^{2} x^{2}}{4 \Delta^{2}\left(\left[1-(\lambda / \delta)^{2}\right]^{\frac{1}{2}}-1\right)^{2}}
$$

and the coherence length necessary to be diffraction limited is

$$
x \geq \frac{2}{\pi} \frac{\Delta}{1-\xi} \quad \frac{V}{\pi \lambda^{3}}
$$

III. PLANAR FOURIER TRANSFORM

\section{A. Temporal Coherence Length}

Referring to Fig. 7 , the best contrast ratio will be obtained if the zone plate diameter is about the same as the specimen diameter. The spatial coherence length requirement is least if the specimen and zone plate are as close together as possible. Therefore, the center-to-center spacing between the specimen and zone plate is about $a$. If $b \gg a$, the problem of calculating the effect of temporal coherence is greatly simplified; the zone plate focal point can be considered to be at the same position as the specimen, and the difference in path length of the scattered and reference waves at the perimeter of the tologram is given by

$$
\sqrt{e^{2}+b^{2}}-\sqrt{e^{2}+b^{2}+a^{2}-2 a e \cos \phi}
$$


The signal is a function $\phi$ as for the off-axis Fresnel transform case.

$$
\mathrm{S}(\phi) \cong \max \left\{0,1-\frac{\mathrm{a}^{2}\left[1-(\lambda / \delta)^{2}\right]}{2 \mathrm{bx}}+\frac{\mathrm{a} \lambda}{\mathrm{x} \delta} \cos \phi\right\}
$$

The average signal obtained from Eq. $(\mathrm{E}-7)$ is

$$
\bar{S}=1-\frac{\left.a^{2}[1-\lambda / \delta]^{2}\right]^{\frac{1}{2}}}{2 b x}
$$

So the temporal coherence requirement is

$$
x \geq \frac{a^{2}}{2 b(1-\xi)}\left[1-(\lambda / \delta)^{2}\right]^{\frac{1}{2}}
$$

Notice that for the Fresnel transform case, $x$ is proportional to $b$ and in the Fourier transform case $x$ is inversely proportional to $b$.

\section{B. Spatial Coherence Length}

The $x$-ray beam must be spatially coherent over the specimen and the zone plate. The requirement is thus

$$
\mathrm{y} \geq 2 \mathrm{a}
$$

Unlike Fresnel transform holography, the spatial coherence can be very small for microscopic specimens.

\section{Maximum Volume of Specimen}

Because $x$ is inversely proportional to $b$, the specimen volume constraint cannot be written in a single expression. The specimen volume is $\frac{1}{6} \pi a^{3}$, so from Eq. (11) 


$$
V \leq \frac{\pi}{6}\left[1-(\lambda / \delta)^{2}\right]^{-3 / 4}[2 \operatorname{xb}(1-\xi)]^{3 / 2}
$$

We call this the "coherence limit".

Another constraint can be applied from the requirement that there be an areal resolution element on the recording surface for each volume resolution element in the specimen; i.e., $V / \delta^{3} \leq \pi e^{2} / \Delta^{2}$. From Eq. (E-8) this becomes

$$
V \leq \frac{\pi b^{2} \lambda^{2} \delta}{\Delta^{2}\left[1-(\lambda / \delta)^{2}\right]}
$$

which we call the "mapping limit". The maximum volume is the minimum value given by Eqs. $(E-9)$ and $(E-10)$. The volume can be made indefinitely large by increasing $b$.

IV. SPHERICAL FOURIER TRANSFORM

\section{A. Temporal Coherence Length}

Referring to Fig. 8 and again assuming that $b \gg$, the difference in path length of the scattered and reference waves at the perimener of the hologram is given by

$$
b-\sqrt{b^{2}+a^{2}-2 a b(\lambda / \delta) \cos \phi},
$$

and the signal is

$$
\mathrm{S}(\phi) \cong \max \left\{0,1-\frac{\mathrm{a}^{2}}{2 \mathrm{xb}}+\frac{\mathrm{a} \lambda}{2 \mathrm{x} \delta} \cos \phi\right\}
$$

and the average signal is 


$$
\overline{\mathrm{S}}=1-\frac{\mathrm{a}^{2}}{2 \mathrm{xb}}
$$

so the temporal coherence requirement is

$$
x \geq \frac{a^{2}}{2 b(1-\xi)}
$$

\section{B. Spatial Coherence Length}

Again, the spatial coherence must span the reflector and the specimen, even when they are right next to each other, so

$$
y \geq 2 a
$$

C. Maximum Volume of Specimen

The temporal coherence length requirement, Eq. $(\mathrm{E}-11)$ implies

$$
V \leq \frac{\pi}{6}[2 \operatorname{xb}(1-\xi)]^{3 / 2}
$$

which is the "coherence limit".

The "mapping limit" derives from $V / \delta^{3} \leq 2 \pi \mathrm{bh} / \Delta^{2}$, where $h$ is the rise of the spherical shell. This gives

$$
V \leq \frac{2 \pi b^{2} \delta^{3}}{\Delta^{2}}\left\{1-\left[1-(\lambda / \delta)^{2}\right]^{\frac{1}{2}}\right\}
$$

Again, the maximum volume is given by the lesser of the values of Eqs. (E-12) and $(E-13)$. 
EFFECT OF DOPPLER BROADENING ON RESOLUTION IN FRESNEL TRANSFORM HOLOGRAPHY

I. MAXIMUM DISTANCE FROM SPECIMEN TO RECORDING SURFACE

If the specimen is sufficiently transparent for the hologram to be generated by scattering from individual atoms, then the Doppler shift that results from thermal motion of those atoms can shift the phase of scattered radiation at the recording surface and degrade the resolution. Referring to $F i g$. (F-1), we see that

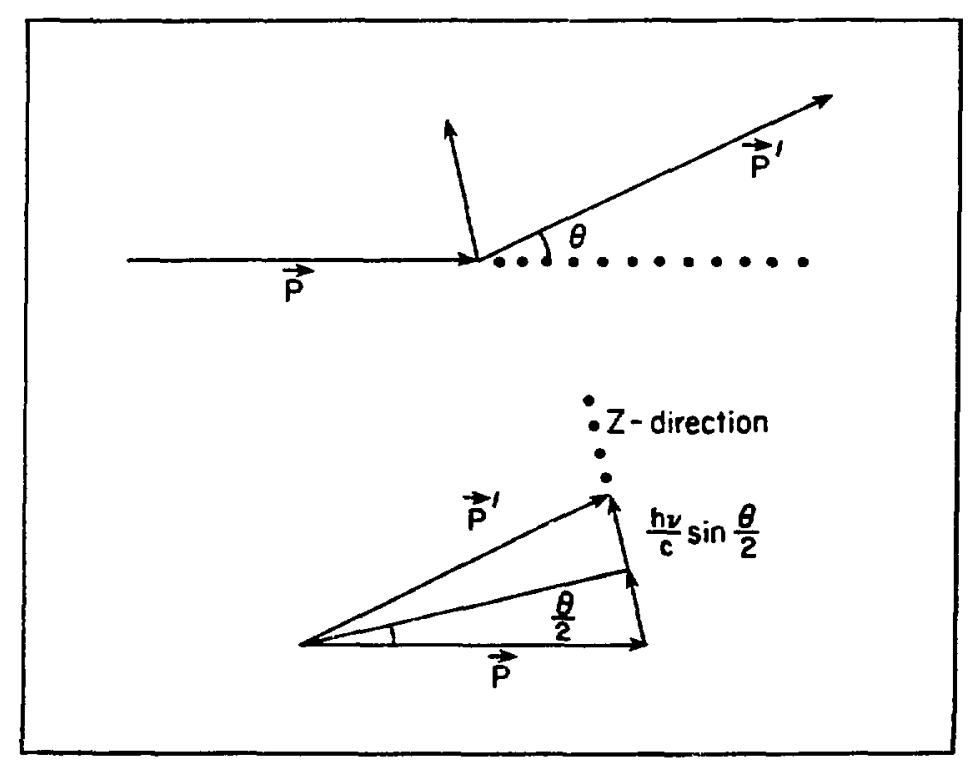

Fig. F-1.

Vector diagram of scattering of a photon from a moving atom, which results in a slight Doppler shift of the photon energy. 


$$
\begin{aligned}
\Delta E & =\frac{|\vec{P}|^{2}-|\vec{P} \cdot|^{2}}{2 M} \\
& \cong \frac{4 \frac{h v}{c} P_{z} \sin \frac{\theta}{2}}{2 M}
\end{aligned}
$$

where $\vec{P}$ is the momentum and $M$ is the mass of the atom with which the photon, hv, collides. The spread in wavelength that results from numerous scattering is obtained from Eq. $(F-1)$ as

$$
\begin{aligned}
\frac{\Delta \lambda}{\lambda} \cong \frac{\Delta E}{h v} \\
\quad=2 \frac{\bar{v}}{c} \sin \frac{\theta}{2}
\end{aligned}
$$

where $\bar{v}_{z}$ is the average atom velocity in the z-direction as defined in Fig. F-1, and $c$ is the speed of light. We can replace $\vec{v}_{z}$ with $\sqrt{\frac{1}{3}} \bar{v}$ where $\bar{v}$ is the mean atomic velocity. Using the geometry in Fig. E-1, the phase blurring at the recording surface is given by

$$
\begin{aligned}
\Delta \phi & =2 \pi \ell \frac{\Delta \lambda}{\lambda^{2}} \\
& =\frac{4 \pi}{\sqrt{3}} \frac{\ell}{\lambda} \frac{\vec{v}}{\mathrm{c}} \sin \frac{\theta}{2} .
\end{aligned}
$$

A reasonable measure of the signal is

$$
S=e^{-\frac{\Delta \phi}{\pi}}
$$


and the noise from phase blurring is

$$
N=1-S \text {. }
$$

The signal-to-noise ratio is then

$$
\begin{aligned}
\xi & =\frac{S}{S+N+n} \\
& =e^{-\frac{\Delta \phi}{\pi}}
\end{aligned}
$$

assuming there are no other sources of noise $(n=0)$. Combining Eqs. (F-3) and $(\mathrm{k}-4)$

$$
\ln \xi=-\frac{4}{\sqrt{3}} \frac{a}{\lambda} \frac{\bar{v}}{c} \sin \frac{\theta}{2}
$$

Now from Fig. (14a), $b=a \cos \theta$, and $\sin \theta=(\lambda / \delta)$ because $\delta$ can be regarded as the spacing of a linear grating. Also, $\cos \theta=\left[1-(\lambda / \delta)^{2}\right]^{\frac{1}{2}}$, and, using the half-angle formula, we can rewrite Eq. $(\mathrm{F}-5)$ as

$$
\mathrm{b} \leq-\frac{\sqrt{6}}{4} \lambda \frac{\mathrm{c}}{\bar{v}}\left[\frac{1-(\lambda / \delta)^{2}}{1-\left[1-(\lambda / \delta)^{2}\right]^{\frac{3}{2}}}\right]^{\frac{2}{2}} \ln \xi .
$$

If $\lambda \ll \delta, E q .(F-6)$ simplifies to

$$
b \leq-\frac{\sqrt{3}}{2} \frac{c}{\mathrm{v}} \delta \ln \xi
$$


II. MAXIMUM VOLUME OF SPECIMEN

Using the argument given in Appendix E, the volume of the specimen is constrained to

$$
V \leq \frac{\pi \delta b^{2} \lambda^{2}}{\Delta^{2}\left[1-(\lambda / \delta)^{2}\right]}
$$

where $\Delta^{2}$ is the areal resolution element of the recording surface. Using Eq. $(\mathrm{F}-6)$ in $\mathrm{Eq} .(\mathrm{F}-8)$ and writing $\overline{\mathrm{v}}=\mathrm{k}(\mathrm{T} / \mathrm{A})^{\frac{1}{2}}$, where $\mathrm{T}$ is the temperature, $\mathrm{A}$ is the atomic weight of the scattering atom, and $\mathrm{k}$ is a constant depending on the units in which $\mathrm{T}$ is measured $\left(\mathrm{k}=1.45 \times 10^{4}\right.$ if $\mathrm{T}$ is in ${ }^{\circ} \mathrm{K}$, or $\mathrm{k}=1.56 \times 10^{6}$ if $\mathrm{r}$ is in $\mathrm{eV}$ ) we obtain

$$
V \leq \frac{3 \pi \delta \lambda^{4} c^{2} A \ell n^{2} \xi}{8 T k^{2} \Delta^{2}\left\{1-\left[1-(\lambda / \delta)^{2}\right]^{\frac{1}{2}}\right\}}
$$

Equation ( $F-9)$ can be solved numerically for the maximum resolution obtainable $(\delta)$ in terms of the specimen volume $(V)$, the wavelength $(\lambda)$, the atomic weight of the scattering atoms (A), the areal resolution element of the recording surface $\left(\Delta^{2}\right)$, the specimen temperature $(\mathrm{T})$, and the signal-to-noise threshold $(\xi)$. If $\delta \leq \lambda$, the solution of Eq. $(F-9)$ is meaningless because $\delta$ is diffraction limited. The temperature below which $\delta$ is limited by diffraction is found from Eq. $(F-9)$ to be

$$
T \leq \frac{3 \pi \delta \lambda^{4} \mathrm{c}^{2} \mathrm{~A} \ell \mathrm{n}^{2} \xi}{8 \mathrm{k}^{2} V \bar{\Delta}^{2}}
$$

If the temperature satisfies this inequality, then Doppler broadening is not a limiting factor in resolution of the hologram. 
The results given in Eqs. $(F-6),(F-9)$, and $(F-10)$ assume that the bologram is generated by coherent scattering on the atomic level If, however, we are holographing a specimen consisting of an array of nearly opaque objects, most of the scattering is diffraction around these objects. The diffractive scat_ering from a completely opaque object will not. be subject to thermal Doppler broadening, although it may have a Doppler shift if the object is moving or expanding. A crude but reasonable way to estimate the resolution lost to phase blurring in a semiopaque object is to divide the total scattering into two components: (1) shadow coherent scattering, which is not subject to Doppler broadening, the cross section for which increases as the square of the extinction chrough the object times the geometric cross section; and (2) atomic cot rent scattering, which is subject to Doppler broadening, the cross section for which is the sum of the coherent scattering cross sections of the atoms within the object times the transmission. If $\Sigma_{2}$ is the total cross section for shadow coherent scattering and $\Sigma_{1}$ is the total cross section for atomic coherent scattering, then the signal is

$$
S=\frac{\Sigma_{1} e^{-\frac{\Delta \phi}{\pi}}+\Sigma_{2}}{\Sigma_{1}+\Sigma_{2}} .
$$

Again we have $\mathrm{S}+\mathrm{N}=1$, so

$$
\xi=\alpha e^{-\frac{\Delta \phi}{\pi}}+\beta
$$

where

$$
\alpha=\frac{\Sigma_{1}}{\Sigma_{1}+\Sigma_{2}} \quad \text { and } \beta=\frac{\Sigma_{2}}{\Sigma_{1}+\Sigma_{2}} \text {. }
$$


So, for a semiopaque specimen, Eqs. $(F-6),(F-9)$, and $(F-10)$ are still valid if we replace $\ln \xi$ by $\ln \left(\frac{\xi-\beta}{\alpha}\right)$.

IV. DOPPLER BROADENING FOR CTHER HOLOGRAPHIC TECHNIQUES

Because the temporal coherence length of the reference beam is assumed to be long compared to the temporal coherence length of the scattered beam, Eqs. $(F-6)$ and (F-9) are correct for all forms of the holography for which the specimen is backlighted and the hologram is registered on a planar recording surface, Of the four types of holography $I$ have considered, only spherical Fourier transform cannot be described by Eqs. $(F-6)$ and $(F-9)$. This holographic technique, $a=b$ in $\mathrm{Eq} \cdot(\mathrm{F}-5)$ so the inequality analngous to $\mathrm{Eq} \cdot(\mathrm{F}-6)$ is

$$
\mathrm{b} \leq-\frac{\sqrt{6}}{4} \lambda \frac{\mathrm{c}}{\mathrm{v}}\left\{1-\left[1-(\lambda / \delta)^{2}\right]^{\frac{1}{2}}\right\}^{-\frac{1}{2}} \text { ln } \xi
$$

and the inequality analogous to Eq. $(F-9)$ is

$$
\mathrm{V} \leq \frac{3 \pi \delta \lambda^{4} c^{2} A \ln ^{2} \xi}{8 \mathrm{~T} \mathrm{k}^{2} \Delta^{2}\left\{1-(\lambda / \delta)^{2}\right\}\left\{1-\left[1-(\lambda / \delta)^{2}\right]^{\frac{3}{2}}\right\}}
$$

Extension of the treatment for semiopaque specimens is straightforward.

The treatment in this section can also be easily extended to other forms of incoherent scattering as long as the reference beam is much more coherent than the scattered beam and the signal-to-noise ratio is well described by a phase blurring as in Eq. $(F-4)$. 
APPENDIX G

THE EFFECT OF HEAT TRANSPORT ON HYDRODYNAMIC BLURRING

The resuits given in Appendix $D$ are actually a worst case approximation from the standpoint of hydrodynamic blurring. They assume that the only loss of energy from the protein globule is owing to mechanical work done on the cytoplasin. In fact, energy is lost from the protein globule owing to (1) radiation transport, (2) thermal conductivity, and (3) transport of fast electrons.

\section{RADIATION TRANSPORT}

In general, temperatures encountered in the explosion of protein globules will not be high enough to make radiation a significant energy loss mechanism. The radiant energy flux from the surface of a perfect black body is

$$
s=\sigma T^{4}
$$

where $\sigma$ is the Stefan-Boltzman constant. Because of the $\mathrm{T}^{4}$ dependence, not much energy is lost over the time intervals of interest. For example, consider a standard protein molecule at $5 \times 10^{4}{ }^{\circ} \mathrm{K}$. If its density is $1 \mathrm{gm}^{\circ} \mathrm{cm}^{-3}$, a numerical calculation of the equation of state gives an internal energy of $9.02 \times 10^{11}$ erg.gn ${ }^{-1}$. The molecule is $25 \AA$ in radius, so its mass is $6.54 \times 10^{-20} \mathrm{gm}$ and its surface area is $7.85 \times 10^{-13} \mathrm{~cm}^{2}$. The total internal energy in the molecule is $5.90 \times 10^{-8} \mathrm{erg}$ and the energy loss rate to radiation is $2.78 \times 10^{2} \mathrm{erg} \cdot \mathrm{s}^{-1}$. In general, the longest time we would observe such an object is $10^{-11} \mathrm{~s}$, in which time it would lose about $5 \%$ of its energy to radiation.

This simple analysis assumes (1) both protein and cytoplasm are in local thermodynamic equilibrium, (2) the protein is opaque to its own thermal radiation, and (3) the cytoplasm is transparent to the thermal radiation. In fact, opacities are such that the situation is better described by a radiation diffusion front coupled with the shockwave and expansion described in Appendix $C$. However, to the extent that this analysis is reasonable, the fractional rate of energy lost to radiation goes as the cube of the temperature, inversely as the radius of the protein globule, and inversely as its density. 


\section{THERMAL CONDUCTIVITY}

Thermal conductivity is a complicated function of density and temperature, but as the specimen approaches the plasma state, heat lost by conductive mechanisms will be dominated by the electronic conductivity, which varies as the $5 / 2$ power of temperature. This suggests that radiation will dominate at high temperature and thermal conductivity will dominate at low temperature. The situation is complicated, but in general conductive losses will not be important. Figure G-1 shows a numerical calculations for the expansion of a sphere of material containing $\mathrm{H}, \mathrm{C}, \mathrm{O}$, and $\mathrm{N}$ in proportions not drastically dissimilar to protein, imbedded in the same unheated material. The equation of state and thermal conductivity are normalized to experimental data. One curve shows the expansion with thermal conductivity included in the calculation and the other with thermal conductivity shut off. The curves diverge only slightly and only in the late stages of the calculation.

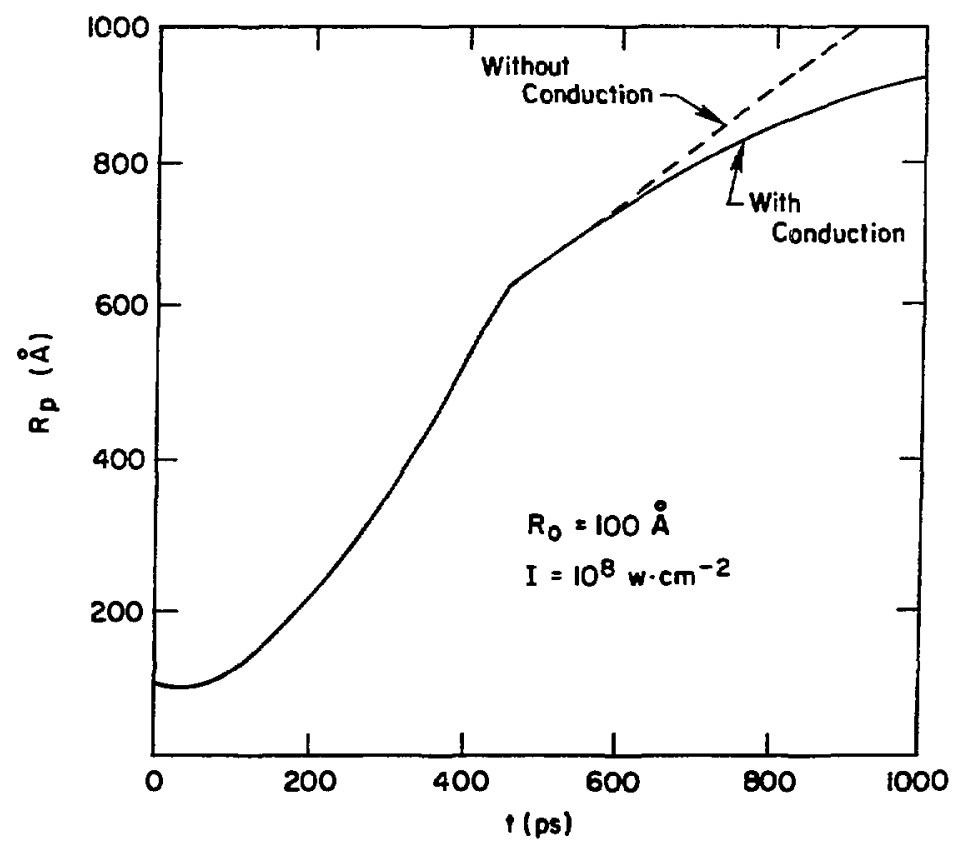

Fig. G-1.

Numerical calculation of the radius of an exploding sphere of material containing $\mathrm{C}, \mathrm{H}, \mathrm{O}$, and $\mathrm{N}$ imbedded in a medium of the same density and composition. Material has a numerically calculated equation-of-state and thermal conductivity. Solid line is with conductivity turned on and dashed line is with conductivity turned off. 


\section{FAST ELECTRON TRANSPORT}

In general, absorption of an x-ray photon by an atom will result in the emission of one or two fast electrons. For example, the fluorescent yield of nitrogen for $h v=400 \mathrm{eV}$ is less than $\frac{2}{2} \% .{ }^{54}$ The rest of the energy goes into Auger transitions which most typically will result in an electron of about $350 \mathrm{eV}$. The range of this electron may be substantial compared to the dimensions of the absorbing protein globule.

At nonrelativistic energies, the rate of energy loss ( $\mathrm{dE} / \mathrm{dx}$ ) will be approximately inversely proportional to the electron energy, and at relativistic energies the rate of energy loss will be approximately constant. In general we will be dealing with nonrelativistic electrons, and their range is well characterized by "pr" $\left(\mathrm{gm} \cdot \mathrm{cm}^{-2}\right)$ which increases roughly as the square of the initial energy .

\section{A. Early Time}

In the early stages of expansion, when the density of the protein is still about the same as the density of the cytoplasm, the early-time solutions given in Appendix $D$ are reasonably valid only we must consider the heated sphere to be of larger radius than the protein glubule itself. Clearly the heat deposition will not drop off sharply at the edges of a sphere, and the real problem should be considered numerically. Also, $\frac{\mathrm{dE}}{\mathrm{dx}}$ in the protein will differ from $\frac{\mathrm{dE}}{\mathrm{dx}}$ in the cytoplasm. At $t=0$ the effective radius of the heated sphere could be crudely approximated by

$$
\mathrm{R}_{\mathrm{eo}} \cong \frac{\eta}{\rho_{\mathrm{o}}}+\mathrm{R}_{\mathrm{o}}
$$

where $\eta$ is some constant near unity times the range of the hot electrons. Now the expansion of the protein within the sphere of radius $R_{e}$ should be nearly self similar, so its radial displacement should scale as $\left(R_{0} / R_{e o}\right)$. Furthermore the energy density within the effective sphere should scale as $\left(R_{0} / R_{e o}\right)^{3}$. Therefore Eq. (D-25) could be replaced by 


$$
R_{p}=R_{0}+\left[F(\gamma, \eta) K \Sigma_{a b s} I\right]^{1 / 2} t^{3 / 2}
$$

where

$$
F(\gamma, \eta)=\frac{2(\gamma-1)}{3(\gamma+1)^{2}(1+\xi)^{5}}
$$

and $\quad \xi=\frac{\eta}{\rho_{0} R_{0}}$

Equation (G-3) turns into Eq. (D-25) as $\eta \rightarrow 0$ because $F(\gamma, 0)=f(\gamma)$. Equation $(G-3)$ loses accuracy for $\eta>0$ after $R_{p}$ exceeds $R_{0}$ by a substantial fraction, because the assumption that the material inside the effective radius $\left(R_{e}\right)$ is of the same density as that outside breaks down.

\section{B. Late Time}

At late times the energy of the fast electrons will deposit primarily in the snowplow region, because the density within the effective radius becomes very small. When this happens, the shock front becomes a detonation front. When the shockwave has propagated far enough for almost all the fast electron energy to be deposited at the discontinuity, the radius of the detonation front will be

$$
R_{d}=\left[\hat{G}(\gamma) K \Sigma_{a b s} I\right]^{1 / 5}(R \quad t)^{3 / 5}
$$

where

$$
\hat{G}(\gamma)=\frac{250}{81}\left(\gamma^{2}-1\right)
$$

This is strictly true only if the detonation propagates at the Chapman-Jougnet point, 55,56 i.e., the flow behind the shock is sonic in shock-fixed coordinates. 
Wilson and Turcotte 57 observed that an analogous situation, a detonation front driven by a spherically converging laser beam, was in fact an overdriver detonation. But it was not so strongly overdriven as to make Eq. (F-5) a bad approximation. For example, $\hat{G}^{1 / 5}(\gamma)=1.406$, and the corresponding value that Wilson and Turcotte found by numerical integration as 1.421 , about $1 \%$ greater and hardly worth worrying about. Furthermore, because there is always some electron energy lost in transit to the detonation front Eq. (F-4) is generally a slight overestimate. The detonation front will run ahead of the shock front position given by Eq. (C-33) by a factor of

$$
\frac{R_{d}}{R}=\left[\frac{40}{81} \frac{8 \gamma-1}{\gamma+1}\right]^{1 / 5}
$$

of about 12 to $18 \%$ for practical values of $\gamma$.

While the detonation front given by $\mathrm{Eq}$. (F-4) runs ahead of the shock front given by $\mathrm{Eq}$. (D-33), the protein globule radius will lag behind that given in Eq. (D-34). After the detonation is set up, it is a reasonable approximation to say

$$
\frac{R_{d}}{R_{p}}=\text { constant }
$$

although in fact the fluid velocity downstream of the detonation fronts does not drop off linearly to zero at the origin. 57

The problem then reduces to finding the ratio of $R_{d}$ to $R_{p}$ at the time the shockwave assumes a detonation character. This can be done in a rather straightforward manner.

First we observe that the sphere of radius $R_{e}$ should maintain a constant " $\rho r "$ as its radius increases. We find that the mass inside the effective sphere is given by 


$$
m_{e}=m_{e o} \frac{R_{e}^{2}}{R_{e 0}^{2}}
$$

where

$$
\mathrm{m}_{\mathrm{eo}}=\frac{4 \pi}{3} \rho_{\mathrm{o}} \mathrm{R}_{\mathrm{eo}}^{3}
$$

Now if we assume the density within $R_{p}$ to be the same as between $R_{p}$ and $R_{e}$, then

$$
\frac{\mathrm{R}_{\mathrm{p}}^{3}}{\mathrm{R}_{\mathrm{e}}^{3}}=\frac{\mathrm{m}_{\mathrm{p}}}{\mathrm{m}_{\mathrm{e}}}
$$

where

$$
m_{p}=\frac{4 \pi}{3} \rho_{o} R_{o}^{3}
$$

Substituting Eqs. $(G-7),(G-8)$, and $(G-10)$ into Eq. (G-9) and solving for $R_{p}$, we find

$$
R_{p}=R_{o}\left[\frac{R_{e}}{R_{e o}}\right]^{1 / 3}
$$

Equation (F-11) shows how $R_{p}$ must vary with $R_{e}$ in order to maintain constant "pr" within $\mathrm{R}_{\mathrm{e}}$.

Now the mass of cytoplasm displaced by $R_{e}$ is

$$
\frac{4 \pi}{3} \rho_{o} R_{e}^{3}-\left(m_{e}-m_{e o}\right)
$$


The expanding sphere $R_{e}$ compresses the cytoplasm ahead of it like a snowplow. The thickness of the snowplow region, $\Delta \mathrm{r}$, can be found by observing that

$$
4 \pi R_{e}^{2} \Delta r \rho_{s}=\frac{4 \pi}{3} \rho_{0} R_{e}^{3}-\left(m_{e}-m_{e o}\right)
$$

where $\rho_{s}$ is the density of the snowplow region. Substituting Eqs. (G-7) and (G-8) into Eq. (G-12), we can find the "pr" of the snowplow region,

$$
\rho_{s} \Delta r=\frac{\rho_{0}}{R^{2}} R_{e}^{3}-R_{e o}^{3} \frac{R_{e}^{2}}{R_{e o}^{2}}-1
$$

Now the transition to a detonation must occur about when

$$
\rho_{s} \Delta r \cong \rho_{0} R_{e o}
$$

At this point the stopping power of the snowplow region is equal to the stopping power of the protein and cytoplasm in the sphere within the snowplow region. Thereafter, the stopping power of the snowplow region will always dominate. Substituting Eq. (G-14) into Eq. (G-13) we obtain a cubic equation for $R_{e}$ in terms of $\mathrm{R}_{\mathrm{eo}}$,

$$
R_{e}^{3}-2 R_{e o} R^{2}+R_{e o}^{3}=0
$$

Notice that we do not have to know the density of the snowplow region explicitly. The nontrivial solution to Eq. (G-15) gives the effective radius at transition to a detonation, 


$$
\mathrm{R}_{\mathrm{e}}^{\mathrm{T}} \cong \mathrm{q} \mathrm{R}_{\text {eo }}
$$

where

$$
\mathrm{q} \cong 1.6180
$$

Equation (G-11) gives the protein radius at transition to a detonation, and using Eq. (G-2) we can evaluate the constant in Eq. (G-6)

$$
\begin{aligned}
\frac{R_{d}}{R_{p}} \cong \frac{R_{e}^{T}}{R_{p}^{T}} \\
=\frac{q R_{e o}}{q^{1 / 3} R_{o}} \\
=q^{2 / 3}(1+\zeta) .
\end{aligned}
$$

So,

$$
R_{p} \cong \frac{q^{-2 / 3} R_{d}}{1+\xi}
$$

Now if $\eta \ll \rho_{o} R_{o}, R_{P} \cong q^{-2 / 3} R_{d}=0.7256 R_{d}$, or for $\gamma=5 / 3, R_{p} \cong 0.8859 R$ where $R$ is given by Eq. (D-33). However, $R_{p} \cong 0.9086 R$, when $R_{p}$ is given by Eq. (D-34). These are remarkably similar, but we would really like Eq. (F-15) to converge identically to Eq. $(D-34)$ as $\eta \rightarrow 0$.

Figure G-2 compares the function $g^{1 / 5}(\gamma)$ given by Eq. (D-34) with the function $\mathrm{q}^{-2 / 3 \mathrm{G}^{1 / 5}}(\gamma)$ as defined by Eqs. $(\mathrm{G}-4)$ and $(\mathrm{G}-16)$ over the range of $\gamma$ in which we have interest. It shows the actual difference in radius of the protein as $\eta \rightarrow 0$ given by Eq. $(D-34)$ and Eq. (G-15) as a function of $\gamma$. The behavior of 


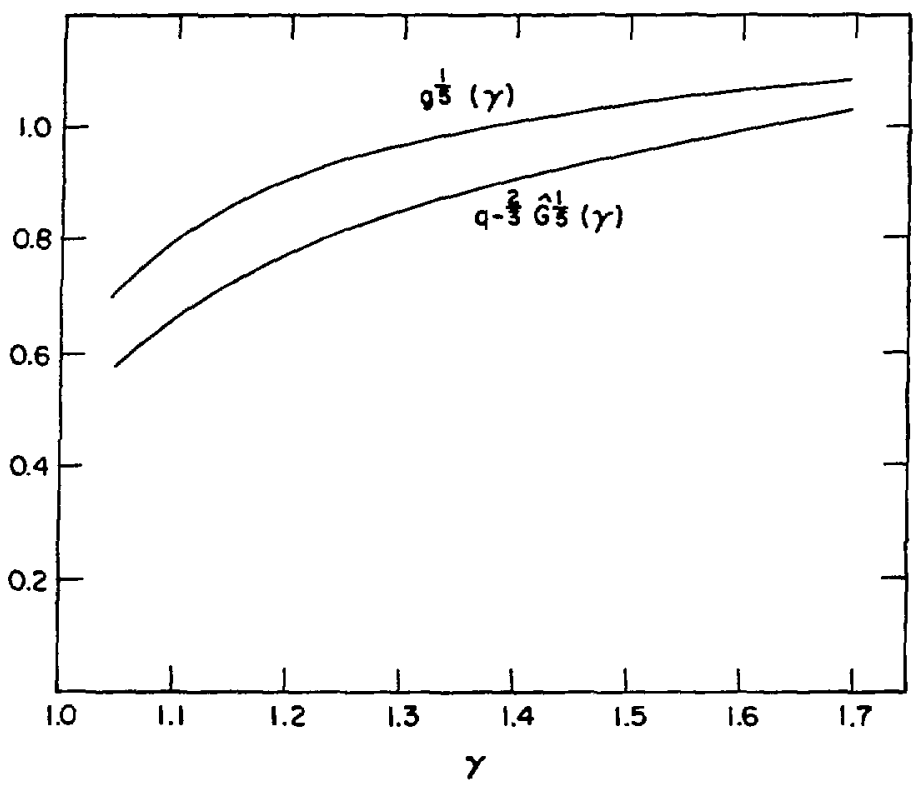

Fig. G-2.

Comparison of $\mathrm{g}^{1 / 5}(\gamma)$ with $\mathrm{q}^{2 / 3} \mathrm{G}^{\mathrm{i}} \mathrm{s}(\gamma)$ as a function of $\gamma$.

the functions is quite similar, their difference ranging from $5 \%$ at $\gamma=1.7$ to $21 \%$ at $y=1.05$. They could be even more similar had we chosen a less conservative definition for the transition from a shockwave to a detonation. It is therefore reasonable to approximate the radius of the protein with

$$
R_{P}=\left[G(\gamma, \eta) K \Sigma_{a b s} I\right]^{1 / 5}\left(R_{0} t\right)^{3 / 5}
$$

where

$$
\begin{aligned}
G(\gamma, \eta) & =\frac{\xi q^{-10 / 3} \hat{G}(\gamma)}{(1+\zeta)^{5}}+\frac{g(\gamma)}{(1+\zeta)} \\
& =\left\{\frac{\xi q^{-10 / 3}}{(1+\xi)^{5}}\left[\frac{40}{81} \frac{8 \gamma-1}{\gamma+1}\right]+\left(\frac{2}{\gamma+1}\right)^{5 / 3} \frac{\hat{g}(\gamma)}{1+\zeta}\right\}
\end{aligned}
$$




$$
\zeta=\frac{\eta}{\rho_{0} R_{0}}
$$

This expression preserves the property that $G(\gamma, 0)=g(\gamma)$, and goes to a purely detonation expression when $\eta \gg \rho_{0} R_{0}$. 


\section{APPENDIX $\mathrm{H}$}

\section{GLOSSARY OF SYMBOLS}

(English and Greek letters have the following meanings unless locally defined otherwise.)

I. LOWER CASE ENGLISH

a = characteristic transverse dimension of specimen

b = distance from specimen to recording surface

$c \quad=$ speed of light

$\mathrm{d}=$ characteristic transverse dimension of opaque structure within specimen

e = electronic charge

= distance from center of hologram on planar recording surface

$$
\begin{aligned}
& f(\gamma)=\frac{2}{3} \frac{(\gamma-1)}{(\gamma+1)^{2}} \\
& \hat{f}(\gamma)=\frac{(\gamma-1)}{6} \\
& g(\gamma)=\left(\frac{2}{\gamma+1}\right)^{5 / 3} \frac{25}{4} \frac{(\gamma+1)\left(\gamma^{2}-1\right)}{(8 \gamma-1)} \\
& \hat{g}(\gamma)=\frac{25}{4} \frac{(\gamma+1)\left(\gamma^{2}-1\right)}{(8 \gamma-1)} \\
& \mathrm{h} \quad=\text { Planck's constant }^{\prime} \\
& i=\text { specific energy for free } r \text { run expansions } \\
& \mathrm{k}=\text { Boltzman constant } \\
& \ell=\text { distance scattered photon must travel from specimen to recording surface } \\
& \mathbf{m}=\text { mass } \\
& m_{p}=\text { mass of protein } \\
& m_{e}=\text { mass of effective sphere including heat transport } \\
& m_{e 0}=\text { initial value of } m_{e}
\end{aligned}
$$


$\mathrm{n}=$ noise from all sources other than incoherent interference

$r \quad=$ radius

$\mathrm{q}=\mathrm{R}_{\mathrm{e}}^{\mathrm{T}} / \mathrm{R}_{\mathrm{eO}}$

$r_{1}, r_{2}=$ radii of concentric shells

$s=\sigma T^{4}=$ radiant energy flux from the surface of a perfect black body

$t=$ time

$\bar{v} \quad=$ thermal average atomic velocity

$v_{t}, v_{r}=$ tangential and radial electron velocities

$\mathrm{x}=\mathrm{x}$-ray temporal coherence length

$y=x$-ray spatial coherence length

II. UPPER CASE ENGLISH

A = atomic weight

$C_{p}=$ specific heat at constant pressure

$C_{v}=$ specific heat at constant volume

$E \quad=$ total energy

$\mathbf{E}_{0}=$ initial energy

$F(\gamma, \eta)=\frac{2(\gamma-1)}{3(\gamma+1)^{2}(1+\zeta)^{5}}$

$G(\gamma, \eta)=\frac{\xi q^{-103 \hat{G}(\gamma)}}{(1+\zeta)^{5}} \frac{g(\gamma)}{(1+\zeta)}$ heat transport parameters

$I \quad=x$-ray intensity

$\mathrm{K}=$ number of protein/nucleic acid units per gram if specimen

$\mathrm{L}$ = angular momentum

$M \quad=$ mass of a snowplow region

$\mathrm{N}$ = noise from incoherent interference

$P_{p}=$ pressure inside an exploding protein globule 


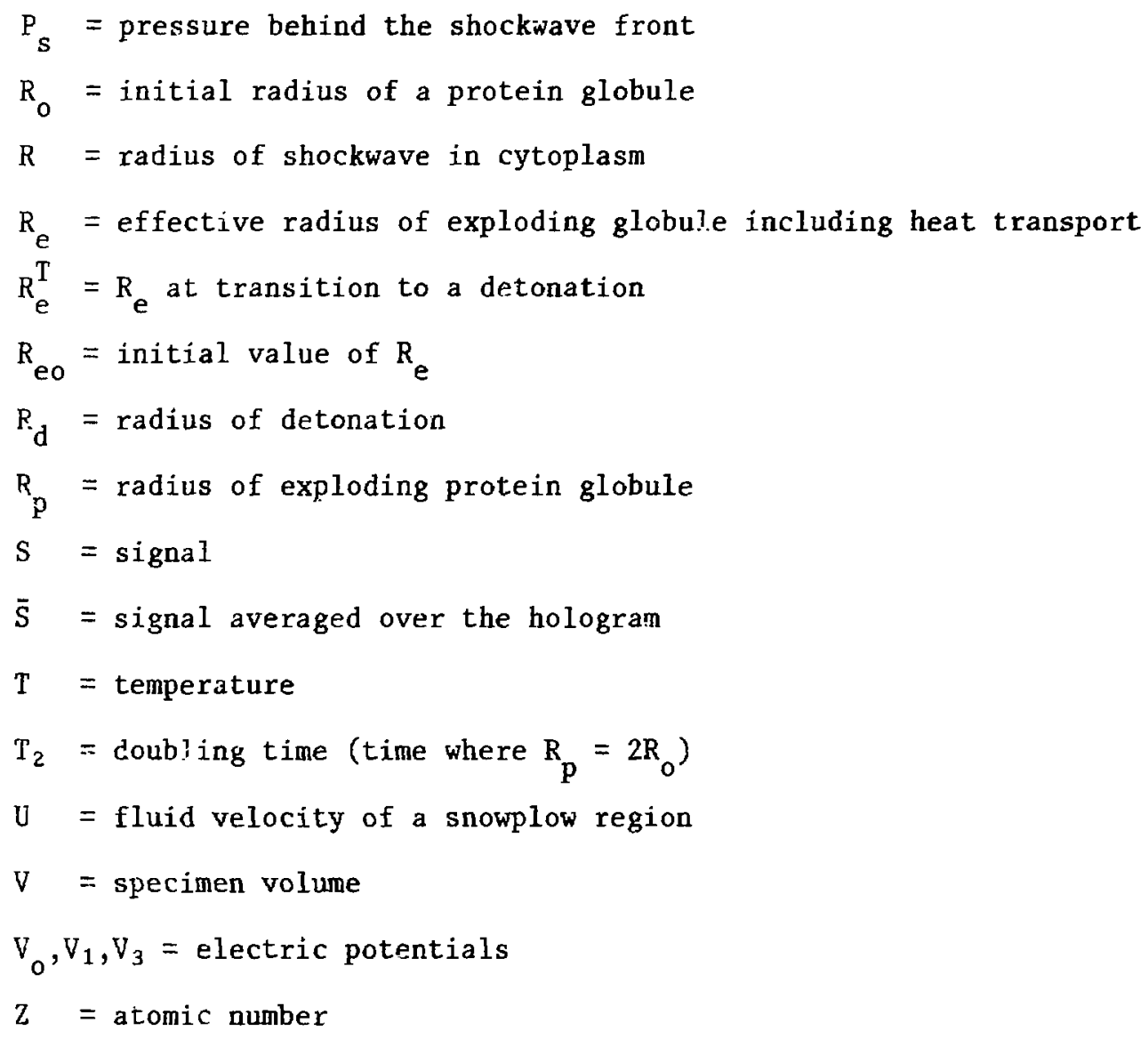




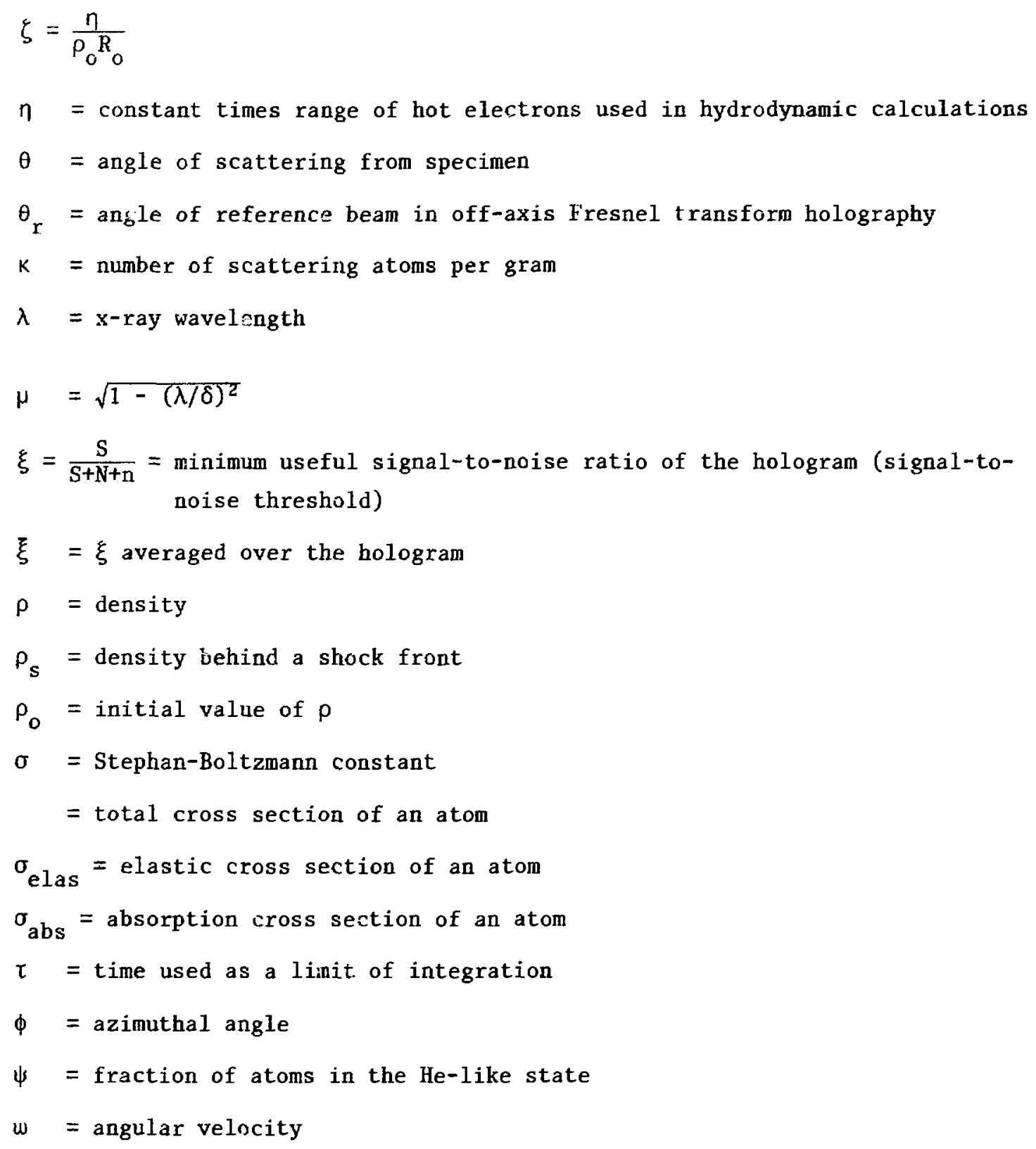


IV. UPPER LASE GREEK

$\Gamma_{\text {nat }}=$ natural linewidth

$\Gamma_{\text {res }}=$ resonant linewidth

$\Delta=$ linear resolution, referred to recording medium (e.g., grain size)

$\Delta \mathrm{r}=$ increment of radius

$\Delta t=$ optimum exposure time

$\Delta \phi \quad=$ phase blurring

$\Sigma=$ total cross section of a protein/nucleic acid unit

$\Sigma_{a b s}=$ elastic cross section of a protein/nucleic acid unit

$\Sigma_{\text {elas }}=$ absorption cross section of a protein/nucleic acid unit

$\Sigma_{1}=$ total cross section for atomic coherent scattering

$\Sigma_{2}=$ total cross section for shadow coherent scattering

ACKNOWLEDGMENTS

I wish to acknowledge $G$. Chapline for his active participation and substantial contributions; G. Baldwin for his thoughts and contributions; J. Kirz for sharing his insight and wisdom in the field of x-ray microscopy; A. Petschek for his quixotic efforts to keep us honest; F. Harlow and H. Ruppe 1 for the numerical hydrodynamic calculations; G. Bel1, J. Costa, M. Dembo, and M. Bitensky for their interest, suggestions, and biological pedigogy; and a myriad of other for their participation and helpful criticism. 


\section{REFERENCES}

1. K. Porter and J. Tucker, Sci. American 244, 57 (1981).

2. J. Goodman and R. Lawrence, App1. Phys. Lett. 11, 77 (1967).

3. P. Lewis and D. Knight, Staining Methods for Sectioned Material (North Holland Publishing Co., Amsterdam-New York-Oxford, 1977).

4. H. Piller, Microscope Photometry (Springer-Verlag, Berlin-Heidelberg, New York, 1977).

5. J. Kirz and D. Sayre, "Soft X-Ray Miscrscopy of Biological Specimens," in Synchrotron Radiation Research (Plenum Press, New York-London, 1980), pp. 277-322.

6. J. Costa, National Institute of Health, private communication, April 1982.

7. M. Bitensky, Los Alamos National Laboratory, private communication, October 1981. Also see M. Bitensky, G. Wheeler, A. Yamazaki, M. Rasenick, and J. Stein, "Cyclic-Nucleotide Metabolism in Vertebrate Photoreceptors," in Current Topics in Membranes and Transport, 15 (Academic Press Inc., New York, 1981), pp. 237-271.

8. G. Bell, Los Alamos National Laboratory, private communication, November 1981 .

9. M. Dembo, Los Alamos National Laboratory, private communication, March 1982 .

10. D. Gabor, Nature 161, 777 (1948).

11. D. Gabor, Proc. Roy. Soc. A197, 454 (1949).

12. A. Baez, J. Opt. Soc. Am. 42, 756-762 (1952).

13. A. Baez and H. E1-Sum, X-Ray Microscopy and Microradiography (Academic Press, Inc., New York, 1957), pp. 347-366.

14. G. Stroke and D. Falconer, J. Opt. Soc. Am. 55, 595 (1965).

15. G. Stroke and R. Restrick, App1. Phys. Let. I, 229-231 (1966).

16. G. Stroke, "Attainment of High Resolution," in Optique des Rayons X et Microanalyse (Hermann, Paris, 1966), p. 43.

17. G. Rogers and J. Palmer, J. Microsc. 89, 125-134 (1969).

18. J. Breedlove and S. Trammell, Science 170, 3964, pp. 1310-12 (1970).

19. S. Aoki, Y. Ichihara, and S. Kikuta, Jpn. J. Appl. Phys. 11, 1857 (1972). 
20. S. Aoki and S. Kikuta, Jpn. J. Appl. Phys. 13, 1385-1392 (1974).

21. B. Reuter and H. Mahr, J. Phys. E. 9, 746-751 (1976).

22. G. Bjorklund, Stanford Microwave Laboratory report No. 2339 (1974).

23. G. Bjorklund, S. Harris, and J. Young, Appl. Phys. Lett. 25, 451 (1974).

24. G. Chapline and L. Wood, Phys. Today 28, 40 (1975).

25. A. Kondratenko and A. Skrinsky, Optical Information Processing, Vol. 2, (Plenum Press). p-1.

26. R. Mueller and S. Jorna, App1. Opt. 16, 525-526 (1977).

27. A. Giese, Cell Physiology (Saunders Co., Philadelphia-London-Toronto, 1973), p. 53 .

28. A. L. Lehninger, Biochemistry (Worth Publishers, Inc., New York, 1975).

29. De Robertis and De Robertis, Cell and Molecular Biology (Saunders Co., Philadelphia-London-Toronto, 1980).

30. J. De Velis, G. Parrent, Jr., and B. Thompson, J. Opt. Soc. Am. 56, 423 (1966).

31. E. Leiter and J. Upatnieks, J. Opt. Soc. Am. 53, 1377 (1963).

32. R. Feder, E. Spiller, and J. Topalian, Polymer Eng. \& Sci. 17, 385-389 (1977).

33. R. Feder, E. Spiller, J. Topalian, A. Broers, W. Gudat, B. Panessa, Z. Zadunaisky, and J. Sedat, Science 197, 259-260 (1977).

34. R. Prohaska and A. Fisher, App1. Phys. Lett. 40(3), 283-285 (1982).

35. F. Pollack and S. Lowentha1, Rev. Sci. Instrum. 52, 207-212 (1981).

36. L. Murr, Electron Optical Applications in Materials Science (McGraw-Hill, Inc., New York, 1970), p. 95.

37. A zone plate whose finest spacing is $800 \AA$ is being used in an $x$-ray microscope by J. Kirz, University of New York, Stony Brook (private communication, October 1981). Zone plates with finer spacing are under development.

38. This method has been considered by J. Kare, Lawrence Livermore National Laboratory in an unpublished document on $x$-ray holography.

39. J. Fries, Los Alamos National Laboratory, private communication, October 1981 .

40. R. Benjamin, P. Lyons, and R. Day, Appl. Opt. 16, 393-397 (1977).

41. G. Farnel1, J. Phot. Sci. 17, 116-125 (1969). 
42. B. Henke, J. Knauer, and K. Premaratne, J. App1. Phys. 52, 1509-1520 (1981).

43. G. McCall, Los Alamos National Laboratory, private communication, July 1982.

44. F. Harlow and A. Amsden, "Fluid Dynamics," Los Alamos Scientific Laboratory report LA-4700 (1971).

45. J. Jackson, Classical Electrodynamics (John Wiley \& Sons, Inc., New YorkLondon, 1962), pp. 299-304.

46. S. Wallace, Annals of Physics, 78, 190-257 (1973).

47. B. Henke, Advances in X-Ray Analysis, Vol. 7 (Plenum Press, New York, 1965), pp. 460-48

48. E. Spiller, "Evaporated Multilayer Dispersion Elements for Soft X-Rays, Low Energy X-Ray Diagnostics, AIP, New York, pp. 122-145.

49. V. Weisskopf and E. Wigner, Zs. F. Phys. 63, 54 (1930; 65, 18 (1930).

50. J. Schulman and J. Dow, Phys. Rev. Lett., 47, 371-374 (1981).

51. A. Bianconi, H. Petersen, F. Brown, and R. Bachrach, Phys. Rev. A 17, 19071911 (1978).

52. G. King, F. Read, and M. Trone, Chem. Phys. Lett. 52, 50-53 (1977).

53. This derivation follows roughly the treatment of strong explosions in a homogeneous atmosphere given by Ya. Zel'dovich and Y. Raizer, Physics of Shock Waves and High-Temperature Hydrodynamic Phenomena, Academic Press (1966), pp. 93-99; also see G. Chernyi, Dok1. Akad. Nauk SSSR 112, 213-216 (1957).

54. R. Fink, R. Jopson, H. Mark, and C. Swift, Atomic Fluorescence Yields, UCRL-14327, University of California (1965).

55. Ya. Zel'dovich, Zh. Eksperim. i Teor. Fix. 10, 542-568 (1940).

56. Ya. Zel'dovich and A. Kompaneets, Theory of Detonation (Academic Press, New York, 1960).

57. C. Wilson and D. Turcotte, J. Fluid Mech. 43, 399-406 (1970). 


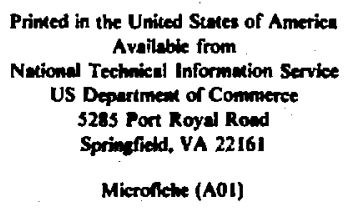

NTIS

Page Range Price Code

001.025

$026-050$

$051-075$

076-100

101-125

$126-150$

\section{$\mathrm{A02}$}

A03

A04

AOS

A06
NTtS

\begin{tabular}{cc} 
Page Range & Price Code \\
\hline $151-175$ & AOs \\
$176-200$ & $A 09$ \\
$201-225$ & $A 10$ \\
$226-250$ & $A 11$ \\
$251-275$ & $A 12$ \\
$276-300$ & $A 13$
\end{tabular}

NTtS

Pege Racere Price Code

326-330

351-375

$376-400$

401-425

$426-450$
A1s

A16

A17

A18

AIs
NTIS

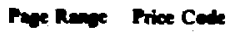

451-475

5a1-525 A22

$526.530 \quad A 23$

$551-575 \quad A 24$

576-0 A25

-Conact NTIS for a price quote. 
